Florida International University

FIU Digital Commons

6-17-2014

\title{
The Effects of Advanced Practice Nurses (APNs) as Intensivists in a Surgical Intensive Care Unit (SICU) on Patient Outcomes, Healthcare Charges, and APN Intensivist Services in the SICU
}

Francisco Guido-Sanz

Florida International University, fgsrn@msn.com

DOI: $10.25148 /$ etd.FI14071169

Follow this and additional works at: https://digitalcommons.fiu.edu/etd

\section{Recommended Citation}

Guido-Sanz, Francisco, "The Effects of Advanced Practice Nurses (APNs) as Intensivists in a Surgical Intensive Care Unit (SICU) on Patient Outcomes, Healthcare Charges, and APN Intensivist Services in the SICU" (2014). FIU Electronic Theses and Dissertations. 1544.

https://digitalcommons.fiu.edu/etd/1544

This work is brought to you for free and open access by the University Graduate School at FIU Digital Commons. It has been accepted for inclusion in FIU Electronic Theses and Dissertations by an authorized administrator of FIU Digital Commons. For more information, please contact dcc@fiu.edu. 


\title{
FLORIDA INTERNATIONAL UNIVERSITY \\ Miami, Florida
}

\section{THE EFFECTS OF ADVANCED PRACTICE NURSES (APNs) AS INTENSIVISTS IN} A SURGICAL INTENSIVE CARE UNIT (SICU) ON PATIENT OUTCOMES, HEALTHCARE CHARGES, AND APN INTENSIVIST SERVICES IN THE SICU

\author{
A dissertation submitted in partial fulfillment of \\ the requirements for the degree of \\ DOCTOR OF PHILOSOPHY \\ in \\ NURSING \\ by \\ Francisco Guido-Sanz
}




\section{To: Dean Ora Lea Strickland}

Nicole Wertheim College of Nursing and Health Sciences

This dissertation, written by Francisco Guido-Sanz, and entitled The Effect of Advanced Practice Nurses (APNs) as Intensivists in a Surgical Intensive Care Unit (SICU) on Patient Outcomes, Healthcare Charges, and APN Intensivist Services in the SICU, having been approved in respect to style and intellectual content, is referred to you for judgment.

We have read this dissertation and recommend that it be approved.

JoAnne M. Youngblut

Ellen L. Brown

Timothy Page

Dorothy Brooten, Major Professor

Date of Defense: June 17, 2014

The dissertation of Francisco Guido-Sanz is approved.

Dean Ora Lea Strickland Nicole Wertheim College of Nursing and Health Sciences

Dean Lakshmi N. Reddi

University Graduate School

Florida International University, 2014 
(C) Copyright 2014 by Francisco Guido-Sanz

All rights reserved. 


\section{DEDICATION}

I dedicate this dissertation to my loving parents whom have been an endless and sound source of love, support, and understanding. Pipo and Mima, how I endearingly call them, you are the source of inspiration that drives me to tenaciously strive to pursue and achieve my dreams and goals in life.

To Peter, my loyal, caring, and unwavering partner, thank you for your unconditional support and understanding and for enduring all these years of a neverending academic journey. Thank you for being an indispensable part of my life.

To my beloved sons Guelmi and Kenny, thank you for all your love and support, and let this work serve you as a reminder to pursue your dreams with patience and perseverance; you will triumph over any obstacle.

My brother Jesus, and nephews Alejandro and Isabella, you are also part of my success and thus I dedicate this work to you also. Thank you for your unyielding love.

To my godfather Roberto Martinez, thank you for your faith in me and for all your guidance and advice.

To my lifelong friends Michael Roe, Amalia Santiago, and Analid Diaz, thank you for your steadfast friendship, for your resolute love, and for all your steady support throughout time.

To my friend and colleague Suha, for your support was instrumental in this trajectory, bringing wisdom, pragmatism, and fun to this journey. Thank you for your keen friendship, help, and advice.

To all my dear friends, you know who you are, thanks for believing in me. 


\section{ACKNOWLEDGMENTS}

I would like to thank my Major Professor Dr. Dorothy Brooten for all of her guidance, support, and advice given throughout my doctoral education. Dr. Brooten has been a beacon of mentorship and instrumental in helping me achieving this professional and academic milestone. I thank her for her patience and understanding and for providing me with emotional comfort and sustenance when most needed. Thank you for encouraging me to always persevere relentlessly.

I thank my committee members Drs. Youngblut, Brown, and Page for their continuous source of advice and for helping me appraise my work from different angles and perspectives. Your guidance was instrumental in the completion of my research work and dissertation defense. Thank you for your many hours of reading, reflecting, and evaluating my work and for your patience throughout the entire process.

My timeless gratitude to Isis Zambrana, Corporate Director of Quality and Patient Safety Division at Jackson Memorial Hospital (JMH), for facilitating my access to data and abstraction with the help of Abid Sattar, Associate Director of Quality and Safety at JMH. Your support will never go unnoticed or unappreciated. You were instrumental in allowing me to complete my research work and to achieve my doctoral degree.

My thanks extend to Salma Hernandez for also facilitating access to data at JMH and to Tanya Quiroz for all her help editing and proofing the manuscript.

Lastly, I thank my peers and colleagues at JMH, and especially Dr. Andrea Archer, for her sustained friendship, guidance, encouragement, and support throughout the years we worked alongside at JMH Surgical Intensive Care Unit (SICU). 


\title{
ABSTRACT OF THE DISSERTATION
}

THE EFFECTS OF ADVANCED PRACTICE NURSES (APNs) AS INTENSIVISTS IN A SURGICAL INTENSIVE CARE UNIT (SICU) ON PATIENT OUTCOMES, HEALTHCARE CHARGES, AND APN INTENSIVIST SERVICES IN THE SICU by

\author{
Francisco Guido-Sanz
}

Florida International University, 2014

Miami, Florida

Professor Dorothy Brooten, Major Professor

Intensive Care Units (ICUs) account for over 10 percent of all US hospital beds, have over 4.4 million patient admissions yearly, approximately 360,000 deaths, and account for close to $30 \%$ of acute care hospital costs. The need for critical care services has increased due to an aging population and medical advances that extend life. The result is efforts to improve patient outcomes, optimize financial performance, and implement models of ICU care that enhance quality of care and reduce health care costs. This retrospective chart review study examined the dose effect of APN Intensivists in a surgical intensive care unit (SICU) on differences in patient outcomes, healthcare charges, SICU length of stay, charges for APN intensivist services, and frequency of APNs special initiatives when the SICU was staffed by differing levels of APN Intensivist staffing over four time periods (T1-T4) between 2009 and 2011. The sample consisted of 816 randomly selected (204 per T1-T4) patient chart data. Study findings indicated reported ventilator associated pneumonia (VAP) rates, ventilator days, catheter days and catheter associated urinary tract infection (CAUTI) rates increased at T4 (when 
there was the lowest number of APN Intensivists), and there was increased pressure ulcer incidence in first two quarters of T4. There was no statistically significant difference in post-surgical glycemic control $(\mathrm{M}=142.84, \mathrm{SD}=40.00), t(223)=1.40, p=.17$, and no statistically significant difference in the SICU length of stay among the time-periods ( $\mathrm{M}=$ 3.27, $\mathrm{SD}=3.32), t(202)=1.02, p=.31$. Charges for APN services increased over the 4 time periods from $\$ 11,268$ at $\mathrm{T} 1$ to $\$ 51,727$ at $\mathrm{T} 4$ when a system to capture APN billing was put into place. The number of new APN initiatives declined in T4 as the number of APN Intensivists declined. Study results suggest a dose effect of APN Intensivists on important patient health outcomes and on the number of APNs initiatives to prevent health complications in the SICU.

Key Words: APN Intensivists, Patient Outcomes, Healthcare Charges, APN Intensivists Initiatives, Dose Effect 


\section{TABLE OF CONTENTS}

CHAPTER

PAGE

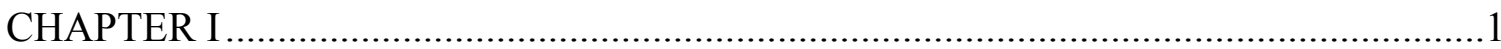

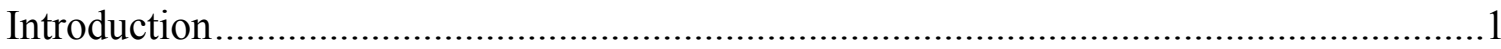

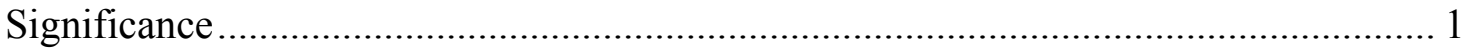

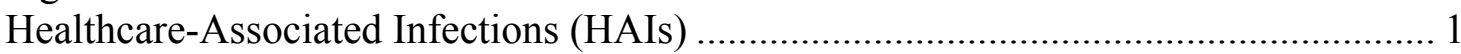

Ventilator Associated Pneumonia (VAP) ................................................................ 3

Catheter-Associated Urinary Tract Infections (CAUTI) ………............................... 4

Pressure Ulcers.......................................................................................... 5

Additional Factors Affecting ICU Length of Stay, Patient Outcomes, and Healthcare

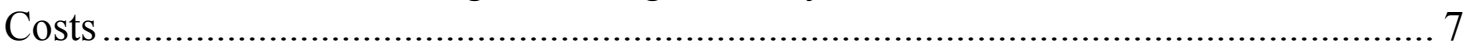

Patient-Initiated Removal of Devices.............................................................. 7

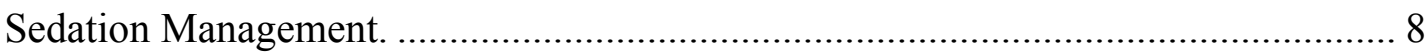

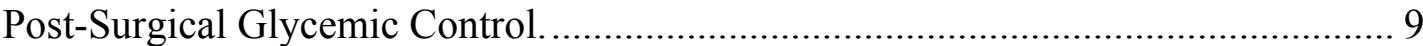

Ventilator Weaning and Ventilator Days. ................................................................ 11

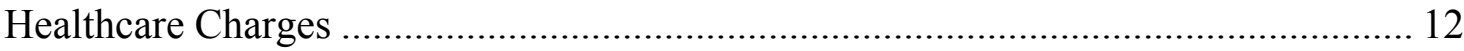

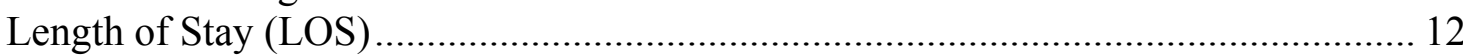

Types and Levels of ICU Staffing......................................................................... 13

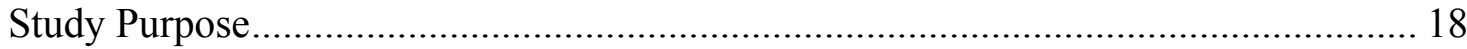

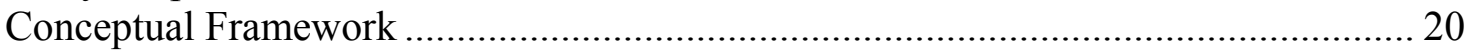

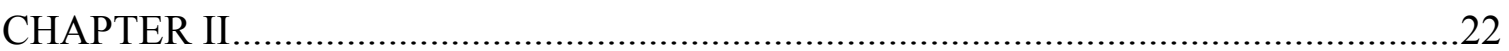

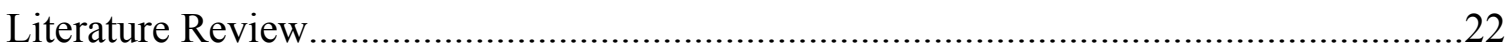

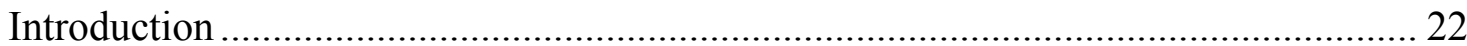

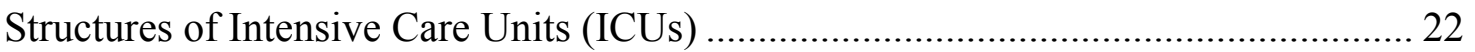

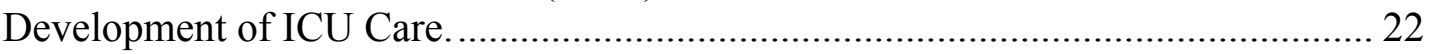

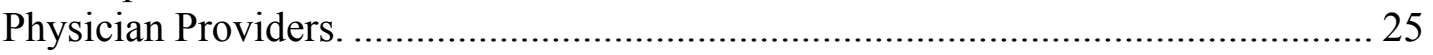

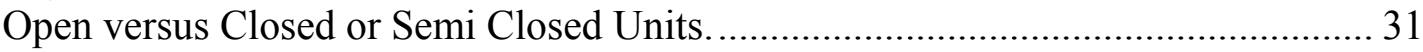

Advanced Practice Nurse (APN) Intensivist Services ................................................. 38

History of APNs in Acute Care ........................................................................... 41

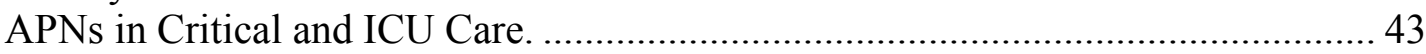

Acute Care Nurse Practitioners in Critical and ICU Care....................................... 48

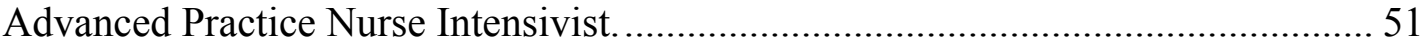

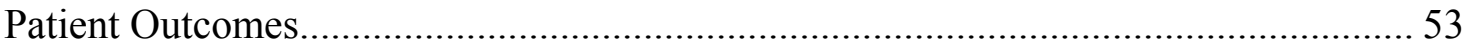

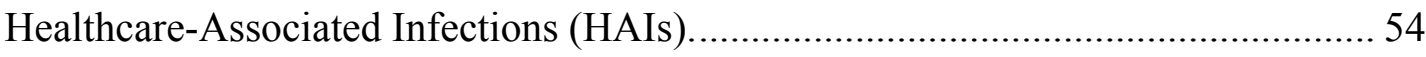

Catheter Associated Urinary Tract Infections (CAUTI) . .......................................... 73

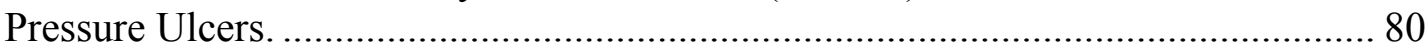

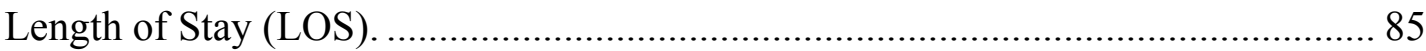

Patient Initiated Removal of Devices................................................................ 88

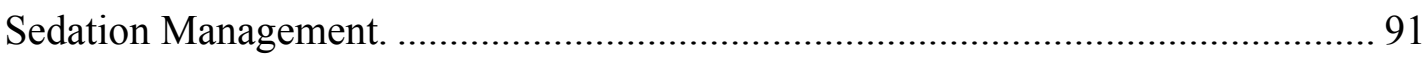

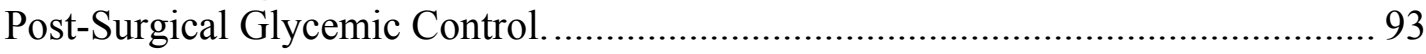

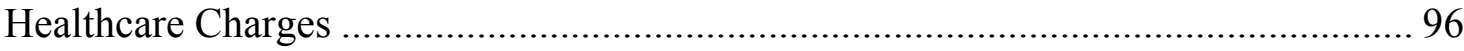

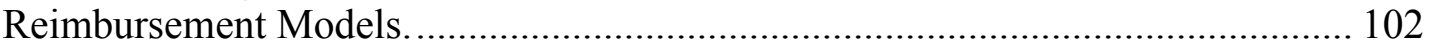




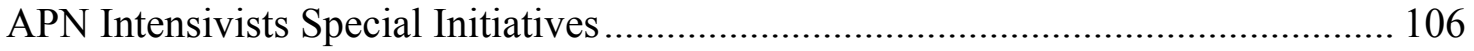

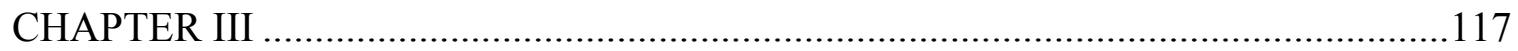

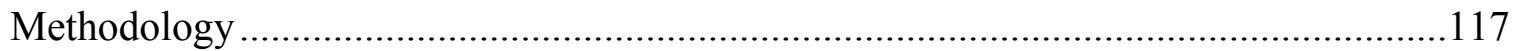

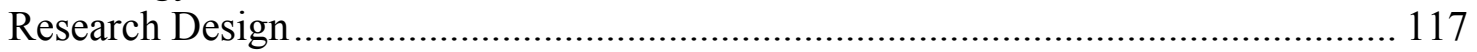

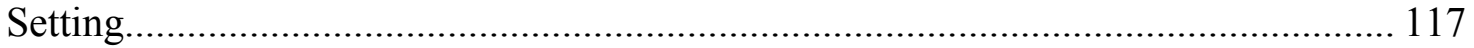

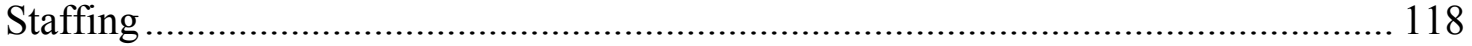

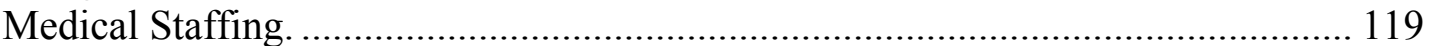

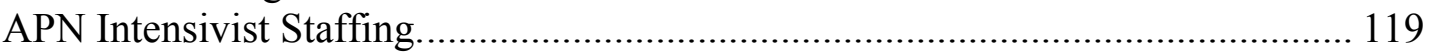

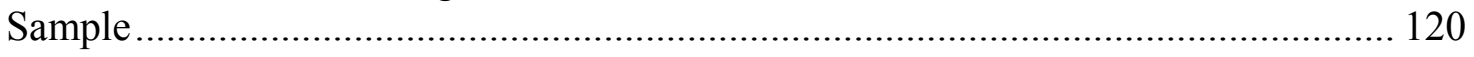

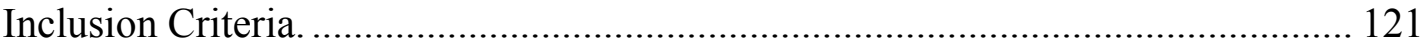

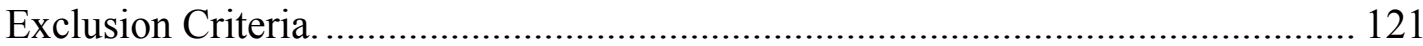

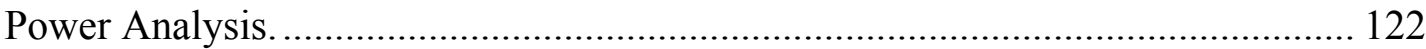

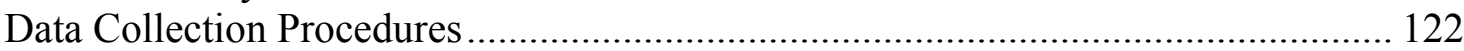

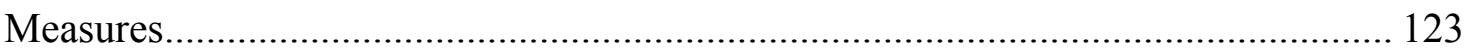

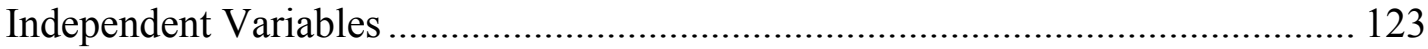

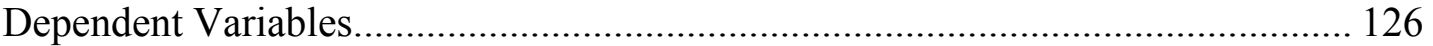

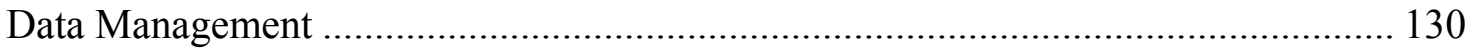

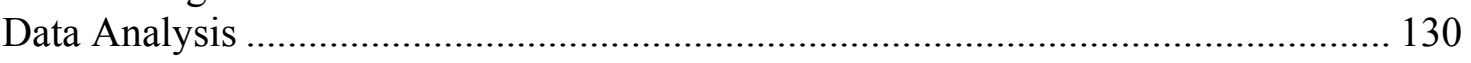

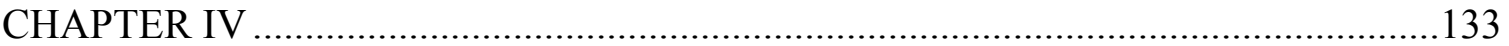

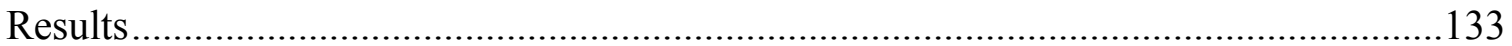

APN Intensivists, Registered Nurses, and Medical Staffing ....................................... 134

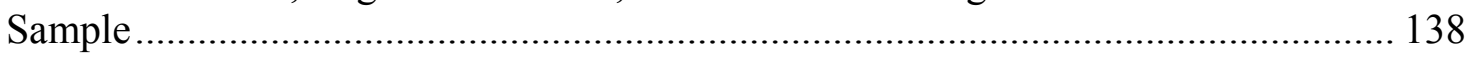

Differences in Patient Outcomes Comparing T1 versus T4; T2 versus T4; and T3

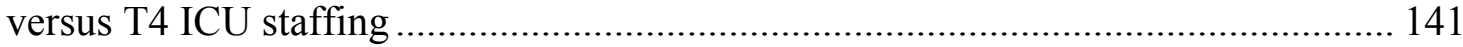

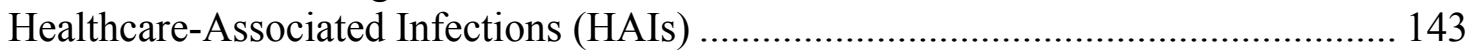

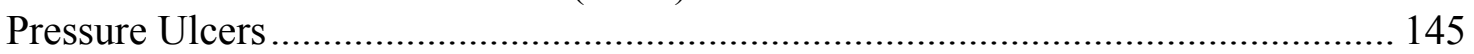

Post-Surgical Glycemic Control......................................................................... 148

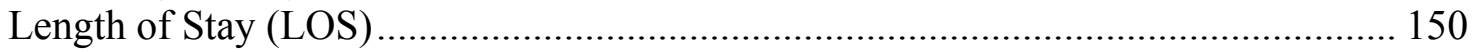

Differences in Healthcare Charges for SICU Length of Stay and APN Intensivists'

Services Comparing T1 vs. T4; T2 vs. T4; and T3 vs. T4 ICU Staffing .................... 152

Charges for APN Intensivists' Services ....................................................................... 153

Differences in the Frequency of APN Intensivist Special Initiatives Conducted Over the Four Time Periods (T1, T2, T3, and T4)...................................................... 155

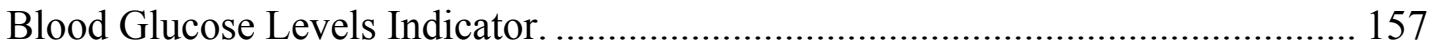

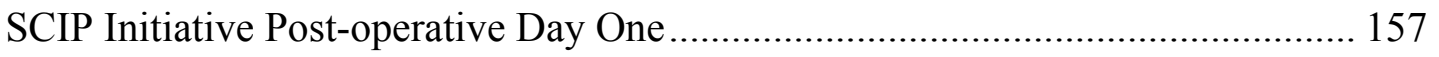

Cardiac Admission Quality Checklist. ............................................................... 158

Daily Quality Initiatives List. .............................................................................. 159

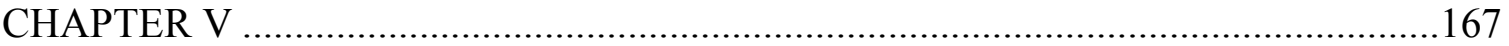

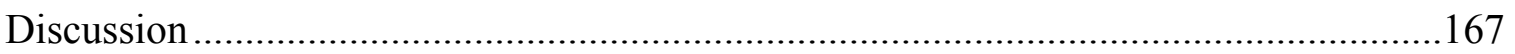

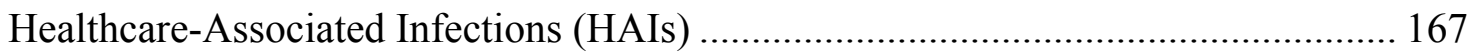

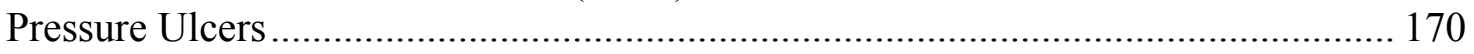

Post-Surgical Glycemic Control........................................................................ 172 
Length of Stay (LOS)

Healthcare Charges for SICU Length of Stay and APN Intensivists' Services .......... 175

Frequency of APN Intensivist Special Initiatives ……............................................ 177

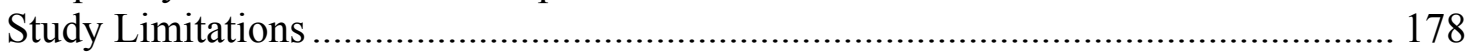

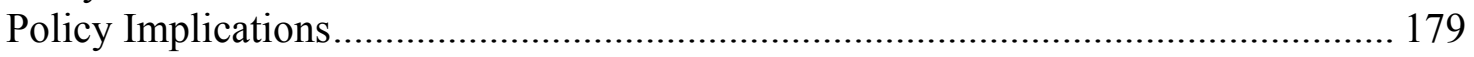

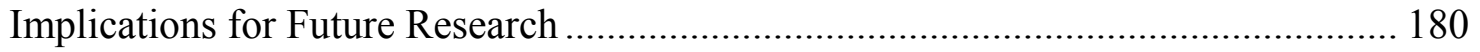

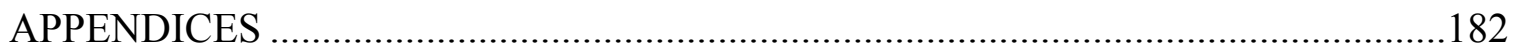

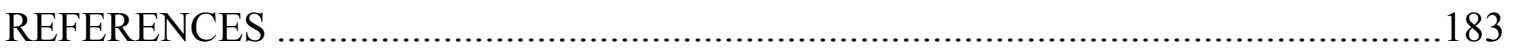

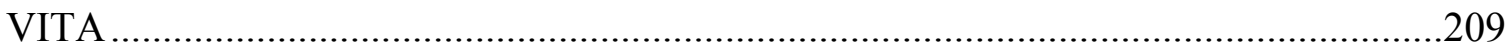




\section{LIST OF TABLES}

TABLE

PAGE

1. Study's Variables, Measures, and Data Sources .129

2. Research Questions Variables and Statistical Analysis 130

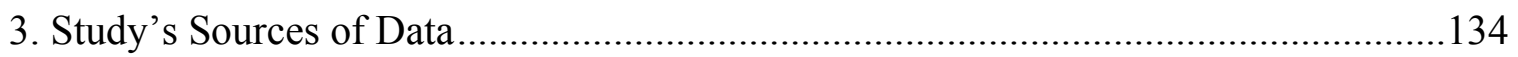

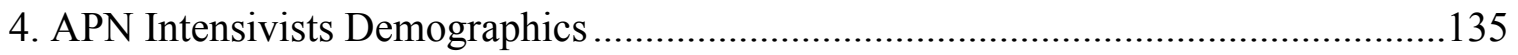

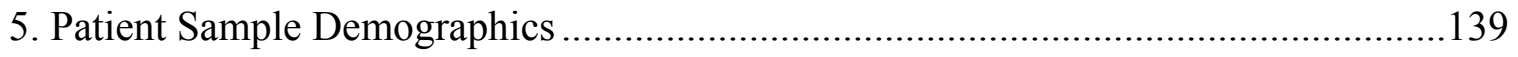

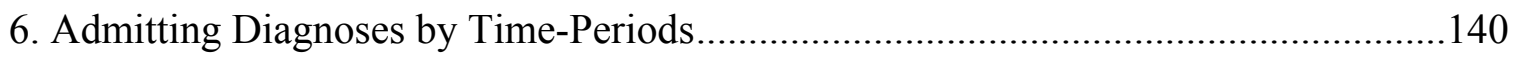

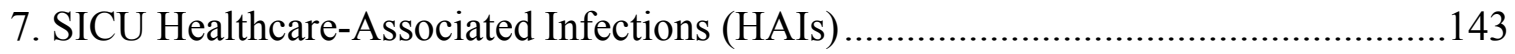

8. Pressure Ulcers in the SICU by Time-Periods (Quarters) ....................................145

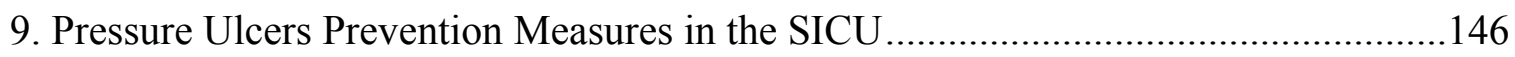

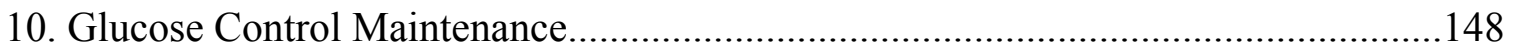

11. Glucose Fallouts Comparison Between Time Periods..........................................150

12. SICU Length of Stay by Time Periods ........................................................ 150

13. Length of Stay Comparison Between Time Periods .............................................152

14. APN Intensivists Initiatives Over Four Time-Periods (T1-T4) .............................155 


\section{CHAPTER I}

\section{Introduction}

\section{Significance}

Intensive Care Units (ICUs) account for over 10 percent of all US hospital beds, have over 4.4 million patient admissions yearly, approximately 360,000 deaths, and account for close to $30 \%$ of acute care hospital costs, (U.S. Department of Health \& Human Services, Health Resources \& Services Administration [USDHHS, HRSA], 2006). And the need for critical care services has continued to grow due to an increased aging population and medical advances that have extended life expectancy. This increased demand for ICU services has resulted in efforts to improve patient outcomes, optimize financial performance, and implement models of care in ICUs that will enhance the quality of care and reduce health care costs. Increased costs of ICU care can be attributed to the emergence of major complications including Healthcare Associated Infections (HAIs) and pressure ulcers that result in additional diagnostic procedures, extended medical management, and prolonged hospitalizations. Additional factors that may prolong ICU length of stay and affect patient outcomes and health care costs include patient-initiated accidental removal of devices, sedation management, post-surgical glycemic control, ventilator days, and ventilator weaning. Differing types and levels of staffing in ICUs can also effect patient outcomes and have wide variances in health care costs.

\section{Healthcare-Associated Infections (HAIs)}

Nearly 2 million cases of Healthcare-Associated Infections (HAIs) occur annually in hospitalized patients in the US, resulting in approximately 100,000 deaths and more 
than $\$ 17$ billion in health care costs (Jarvis, 2007; Parekh, 2008; Yokoe \& Classen, 2008). HAIs are infections associated with healthcare delivery in any setting. Patients admitted to ICUs are at even greater risk for HAIs (Jegers, Edbrooke, Hibbert, Chalfin, \& Burchardi, 2002). HAIs are a significant cause of increased length of stay (LOS) and increased costs for hospitalized patients. These costs are directly related to the type of HAI, resources needed to treat the infections, and the overall involvement of direct and indirect resource use needed during the course of these occurrences. HAIs represent $\$ 28$ to $\$ 33$ billion dollars in preventable healthcare expenditures yearly (Health and Human Services [HHS], 2009). In fact, hospital care for HAIs cost Medicare an estimated \$324 million just for the month of October of 2008 alone and extended LOS by an average of 19 days (Levinson, 2010; Lucado, Paez, Andrews, \& Steiner, 2010).

Development of HAIs is multifactorial and may include invasive diagnostic and therapeutic procedures, placement of invasive devices such as intravenous catheters, artificial airways such as endotracheal tubes used for mechanical ventilation, and urinary devices such as Foley catheters. These invasive devices provide a pathway for bacteria and other organisms to enter the bloodstream, the lungs, or the urinary tract increasing the risk of infection and other medical complications. The 3 most common and costly HAIs are Ventilator-Associated Pneumonia (VAP), Central Line-Associated Blood-Stream Infection (CLABSI), and Catheter-Associated Urinary Tract Infection (CAUTI). The high rate of HAIs is of great concern not for its impact on the health of ICU patients, but financially as well. Catheter-associated urinary tract infections account for $34 \%$ of all HAIs while CLABSI accounts for $14 \%$ (Klevens et al., 2007) and VAP for $15 \%$ 
respectively (Kieninger \& Lipsett, 2009). This study focused on the two most frequent HAIs.

Ventilator Associated Pneumonia (VAP). Ventilator Associated Pneumonia is developed by persons using a mechanical ventilator to assist or control respiration continuously through either a tracheostomy or an endotracheal tube. VAP develops within 48 hours of intubation (early onset) or during the weaning period (late onset) (Horan, Andrus, \& Dudeck, 2008). VAP, accounting for $15 \%$ of all HAIs, is frequently lethal, contributes greatly to increased health care costs, and accounts for higher indexes of morbidity and mortality (Kieninger \& Lipsett, 2009). Eighty six percent of healthcareassociated pneumonias are associated with mechanical ventilation (Koenig \& Truwit, 2006). Mortality rates range from $10 \%-70 \%$ increasing the likelihood of death 3 -to-4 fold, resulting in extended hospitalizations, excess use of antimicrobials, and increased direct medical costs up to $\$ 30,000$ per case (Coffin et al., 2008; Sole, Poalillo, Byers, \& Ludy, 2002). VAP is a leading cause of death in critically ill patients (Eachempati, Hydo, Shou, \& Barie, 2009).

Extremes of age (Coffin et al., 2008), supine position (Drakulovic et al., 1999; Grap, Munro, Bryant, \& Ashtiani, 2003; van Nieuwenhoven et al., 2006), etiology of oral and endotracheal aspirates (Bouza, Burillo, \& Muñoz, 2008; Munro, Grap, Jones, McClish, \& Sessler, 2009; Sole et al., 2002), type of endotracheal (ET) tube (Kollef et al., 2008), mechanical ventilation time (Rello et al., 2002), use of proton-pump inhibitors (Dodek et al., 2004), interruption of sedatives (Gerard et al., 2008), and antibiotic therapy (Chastre et al., 2003; Eachempati et al., 2009) have an effect in the prevention of VAP. Addressing these preventive measures in a compiled and simplified manner provides the 
baseline for care bundles designed to prevent and curtail VAP occurrences. Nursing has contributed significantly to the body of knowledge regarding prevention and implementation of interventions that prevent VAP. Research by Grap et al., $(2003 ; 2005)$ demonstrated that nursing interventions addressing back rest elevation affected the incidence of VAP.

Catheter-Associated Urinary Tract Infections (CAUTI). Urinary tract infections account for close to $40 \%$ of all healthcare-associated infections (Lo et al., 2008). Eighty percent of all urinary tract infections, the most common healthcare associated infection, are catheter-associated urinary tract infection (CAUTI) (Lo et al., 2008). Urinary catheters represent a daily risk of infection of 3\% to $5 \%$ when an indwelling catheter remains in situ (Lo et al., 2008) and are associated with increased death rates, prolonged hospital stays, and increased costs adding as much as $\$ 1,000$ to the direct cost of acute-care hospitalizations (Maki \& Tambyah, 2001). Urinary catheters are frequently used among critically ill patients in ICUs for accurate measurement of urinary output or for patients undergoing genitourinary surgeries requiring an invasive drainage. Reducing the length of time of indwelling catheterization, one of the most important modifiable risk factors for CAUTI, will reduce the mortality, length of stay, and cost associated with this condition (Lo et al., 2008; Maki \& Tambyah, 2001).

Research on the etiology of CAUTI has identified the advantage of closed drainage systems (Leone, Garnier, Dubuc, Bimar, \& Martin, 2001), early discontinuation of urinary catheter (Cravens \& Zweig, 2000), type of catheter (Brosnahan, Jull, \& Tracy, 2004), minimizing the use and appropriateness of urinary catheters (Elpern et al., 2009), and the efficacy of nurse-generated daily reminders to discontinue unnecessary urinary 
catheters in adult ICU patients (Huang et al., 2004) among the interventions that contribute to the prevention of CAUTI. Many institutions have adopted the recommendations of several agencies and organizations dedicated to promote prevention and elimination of HAIs. These recommendations have been operational and instituted as care bundles for HAIs including CAUTI. Contrary to the bundle for VAP, for CAUTI there is little consensus in the adoption of specific measures, and more criteria for its prevention is needed. Nurse-led interventions are critically important, not only in implementing the evidence based practice bundles, but generating data that supports these interventions. Fakih et al., (2008) reported the effect of nurse-led multidisciplinary rounds on reducing the unnecessary use of urinary catheterization in hospitalized patients. Despite the available body of knowledge, the challenge remains to continue improving best practices, developing tools to measure compliance with these practices, developing a gold-standard care bundle, and closing the knowledge gap on CAUTI.

\section{Pressure Ulcers}

ICUs also have a high incidence of pressure ulcer (up to $56 \%$ ) due to patient immobilization, use of sedation, and mechanical ventilation (Defloor, De Bacquer, \& Grypdonck, 2005; Keller, Wille, van Ramshorst, van der Werken, 2002; Schoonhoven, Defloor, \& Grypdonck, 2002). It has been estimated that from 1993 to 2006, total healthcare costs due to pressure ulcers were $\$ 11$ billion with the highest prevalence rates in ICUs (Keller et al., 2002; Russo, Steiner, \& Spector, 2008; Shahin, Dassen, \& Halfens, 2008; VanGilder, Amlung, Harrison, \& Meyer, 2009).

Pressure ulcers are skin localized areas where tissue necrosis has developed as a result of compression against a bony prominence or an external surface over a prolonged 
period of time (Jahnigen, Baxter, \& Bodenbender, 1989). Pressure ulcers are staged to facilitate identification and treatment of the resulting insult. There are currently four stages of pressure ulcers (Stage 1 through Stage 4) used to report the incidence and prevalence of these occurrences (Eachempati, Hydo, \& Barie, 2001). Pressure ulcers usually associated with undesirable patient outcomes, increased infection, additional surgical interventions, increased cost, and extended length of stay (Eachempati et al., 2001). Consequently, pressure ulcers are considered to be one of the most important indicators of the quality and effectiveness of patient care (Robinson, 2005). Reviewing the pathophysiology of pressure ulcers, the primary contributors are the amount and duration of pressure exerted on soft tissue (Reilly, Karakousis, Schrag, \& Stawicki, 2007). Poor nutrition (Eachempati et al., 2001), shearing force and friction (Bennett, Kavner, Lee, Trainor, \& Lewis, 1981), acute illness, and circulatory conditions or factors affecting tissue perfusion may also contribute to the development of pressure ulcers (Reilly et al., 2007).

Pressure ulcers have a negative impact on mortality, morbidity, and cost in ICU patients. The national pressure ulcer advisory panel suggested that the incidence and prevalence of pressure ulcer in critical care units was 22\% (National Healing Corporation, 2005). This incidence and prevalence has been reported to be higher in critically ill (de Laat, Schoonhoven, Pickkers, Verbeek \& van Achterberg, 2006). In an acute care facility, it was estimated that the cost of pressure ulcer treatment was $\$ 1300$ per patient or $\$ 80$ per day (Frantz, Gardner, Harvey, \& Specht, 1991). The majority of research studies have examined the incidence, prevalence, and risk factors on pressure ulcers in various healthcare settings (Bours et al., 2001; Pender \& Frazier, 2005; 
Pokorny, Koldjeski, \& Swanson, 2003). Despite the significance of pressure ulcer incidence on patient outcomes, minimal studies have examined it in ICUs. The limited literature available on pressure ulcers epidemiology in the ICU is largely from studies done in Europe, South Africa, South America, and Canada (Reilly et al., 2007).

Additional Factors Affecting ICU Length of Stay, Patient Outcomes, and Healthcare

\section{Costs}

Patient-Initiated Removal of Devices. Patient-initiated device removal is not uncommon in ICU settings. This unplanned and premature removal of these devices (i.e., endotracheal tubes, indwelling urinary catheters, nasogastric tubes, and small bowel feeding tubes) may lead to injury, harm, increased cost, prolonged LOS, and even death (Carrion et al., 2000; Mion et al., 2001; Mion, Minnick, Leipzig, Catrambone, \& Johnson, 2007). As a result of these unplanned removals of therapeutic devices, studies and procedures such as radiographs, suturing, reinsertion of dislodged devices, laboratory work, and other invasive interventions may be required (Mion et al., 2007). Studies on the patient-initiated removal of devices in the ICU setting estimated for self-extubation range from less than 50 to 125 episodes per 1,000 patient- days (Carrion et al., 2000; Mion et al., 2001, 2007). The frequency of accidental extubation was reported to range from 3 to 16\% 1990s (Carrion et al., 2000). However, these studies were conducted in one or two hospitals for short observational times (Carrion et al., 2000; Fraser, Riker, Prato, \& Williams, 2001; Mion et al., 2001). Similarly, accidental removal of nasogastric tubes was estimated to be 26 to $41 \%$ (Carrion et al., 2000). The negative impact of these device removals on patient outcomes may range from a delay in therapy, to respiratory 
distress, and urinary retention to more severe complications such as hemorrhage, pneumothorax, vocal cord damage, and death (Mion et al., 2007).

Morbidity rates from patient-initiated removal of therapeutic devices have been reported as high as 23\% (Frezza, Carleton, \& Valenziano, 2000; Mion et al., 2007; Moons, Sels, De Becker, De Guest, \& Ferdinande, 2000). The cost of these patientinitiated therapeutic device removals has been estimated as high as more than $\$ 250,000$ a year or $\$ 181$ per event (Fraser et al., 2001). Carrion and colleagues (2000) have suggested the importance of surveillance of medical personnel and education in reducing patient-initiated accidental removal of devices.

Sedation Management. Management of sedation in the ICU requires advanced skills in assessing patient needs to address agitation and titration of therapy targeted to achieve specific sedation levels (Kress \& Hall, 2006; Sessler, Grap, \& Brophy, 2001). Critically ill patients require frequent monitoring to assess levels of sedation thus patient focused titration of sedation and specific end-points are needed (Sessler, Grap, \& Ramsay, 2008). Generally, the aim of sedation protocols in ICU patients is to decrease the use and duration of sedatives during ICU stays. Nursing-directed protocols emphasizing the use of intermittent therapy (e.g., the use of sedatives as needed) have shown shorter duration of mechanical ventilation (56 hours vs. 117 hours), shorter ICU LOS (5.7 days vs. 7.5 days), and shorter overall hospital LOS (14.0 days vs. 19.9 days) (Brook et al., 1999). De-escalation therapy (e.g., stopping continuous infusion daily for short periods of time until the patient is able to follow several simple commands or become agitated) from continuous infusions to intermittent therapy demonstrated shorter duration of mechanical ventilation (Brook et al., 1999). These findings led Kress, 
Pohlman, O'Connor, and Hall (2000) to test daily interruptions of sedative infusions in critically ill patients undergoing mechanical ventilation. The authors demonstrated that the use of "sedation vacation" significantly reduced the duration of ventilator use and ICU LOS (Kress et al., 2000). Schweickert, Gehlbach, Pohlman, Hall, and Kress (2004) showed that daily interruption of sedative infusions in ICU patients reduced ICU LOS and decreased the incidence of complications associated with prolonged intubation and mechanical ventilation.

Although appropriate management of sedation was found to reduce side effects of medication, decrease duration of mechanical ventilation, shorten ICU and hospital LOS, decrease ICU-related complications, and reduce overall costs of hospital stay (Brook et al., 1999; Carson et al., 2006; Chanques et al., 2006; Kress et al., 2000; MacLaren \& Sullivan, 2006; Mascia, Koch, \& Medicis, 2000; Richman, Baram, Varela, \& Glass, 2006) few studies have examined the role of APNs in managing sedation in the ICU. APN Intensivists in certain ICUs have enforced patient-focused assessment of predisposing and precipitating agitation factors, medication selection, patient monitoring, and strategies targeting therapy to minimize excessive or prolonged sedation administration. However, the contribution of these APN Intensivists has not been documented.

Post-Surgical Glycemic Control. Acute hyperglycemia has been defined as the random glucose concentration of more than $140 \mathrm{mg} / \mathrm{dl}$ (American Diabetes Association [ADA], 2010). Stress hyperglycemia is the increase of blood glucose during an episode of acute illness (Dungan, Braithwaite, \& Preiser, 2009) and has been associated with a plethora of factors including stress hormones, use of glucocorticoids, and sepsis all 
inhibiting insulin release and action during acute illness or surgical trauma (Kavanagh \& McCowen, 2010). Hyperglycemia has been associated with increased mortality in critically ill patients (Krinsley, 2008). Controlling a low degree of glycemic variability may significantly decrease mortality and improve patient outcomes in ICUs implementing glycemic variability metric (Krinsley, 2008). The optimal target goals of glucose levels in ICU patients remain uncertain (Kavanagh \& McCowen, 2010). Recently, the American Association of Clinical Endocrinologists and American Diabetes Association recommended target glucose levels for ICU patients between 140 to 180 mg/dl (ADA, 2010). Other organizations (i.e., Surviving Sepsis Campaign [SSC], Institute for Healthcare Improvement [IHI], 2011b) suggested target glucose levels of 150 mg/dl to less than $180 \mathrm{mg} / \mathrm{dl}$ respectively. Since Van den Berghe and colleagues' (2001) study on surgical ICU patients showed decreased ICU and ventilator days, blood stream infections, renal failure, and costs, much controversy has been aroused because of the possible harm of intensive insulin therapy resulting in hypoglycemic events (Bochicchio \& Scalea, 2008). Nevertheless, glycemic control has been demonstrated to improve outcomes in critically ill patients. Finfer et al. (2009) challenged the results reported by Van den Berghe and colleagues (2001) and suggested that intensive insulin therapy resulting in hypoglycemia did not show benefits in mortality rates but conceded some benefits to patients in surgical ICUs.

A study to examine the effect of an intensive glycemia management protocol on the cost of care of critically ill adult patients suggested a net annual decrease of $\$ 1,339,500$ or $\$ 1,580$ per patient. These findings showed a decrease in ICU LOS, ventilator days, costs (i.e., laboratory, pharmacy, and radiology), and post-ICU hospital 
LOS (Krinsley \& Jones, 2006). Despite the controversies surrounding management of glycemic control in the ICU population, interventions aiming to control glycemia in surgical ICU patients have demonstrated reduced hospital-acquired infections and postoperative complications (Grey \& Perdizet, 2004) as well as need for dialysis.

Ventilator Weaning and Ventilator Days. Weaning refers to the process of liberating patients from mechanical ventilation and ultimately resulting in the removal of the endotracheal tube in intubated patients. Research on weaning has documented its contribution to mortality, morbidity, and cost (Cook et al., 2000; Ely et al., 2000). The appropriate timing of weaning and extubation is crucial to prevent VAP, failed extubation, or increased mortality (Epstein, Ciubotaru, \& Wong, 1997). The greater the number of days ( 24 hours) on the ventilator, the greater the incidence of patient morbidity (Beale, Bryg, \& Bihari, 1999; Fink, Krause, Barrett, Schaaff, \& Alex, 1998; Valles et al., 1995). Extubation failures have been documented in 2 to $25 \%$ of mechanically ventilated patients (Epstein, Nevins, \& Chung, 2000). ICU patients have the highest rates of reintubation (Rady \& Ryan, 1999). In a longitudinal study of outcomes of 210 critically ill patients undergoing prolonged mechanical ventilation at an acute care respiratory unit, researchers found that a substantial portion of these patients were unable to be weaned from ventilator use, resulting in increased mortality (Bigatello, Stelfox, Berra, Schmidt, \& Gettings, 2007). Nurse and physician staffing in the ICU were found to affect the rates of reintubation in ICU patients undergoing esophageal resection and abdominal aortic surgery (Dang, Johantgen, Pronovost, Jenckes, \& Bass, 2002; Amaravadi, Dimick, Pronovost, \& Lipsett, 2000; Pronovost et al., 1999). 


\section{Healthcare Charges}

Economic evaluation of resource use in hospitals is often reported as either microcosts or as charges (Scott, 2009). Micro-costs are the expenses incurred by hospitals in goods and services. In other words, a cost is derived for each step of an intervention (e.g., staff time, supplies, medications, and out-of-pocket expenses) (Smith, Barnett, Phibbs, \& Wagner, 2010). Micro-costs provide a more precise estimate of the economic value of the resources used in hospital care (Scott, 2009). However, the use of hospital charges to estimate cost may be imprecise due to cost-shifting or overestimation (Finkler \& Getzen, 2008). If micro-costing is not feasible, cost estimates based on adjusted costs or using cost-to-charge ratios like Centers for Medicare and Medicaid Services (CMS) does may suffice.

The economic impact of patient complications is critical since the extra costs associated with these conditions are usually not matched by increased revenues when Medicare is the payer (Pyenson, Murphy-Barron, Mirkin, \& Iwasaki, 2007). This may also be true for some private insurers that tend to follow the Medicare reimbursement practices. This is particularly true when considering that CMS will no longer reimburse additional expenses for specific conditions named never events (e.g., HAIs, pressure ulcers).

\section{Length of Stay (LOS)}

Length of stay, the number of 24-hour calendar days for inpatients, is measured from admission to discharge from the hospital or a unit within the hospital such as the ICU. This measure can be used as an indicator for quality of care. Length of stay in ICUs can be linked to costs, mortality, and morbidity (Gruenberg et al., 2006). 
Observational studies in ICUs report LOS not only as a measure of quality, but also as a predictor of patient outcomes. Length of stay findings compounded with other quality measures usually serve as the groundwork for initiatives in ICUs procuring enhanced patient outcomes, decreased morbidity and mortality, and decreased cost (Fuchs, Berenholtz, \& Dorman, 2005; Pronovost et al., 1999).

\section{Types and Levels of ICU Staffing}

Hospitals across the U.S. have adopted either Open, Closed, or Semi closed models of ICUs. Open ICUs are ICUs where any attending physician with hospital admitting privileges can direct patient care in the unit (Carlson et al., 1996). Closed ICUs are units where the care is coordinated and provided by trained physician intensivists (physicians who specialize in the care of critically ill patients) either directly or by directing care as the head of the multidisciplinary team (Brill et al., 2001). Transitional or Semi closed ICUs, are units where the physician intensivists are present in the unit and available to provide care but care is shared and co-managed with other physicians such as surgeons or internists (Brill et al., 2001). Anesthesiologists, surgeons, and internists are now collaborating in the management of patients admitted to ICUs. Members of other health care disciplines are also integrating their services in multidisciplinary teams responsible for the delivery of care in ICUs.

ICUs that use a physician intensivist model of care have improved patient outcomes (e.g. decreased HAIs) (Zell \& Goldmann, 2007), less resource use, and lower mortality rates (up to a $40 \%$ reduction) (LeapFrog Group, 2008). Young \& Birkmeyer (2000), found reductions in mortality rates from $15 \%$ to $60 \%$ in ICUs using intensivists. When evaluating the effect of an intensivist-model of critical care delivery on the risk of 
death following injury, researchers found that care in an intensivist-model or closed ICU was associated with a large reduction in in-hospital mortality following trauma, particularly in the elderly (Nathens et al., 2006). Implementing this intensivist model in critical care settings will help save almost 54,000 Americans in the U.S. each year (Young \& Birkmeyer, 2000).

By the year 2020 an estimated $22 \%$ shortage of intensivists is expected as a result of the aging population and a growing demand for intensivists. This will have increased to an approximate $35 \%$ shortage of intensivists by 2030 (USDHHS HRSA, 2006). In Europe and other countries the responsibility of managing ICU patients remains largely in the hands of physician intensivists, whereas in the U. S. more of this care is conducted by multidisciplinary teams (Hanson et al., 2001) and not necessarily headed by physician intensivists. However, who assigns responsibilities for care of ICU patients and who should lead such efforts remain points of controversy in the U.S. and abroad. Pronovost et al., (2002) examined staffing patterns versus clinical outcomes and found that staffing ICUs with intensivists was associated with reduced length of stay, and reduced hospital and ICU mortality. Research also indicates that intensivists provide the most optimal level of care in critical care. They are more likely to practice in large hospitals. Over 5 billion dollars in healthcare costs could be saved annually by implementing changes in physician staffing just in non-rural U.S. hospitals (Pronovost et al., 2002). The compounded effect of this projected shortage of physician intensivists and physicians practicing in critical care in smaller hospitals may be larger. This shortage will negatively impact small hospitals and rural communities putting more at risk certain vulnerable populations (USDHHS, HRSA, 2006). 
The shortage of intensivists will create difficulty in providing requirements for 24-hour on-site coverage in ICUs by these professionals, resulting in potential burnout that may contribute to their early retirement from the work force (USDHHS, HRSA, 2006). In addition there is the impact of the mandated 80 -hour work-restrictions for general surgical residents mandated by the Accreditation Council of Graduate Medical Education (ACGME) and the Residency Review Committee (RRC) in the U.S. (Accreditation Council for Graduate Medical Education [ACGME], 2004). These work restrictions add an extra burden on who can provides 24-hour ICU in-house coverage (Gordon, Axelrad, Alexander, Dellinger, \& Ross, 2006). A study by Gordon et al., (2006) revealed that $37 \%$ of medical training programs surveyed have residents other than general surgery residents covering the ICUs. Around 30\% of these surveyed programs used physician assistants or APNs to cover the ICUs during daytime and 11\% of the programs used physician assistants or APNs to cover the ICUs during nighttime (Gordon et al., 2006).

Mitchell, Ashley, Zinner, and Moore's (2007) study showed that a reduction of 20 hours in resident duty hours per week will require the hiring of 10 physician assistants at a cost of $\$ 1,134,000$ to cover this void. The demand for ICU services is exceeding the supply as a result of the growth of the elderly population and the attrition of physician intensivists (Ewart et al., 2004). To meet this demand, the government, insurers, administrators, and providers must look for viable alternatives to provide quality care cost effectively in management of critical care patients. Reports on Medicare have predicted increased costs and demand for services as the U.S. population ages; however how to deliver these services is still unclear (Angus et al., 2000). 
In contrast to the shortage of intensivist physicians and critical care trained physicians, APNs, sometimes referred to as mid-level practitioners or non-physician providers, are increasing in acute care both in the United States and globally (Sheer \& Wong, 2008). In the U.S., APNs have increased in numbers and expanded their roles over the past decades, becoming an important link in healthcare systems and organizations. The APN designation includes clinical nurse specialists (CNSs), nurse practitioners (NPs), nurse midwives (CNMs), and nurse anesthetists (CRNAs). They have completed graduate-level education, obtained certification from a national certification program, practiced focusing on direct patient care, and have acquired an advanced level of competencies, skills and autonomy (APRN Joint Dialogue Group, 2008). APNs are licensed independent practitioners prepared to provide services for their specialty population across the health wellness-illness continuum. The largest group of APNs is nurse practitioners. There are over 97,000 NPs registered in the U.S. as of 2004 (Goolsby, 2005). Some APNs function as Acute Care Nurse Practitioners (ACNPs) in hospitals and in ICUs. In spite of this increased visibility and participation in the healthcare arena, healthcare providers and potential employers are still unfamiliar with the scope of practice of APNs (McLaughlin, 2007).

Since the early 1980s, the role of APNs in providing high quality care and low cost has been documented (Brooten et al., 1986). Several other studies have documented the role of APNs in different practice areas providing high quality care and low cost thus benefitting the patient, provider, and healthcare system (Brooten et al., 1986, 1994, 2002; Hoffman, Tasota, Zullo, Scharfenberg, \& Donahoe, 2005; Kleinpell, 2005; Rudy et al., 1998; Sidani et al., 2006; Spisso, O’Callaghan, McKennan, \& Holcroft, 1990). Care 
provided by APNs in acute care has been documented in numerous studies examining quality of care, (Kleinpell \& Gawlinski, 2005), morbidity, and mortality (Gracias et al., 2008; Hoffman et al., 2005; Munro \& Taylor-Panek, 2007; Russell, VorderBruegge, \& Burns, 2002; Sole, Hunkar-Huie, Schiller, \& Cheatham, 2001; Burns et al., 2003; Meyer $\&$ Miers, 2005). Results of these studies have indicated that the quality of care provided by APNs is equal to that of physicians as well as being cost efficient (Horrocks, Anderson, \& Salisbury, 2002; Kinnersley et al., 2000). However, studies in the ICU documenting the role of APNs in reducing the costs of care on specific patient outcomes are very limited.

The evolution of the role of APNs in acute care has responded to an increased need for tertiary services, the shortage of medical staffing to respond to these needs, and the advancement of the role of APNs in clinical practice. In ICUs, the role of APNs has been evolving to replace physicians and support patient care rather than providing traditional nursing care (Knaus, Felten, Burton, Forbes, \& Davis, 1997; Mundinger, 1999). APNs function as members of multidisciplinary teams in closed (intensivist lead) or semi-closed (intensivist shared lead) ICUs. Collaborative practice rather than individual or autonomous practice is the norm in both instances. However, APNs have continued to enjoy autonomy in decision making and treatment modalities as permitted by the scope of their respective collaborative agreements. The APN Intensivist is a healthcare provider with a high level of independence and specialization in the management of critically ill patients in critical care settings. Very little has been published on this relatively new sub-specialty role of APNs. The lack of literature to explain the role of APNs as intensivists and of research to evaluate the effectiveness of 
this APN role made the need clear for research to examine the role economically and for its effect on quality of care and patient outcomes.

In summary, research has identified and extensively documented the contributions of APNs on patient outcomes and healthcare systems. However, the lack of supporting evidence on the effects of APNs as Intensivists remained. Despite the magnitude of ICU complications, there is a dearth of literature documenting the effect of APN intensivists in curtailing the incidence of HAIs, pressure ulcers, patient-initiated removal of therapeutic devices, managing and controlling glycemic levels in ICU patients, and on ventilator weaning. In light of current legislation, input substitution (i.e., allowing the substitution of NPs for MDs) as an alternative in providing care (Laurant et al., 2006) and policies to find cost effective alternative models of healthcare delivery, the need to document the effects of this model of ICU staffing was evident. Other factors such as the growing aging population, physician intensivist attrition, and rise of healthcare costs supported the need for this research.

\section{Study Purpose}

The purpose of this study was to examine the dose effect of APN Intensivists in a surgical intensive care unit (SICU) on patient outcomes, healthcare charges, APN Intensivists services, and APNs special initiatives when APN staffing differed.

1. Was there a difference in patient outcomes (HAIs [i.e., VAP, CAUTI], pressure ulcers, patient-initiated accidental removal of devices [i.e., selfextubation, removal of venous access, removal of urinary catheters, and removal of feeding tubes], sedation management, post-surgical glycemic 
control, and ventilator weaning and ventilator days) and SICU length of stay [LOS] when the ICU was staffed by:

a. Two APN Intensivists per shift 24/7 (around the clock) compared to zero to one APN Intensivist per day (2009 [T1] vs. 2011 [T4]).

b. One APN Intensivist per shift compared to zero to one APN Intensivist per day (January to June 2010 [T2] to 2011 [T4]).

c. One APN Intensivist per shift but not for seven days compared to zero to one APN Intensivist per day (July to December 2010 [T3] to 2011 [T4]).

2. Was there a difference in health care charges for SICU length of stay and APN Intensivists services when the ICU was staffed by:

a. Two APN Intensivists per shift 24/7 (around the clock) compared to zero to one APN Intensivist per day (2009 [T1] vs. 2011 [T4]).

b. One APN Intensivist per shift compared to zero to one APN Intensivist per day (January to June 2010 [T2] to 2011 [T4]).

c. One APN Intensivist per shift but not for seven days compared to zero to one APN Intensivist per day (July to December 2010 [T3] to 2011 [T4]).

3. Was there a difference in the frequency of APN Intensivist special initiatives conducted over the four time periods (i.e., T1, T2, T3, and T4) over the 3-year study period between 2009 and 2011 ? 


\section{Conceptual Framework}

The theoretical framework that guided this study is based on the work of Donabedian (2005). This framework has used the paradigm "structure-process-outcome" for the assessment of quality of care, and has influenced health care for over 40 years. The model has been particularly instrumental in grounding outcomes as indicators for the quality of "antecedent care" (Donabedian, 1978). Donabedian emphasized the importance of the healthcare structure as a driving force for care processes that resulted in better healthcare outcomes (Donabedian, 1988). Process measures of quality are defined as the activities performed by healthcare professionals in treating patients (Donabedian, 1988). Outcome measures are the results of these care processes (Donabedian, 1988).

Donabedian methods for assessing and monitoring the quality of care for research represented the most appropriate conceptual framework for assessing the structures, processes and patient outcomes of APN intensivist care. Structures of healthcare delivery are referred to the conditions under which care was being provided. In this study, the structure was Jackson Memorial Hospital Surgical Intensive Care Unit where critically ill post-surgical adult patients are admitted for care. Process referred to the way healthcare providers, in this study APN Intensivist delivered care. Outcomes were defined as those changes in health status attributed to the care provided. In this study the outcomes included patient outcomes (HAIs [i.e., VAP, CAUTI], pressure ulcers, SICU length of stay [LOS], patient-initiated accidental removal of devices [i.e., self-extubation, removal of venous access, removal of urinary catheters, and removal of feeding tubes], sedation management, post-surgical glycemic control, ventilator days and ventilator weaning, and bladder catheter days), healthcare charges, APN Intensivists services, and APNs special 
initiatives. The framework in Figure 1 illustrates the relationship between process and outcome.

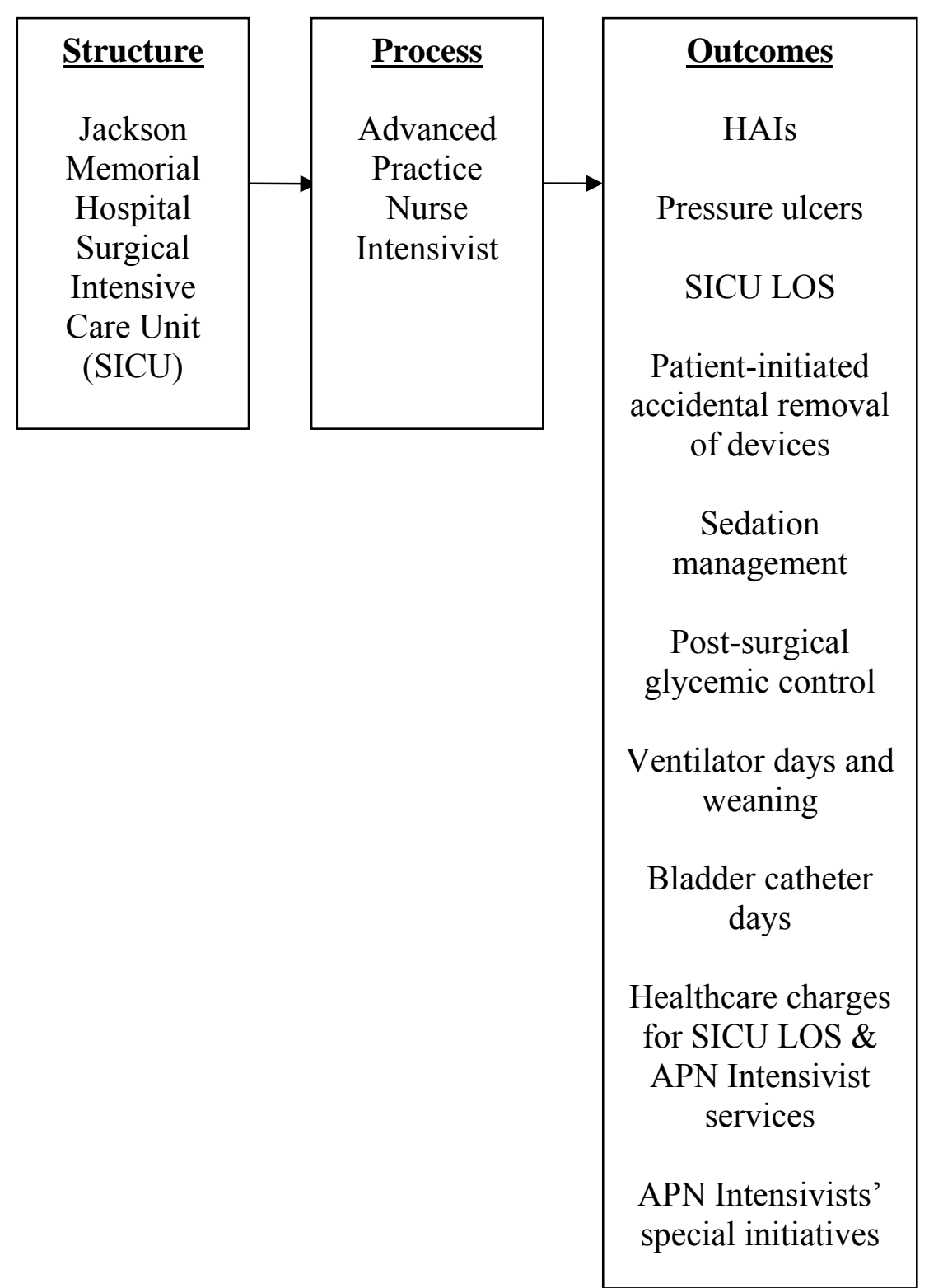

Figure 1. Donabedian's Framework 


\section{CHAPTER II}

\section{Literature Review}

\section{Introduction}

In order to examine the effects of APN Intensivists in Intensive Care Units on patient outcomes, healthcare costs and implementation of quality initiatives, an understanding of their role and of the circumstances in which their practice is exercised is

necessary. This chapter provides a review of the literature in the following general areas:

1. Literature on the structure and staffing of ICUs.

2. Literature related to Advance Practice Nurses, APNs in acute care and APN Intensivists.

3. Literature related to patient outcomes, including HAIs, pressure ulcers, ICU LOS, patient-initiated accidental removal of devices, sedation management, post-surgical glycemic control, ventilator days, ventilator weaning and bladder-catheter days.

4. Literature related to healthcare costs and charges for ICU stays.

5. Literature related to special initiatives.

\section{Structures of Intensive Care Units (ICUs)}

Development of ICU Care. ICUs account for over 10 percent of all hospital beds in U.S. representing over 4.4 million individual patient admissions yearly (USDHHS, HRSA, 2006). Today’s ICU is a challenging stronghold of technology, advanced therapy modalities, innovative diagnostic and therapeutic interventions, and leads in sophisticated care (Grenvik \& Pinsky, 2009). However, the evolution of the intensive care unit as a specialized clinical unit and the evolution of critical care medicine 
as a specialty may date back as far as the nineteenth century. A nurse, Florence Nightingale, is considered to be the first to have used an ICU model of care for injured soldiers in the battlefield (Grenvik \& Pinsky, 2009).

No reports of similar model use were recorded until 1929 when a special postoperative unit for neurosurgical patients was developed at John Hopkins Hospital in Baltimore, U.S. (Hanson et al., 2001). Two decades later, during the worldwide outbreak of poliomyelitis in the 1950s, intensive care unit prototypes started flourishing. This was the beginning of organized high-acuity level intensive care units. Compelling evidence points to Kommunehospitalet in Copenhagen, Denmark, as the first established intensive care unit worldwide (Berthelsen \& Cronqvist, 2003). The need for a high level of respiratory care for patients with bulbar poliomyelitis and the advent of mechanical ventilation and improved respiratory support helped redefine the intensive care unit model. Intensive care medicine may owe its origins to the need for supporting failing ventilation (Grenvik \& Pinsky, 2009).

In later decades, with the advancement in the fields of cardiac and neurosurgery, trauma and burns management, and the innovation of the biomedical technology world, critical care units became highly sophisticated specialized units. Most ICUs were initially medical (MICU) or surgical (SICU) ICUs (Grenvik \& Pinsky, 2009). Thereafter, the emergence and proliferation of pediatric intensive care unit (PICU), neonatal intensive care unit (NICU), trauma intensive care unit (TICU), medical intensive care unit (MICU), surgical intensive care unit (SICU), and coronary care unit (CCU) occurred in the U.S. and globally. 
In the United States, trends to isolate ICUs by specialties such as surgery, trauma, cardiothoracic, and others occurred in large tertiary care hospitals (Grenvik \& Pinsky, 2009). This segregation created the need to integrate physicians from different specialties in the management of these units. Anesthesiologists, surgeons, and internists are now collaborating in the management of patients admitted to these highly specialized units. Members of other healthcare disciplines are also integrating their services in creating a multidisciplinary team responsible for delivery of care in these units.

A multidisciplinary approach needed to address clinical challenges helped shape the structure and organization of these units. The team model has been advocated as the optimal method to deliver care in the ICUs (Durbin, 2006). Resulting collaboration has been documented extensively and places the intensivist at the helm of this enterprise (Durbin, 2006). Nursing as a discipline has also been instrumental in the development and specialization of ICUs and critical care medicine (Binnekade, Vroom, de Mol, \& de Haan, 2003). ICU nurses are an essential link in the multidisciplinary team model responsible for planning and integrating the care of patients in these units.

Staff nurses have become specialized and trained in critical care (Durbin, 2006). These critical care nurses have become more specialized and specifically trained in these specialized areas of practice. Staffing of these units with highly skilled nurses ensures optimal care and safety. Published literature documents the role of nurses' education and qualifications, and staffing ratios in improved patient outcomes (Aiken, Clarke, Cheung, Sloane, \& Silber, 2003; Aiken, Clarke, Sloane, Sochalski, \& Silber, 2002; Blegen, Goode, \& Reed, 1998; Cho, Ketefian, Barkauskas, \& Smith, 2003; Clarke \& Aiken, 2003; Giraud et al., 1993; Lichtig, Knauf, \& Milholland, 1999; McGillis Hall, Doran, \& 
Pink, 2004; Needleman, Buerhaus, Mattke, Stewart, \& Zelevinsky, 2002a; Needleman, Buerhaus, Mattke, Stewart, \& Zelevinsky, 2002b; Person et al., 2004; Pronovost et al., 2001; Pronovost et al.,1999). The effect of nurse staffing and antimicrobial-impregnated central venous catheters on the risk of acquiring bloodstream infections in intensive care units has also been documented (Alonso-Echanove et al., 2003).

The ICU multidisciplinary team includes technical support provided by professionals including respiratory and telemetry technicians. Over the time, other disciplines have been incorporated including; occupational and physical therapists, social workers, pharmacists, nutritionists, and ethicists. Their inclusion has provided a more thorough approach to meeting critical care patients' and families' needs.

In summary, specialized ICUs are now the model used at large urban and university medical centers, and hospitals. However, rural and community hospitals continue providing critical care and specialized intensive care to patients in units designated as ICUs that do not necessarily follow the models used in large urban tertiary care settings. Globally, the responsibility of managing ICU patients in Europe remains largely in the hands of physician intensivists, whereas in the U. S. such management is usually multidisciplinary (Hanson et al., 2001) and are not necessarily headed by physician intensivists.

Physician Providers. The role of physician intensivists, physicians who specialize in the care of critically ill patients usually in an ICU, has been explored, documented, and argued in favor of this model for improved patient outcomes. Understanding their role in modeling ICU care is essential to evaluate alternatives or substitutes of their care. This section explores the development of intensive care units 
(ICU) and critical care related to physician intensivists' models of ICU care and the effects on patient outcomes and healthcare costs. Understanding the development and implementation of these models and their impact on the health, economy, and welfare of citizens may facilitate the dissemination of these models. Currently in intensive care units not managed by physician intensivists, approximately 360,000 deaths occur annually (USDHHS, HRSA, 2006). There is a projected $22 \%$ shortage of intensivists by the year 2020 and a $35 \%$ projected shortage by 2030 , as a result of the aging population and a growing demand for intensivists (USDHHS, HRSA, 2006). The impact on the underserved is particularly acute.

Several studies have advocated for the physician intensivist model (Hanson et al., 1999; Pronovost et al., 1999; Pronovost et al., 2002; Reynolds, Haupt, Thill-Bahazorian, \& Carlson, 1988) in ICUs. Durbin (2006), in advocating for care in ICUs through an intensivist-lead Team Model, pointed to an estimated 30\% - 50\% reduction in mortality when intensivists rounded daily on critically ill patients, and to the shortening of the length of stay, reduction in cost, and prevention of complications with the implementation of this model. However the role of physician intensivists remains a source of debate among practicing critical care medicine physicians and throughout the medical profession in general. What is an intensivist and who should provide ICU care are the core questions in this debate. In addition, the dearth of a universally accepted paradigm for critical care and the relatively short existence of the specialty (Hanson et al., 1999) may add to the complexity of this debate. 
In 1992 the Society of Critical Care Medicine (SCCM) issued guidelines describing the functions and requirements of an intensivist (Guidelines Committee, Society of Critical Care Medicine, 1992). A physician intensivist was defined as: "a physician trained and certified through a primary specialty that successfully completed an Accreditation Council for Graduate Medical Education-approved training program in critical care medicine and/or has a certificate of special qualification in critical care" (SCCM, 2006).

The physician intensivist is responsible to diagnose, manage, monitor, intervene, and individualize the care of critically ill patients and patients in the immediate perioperative period. The physician intensivist should be able to perform, manage, and coordinate the need of procedures such as maintenance of airway patency, endotracheal intubation, placement of intravascular catheters, pacing devices, cardiopulmonary resuscitation, thoracotomies, and other procedures. In addition the physician intensivist should be immediately and physically available to patients in the ICU, participate in 24hour coverage of the ICU, and promote and maintain the efficient use of resources, provide education, and management of the ICU. The physician intensivists are also responsible for unit-based data collection and performance improvement activities.

Historically, the debate between the specialties of anesthesiology, medicine, and surgery has revolved around who should lead the care of ICU patients, and exert management control of ICUs. This debate continues. Any of the specialists claims $a$ priori may underestimate the contributions that each made to critical care and specifically to the development of ICU care. Additionally, these disciplines share so many 
commonalities in scope of practice and qualifications which complicates the boundaries of each in claiming ownership.

The role of the anesthesiologist and that of Anesthesia as a specialty has been important in the development of critical care and ICUs in the U.S. Anesthesiologists were at the forefront of critical care in the U.S. during World War II when surgical casualties were grouped in shock wards (Hanson et al., 2001). Later, their role in postoperative recovery rooms (which may have been the precursors of surgical intensive care units, and in other respiratory care units has been prominent within critical care (Hanson et al., 2001). Approximately $6 \%$ of the total intensive care delivered in the U.S. is in the hands of anesthesiologists, of which $60 \%$ are certified in critical care (Angus et al., 2000).

Although the number of practicing anesthesiologist physicians in critical care is relatively low in comparison to other specialties (i.e.; pulmonologists [79\%]) (Angus et al., 2000), their contributions to the field of intensive care medicine through research and practice is well documented. Most of the evidence on the role of physician intensivists in improving patient outcomes has been generated by anesthesia intensivists (Hanson et al., 2001). However, the role of anesthesiologists, specifically in critical care, is unknown to the public and to other medical specialties.

The anesthesiology leadership must promote the role of the anesthesiologist as intensivists, to extend their practice into ICUs' management, and in the field of critical care. As a physician intensivist, anesthesiologists' knowledge in acute pathophysiology, advanced pharmacology, hemodynamics, and skill in airway management is recognized. 
The argument in favor of them assuming responsibility for ICU patients and critical care in general, is based on their knowledge and skill in these areas.

Surgeons and internal medicine physicians (particularly pulmonologists), argue over control of management of patients in the ICU and ICUs in general. For these physicians, completion of training in critical care, as for anesthesiologists, is required and board certification in their specialty is a pre-requisite. Provision of at least six weeks of full-time ICU care annually is expected to maintain certification. Whether the care rendered by intensivists in ICU differs from that of physician specialists or members of other healthcare disciplines has not yet been studied. What has been documented is that ICUs managed by intensivists have better patient outcomes (Pronovost et al., 1999).

Surgical intensivists (a surgeon in a critical care clinical role) are able to provide complex care to surgical intensive care patients and to address problems and conditions that require advanced technical modalities and sometimes emergent surgical intervention. Having these skills make them the ideal candidate to manage critically ill patients in surgical ICU settings. Surgical critical care medicine and Surgical Intensive Care Units (SICUs) have evolved over the years increasing the number of intensivists practicing at large teaching hospitals and urban medical centers. Perhaps, this ability to perform in both roles, as surgeons and as clinicians may grant them a slight advantage over the other medical disciplines, namely anesthesiology and internal medicine. However, research supporting these assumptions are limited (Pronovost et al. 1999).

The attrition of intensivist and critical care specialists expected over the next ten years poses an incredible risk to the welfare of our citizens. Currently there are only enough board-certified intensivists to staff approximately $25 \%$ of the ICU beds (Angus et 
al. 2000). The shortage of intensivists and other physicians practicing in critical care is putting vulnerable populations at risk (Angus et al. 2000; Burchardi \& Moerer, 2001).

Burchardi and Moerer (2001) concluded that the shortage of intensivists will increase the difficulty in meeting requirements of 24-hour on-site coverage for ICUs by these professionals. Burnout may also contribute to physician intensivists' attrition (USDHHS, HRSA, 2006). In addition, the impact of the mandated 80-hour workrestrictions for general surgical residents mandated by the Accreditation Council of Graduate Medical Education (ACGME) and the Residency Review Committee (RRC) in the U.S. in July 2003 also affects availability of physician intensivists for ICU coverage (ACGME, 2004).

These work restrictions added an extra burden to who provides to and facilitates 24-hour ICU in-house coverage (Gordon et al. 2006). Interestingly, the results of their study (Gordon et al. 2006) revealed that $37 \%$ of the programs surveyed have residents other than general surgery residents covering the ICUs, and most importantly, that $30 \%$ of the programs surveyed used physician assistants or APNs to cover the ICUs during daytime and $11 \%$ at nighttime (Gordon et al., 2006). One study revealed that a reduction in resident duty hours per week will require the hiring of 10 physician assistants at a cost of $\$ 1,134,000$ to cover this void (Mitchell et al. 2007).

This unprecedented shortage of critical care services by physicians is of utmost importance. The compelling evidence of exceeded demand versus supply as the result of the growth of the elderly population for instance (Ewart et al. 2004) should motivate the government to look for viable alternatives to provide quality care, and cost effective management of patients in critical care. Reports on Medicare have predicted increased 
costs and demand for services as the U.S. population ages, but a lack on how to deliver these services is still present (Angus et al. 2000).

In summary, we are not only facing the lack of a standardized criteria for who should practice critical care and where, but in addition we are still struggling to standardize the implementation of an intensivist-centered model across ICUs. To that effect, the three most common models of ICU care delivered in the U.S. to date are the Close, Open, and Semi closed units. To understand the extent of this problem and the impact on healthcare one needs to understand first the organizational characteristics s of these intensive care units.

Open versus Closed or Semi Closed Units. In general terms, hospitals across the U.S. have either Open, Closed, or Semi closed models of ICUs. Over 54,000 deaths yearly can be prevented if hospitals adopt ICUs managed by intensivists (Closed unit model) (Birkmeyer \& Dimick, 2004). Almost 1\% of the U.S. gross domestic product is consumed by ICU patients (Halpern \& Pastores, 2010).

Assuming that the physician intensivist leads and coordinates ICU management, ICU activities and culture are then defined by the extent to which these intensivists are involved in such practices. This premise allows for categorizing such units into Open, Closed, and Semi-closed or Transitional units. This distinction intrinsically facilitates and generates research opportunities comparing models and evaluating differences resulting from such comparisons.

Open ICUs are intensive care units where any attending with hospital admitting privileges can direct patient care in the unit (Carlson et al., 1996). Orders may be written by any licensed physician with privileges regardless of their specialty or training. 
Contrastingly different, Closed units are directed by an intensivist physician. But the differences between these two models are not limited to who is in charge of directing patient's care and who is responsible for the coordination of services. Intrinsically, the differences reach beyond roles and functions.

Closed ICUs are units where the care is coordinated and rendered by trained physician intensivists either directly or through directing the care at the helm of multidisciplinary teams. Physician intensivists may act as ICU directors enforcing policies and standardizing care thus providing potential for improved efficiency and patient outcomes (Brilli et al., 2001). Improved outcomes are precisely what distance Closed models from the other two variants. Pollack, Katz, Ruttimann, and Getson, as early as in 1988, retrospectively reviewed pediatric ICU (PICU) records comparing two time periods with and without an intensivist and although no effect on mortality or in length of stay (LOS) was demonstrated the study did show greater use of therapeutic monitoring and improved bed utilization.

The same year, Reynolds et al. (1988) published the results of a retrospective study in which two different time periods were examined for the presence of a critical care-trained faculty in an ICU. Mortality was significantly decreased during the period following the supervision of critical care-trained faculty based on comparisons of severity of illness scores for the two time periods. The following year, a retrospective study conducted in two time periods at a medical ICU (MICU), pre and post the addition of an intensivist was published reporting mortality rates significantly lower during the intensivist time (Brown \& Sullivan, 1989). 
Subsequent studies over the last two decades have found similar results in evidence when evaluating the differences between physician intensivists' staffed units and those not. More recently, Pronovost et al. (1999) published a systematic review of the available literature regarding ICU physician staffing and outcomes and reported findings of decreased mortality and reduced lengths of stay with physician intensivists. Unfortunately, there are no reported randomized, prospective trials comparing the outcomes between various models of ICU staffing and critical care delivery.

The last model of intensive care, Transitional or Semi closed ICUs, are units in where the physician intensivists are present in the unit and available to provide care but the care is shared and co-managed with other physicians like surgeons or internists. The physician intensivists may manage the unit, establish policies and coordinate multidisciplinary efforts but the autonomy in decisions regarding care is a joint venture with other physicians. Although ultimately the decision making about care should rest in the intensivist this stand sometimes generates conflicts that may result in patient detriment and deterioration of peer relations (Brill et al., 2001). In fact, is hard to conceive that a situation that may generate conflict and divergent opinions in care management may allow parties to focus into preserving patient's health and can warrant swift recoveries. Precisely, because of this potential disruption of care, Close ICU besides clearly favor better outcomes remains the most sensible model for care of the critically ill in hospital settings, namely ICUs.

Closed ICUs are synonymous with an intensivist model of critical care delivery and are associated with improved outcomes (less complications, shorter length of stay) and less resource utilization (Nathens et al., 2006). In one systematic meta-analysis of 
published articles reporting both physician staffing and patient outcomes, Pronovost et al. (2002) reviewed 27 studies comprising over 27,000 critically ill adults and children showing Closed ICUs reporting lower hospital mortality, shorter ICU and hospital length of stay. When evaluating the effect of a physician intensivist model of critical care delivery on the risk of death following injury, researchers found that care in a physician intensivist model or Closed ICU was associated with a large reduction in in-hospital mortality following trauma, particularly in the elderly (Nathens et al., 2006). Young and Birkmeyer (2000) also found reductions of mortality rates from $15 \%$ to $60 \%$ in ICUs using physician intensivists.

Despite the available evidence that supports this model on care in ICUs for better patient outcomes only one in three patients in the U.S. is cared for by intensivists, usually in teaching or large hospitals (Angus et al., 2000). Current literature suggests that implementing an intensivist model in critical care settings will help save over 50,000 Americans in the U.S. each year (Young \& Birkmeyer, 2000). If we compound the projected shortage of physician intensivists and other physicians practicing in critical care with the impact of this shortage in smaller urban, rural, and community hospitals in underserved communities, the situation is even more critical, putting vulnerable populations like the elderly at risk (USDHHS, HRSA, 2006).

Understanding the development of ICUs, physician intensivist models of ICU care, and implementation of these models and their impact on the health, economy, and welfare of citizens may facilitate the dissemination of these models. Consequently, the question of whose burden to implement and disseminate such models may lead to explore alternative healthcare options in the delivery of safe, outcome-driven, cost-effective care. 
In a large observational, nonrandomized study using contemporaneous controls Pronovost et al. (1999), using the Maryland Health Discharge Data Set, focused on patients undergoing major abdominal aortic surgery. The study compiled data from 39 of 46 acute care hospitals in the state of Maryland. Using a multi-tiered, multivariate analysis the study showed that that daily rounds in the ICU by an ICU physician (intensivist) was associated with reduced in-hospital mortality and reduced postoperative medical complications. A systematic review was conducted by Pronovost, Young, Dorman, Robinson and Angus (1999) of the available literature on physician staffing and outcomes. The authors concluded that there was a consistent finding of decreased mortality and decreased length of stay with intensivist presence in the ICUs. Although the differences revealed in the study are real and substantiated it is difficult to determine which components of ICU care have resulted in the observed effects. This suggests the need for further studies to compare outcomes of various models of ICUs and determine the components responsible for the improved outcomes.

More recently Gajic et al. (2008) reported the results of a two-year prospective study of patient outcomes, processes of care, and family and provider survey of satisfaction, organization, and culture in the intensive care unit. The effects of 24-hour mandatory critical care specialists (intensivists) in the unit on quality of care, and family and provider satisfaction in the ICU of a teaching hospital was compared to ones with ondemand critical care specialist presence. This is the first study to compare high-intensity (the intensivist is responsible for patient care in a Closed ICU [greater than $80 \%$ of patients]) (Pronovost et al., 2002) intensivist staffing to full-time on-site intensivist coverage. Previous studies have compared process and outcomes of low-intensity 
(elective intensivist consultation, Open ICU, and management of patients by intensivist not mandatory) (Pronovost, 2002) versus high-intensity intensivist staffing.

The introduction of continuous (24 hours) on-site presence of an intensivist was associated with improved processes of care, staff satisfaction, decreased complications, and reduced length of stay. The rate of complications decreased from $11 \%$ to $7 \%$ per patient-day $(\mathrm{p}=.023)$. The introduction of night coverage in the ICU was responsible for these differences and documents the evidence that intensivists' presence in the ICUs is beneficial for patients, families, and overall healthcare (Gajic et al., 2008).

Despite the evidence suggesting the use of high-intensity intensivist staffing to improve patient outcomes and reduce ICU associated costs, $73 \%$ of the ICUs in this country provide low-intensity intensivist staffing or no intensive care coverage at all (Gajic \& Afessa, 2009). Pronovost et al. (2002) analyzing data from 26 studies, including a total of over 14,000 patients with high-intensity intensivist staffing and almost 13, 200 with low-intensity intensivist staffing from over 150 ICUs reported lower mortality rates in 16 studies with 0.71 pooled estimate of the relative risk reduction. ICU and hospital length of stay reduction was also evidenced in most of the studies (Pronovost et al., 2002). Similar findings confirming the association between high-intensity intensivist staffing and good patient outcomes were reported by Pronovost et al. (1999) on abdominal aortic surgery, Dimick, Pronovost, Heitmiller and Lipsett, (2001) on esophageal resection, and Treggiari et al. 2007,on acute lung injury. Reducing the length of stay in the ICU may reduce the morbidity and mortality rates associated with prolonged stay (Pronovost et al., 1999). 
When examining the effects of the implementation of an intensivist model or Closed ICU in light of cost or patient outcomes, the evidence points to the close relationship of these two factors. For instance, in measuring patient outcomes in Closed ICUs, when structural measures were considered ([evaluating quality measurements using Donabedian's three domains: structure, process, and outcomes] staff organization) the evidence suggests lower mortality rates of critically ill surgical patients in Closed ICUs (Birkmeyer, Dimick, \& Birkmeyer, 2004). Hanson et al. (1999) compared two patient cohorts admitted to surgical ICUs (SICUs) during the same period, where the study cohort was supervised by a critical care team lead by an intensivist and the control cohort was cared for by a team supervised by a general surgeon. The patients cared by the team supervised by the intensivist had shorter length of stay in the SICU, used fewer resources, had fewer complications, and had lower hospitalization costs. This is additional supporting evidence of the effectiveness of this model on patient outcomes and delivery of healthcare services aimed to improve patient care.

Although the extensive published literature supports the intensivist model of Closed ICUs most of these studies were retrospective and all were observational. The major limitation of published studies focusing in the impact of ICU intensivist staffing on patient outcome has been the lack of randomized prospective designs (Gajic \& Afessa, 2009). The ethical considerations behind a randomized clinical trial that will assign patients to trained intensivists versus not trained physicians preclude such study.

In addition, a study published in 2008 by Levy and colleagues indicated that highintensity ICU physician staffing had higher severity-adjusted mortality compared to lowintensity ICUs. However, the study was based on retrospective analyses of data collected 
for other purposes, thus failing to provide possible explanations regarding the conflicting results. Also, critically ill patients with higher severity of illness were less likely to be admitted to ICUs with no access to intensivists' care which may have resulted in a design bias.

Over 5 billion dollars a year could be saved in healthcare costs by implementing physician intensivist staffing in non-rural U.S. hospitals (Pronovost et al., 1999). However, the shortage of intensivists and failure to implement the Accreditation Commission of Colleges on Medicine (ACCM) guidelines for ICU staffing may prevent not only saving these healthcare costs but lives as well. Therefore, new ICU staffing paradigms are needed to provide safe, cost-effective, and suitable care to critically ill patients.

\section{Advanced Practice Nurse (APN) Intensivist Services}

While several studies have documented positive outcomes for the physician intensivist model (Hanson et al., 1999; Pronovost et al., 1999; Pronovost et al., 2002; Reynolds et al., 1988) in ICUs, very few have studied the role of APNs as intensivists in the management of critically ill ICU patients. Durbin (2006), in advocating for care in ICUs through an intensivist-lead Team Model, pointed to an estimated 30\% - 50\% reduction in mortality when intensivists rounded daily on critically ill patients, and to the shortening of the length of stay, reduction in cost, and prevention of complications with the implementation of this model. However, Durbin failed to acknowledge the possible role of APN intensivists in the care of the critically ill patients and their effect on patient outcomes. 
APNs, also referred to as mid-level practitioners or non-physician providers, are nurses practicing at an advanced clinical level. The APN designation includes Clinical Nurse Specialists (CNSs), Nurse Practitioners (NPs), Nurse Midwives (NM), and Nurse Anesthetists (NAs,) (Sheer \& Wong, 2008). Internationally, the title APN includes nurses prepared as Clinical Nurse Specialist, Nurse Practitioner, Certified Nurse Midwife (CNMW), and Certified Registered Nurse Anesthetist (CRNA). APN roles have evolved in clinical specialty areas including Community Health Nurse Practitioner (CHNP), Neonatal Nurse Practitioner (NNP), Pediatric Nurse Practitioner (PNP), Family Nurse Practitioner (FNP), Acute Care Nurse Practitioner (ACNP) and most recently, APN Intensivist. Worldwide, APN practice has developed and became a global healthcare trend over the last decades (Sheer \& Wong, 2008; Brooten et al., 2011).

The role and use of advanced practice nurses (APNs) has expanded globally due to increased needs for health care providers with advanced knowledge and skills (Sheer \& Wong, 2008; Brooten et al., 2011). The International Council of Nurses (2008) defines the Nurse Practitioner/Advanced Practice Nurse as a registered nurse who has acquired the expert knowledge base, complex decision-making skills and clinical competencies for expanded practice. The characteristics of which are shaped by the context and/or country in which he or she is credentialed to practice. A master's degree is recommended for entry.

Nurse Practitioners (NPs) are licensed independent practitioners prepared to provide services across the health wellness-illness continuum for their specialty population. NPs practice focuses on direct patient care. They have acquired an advanced level of competencies, skills and autonomy through training and clinical practice (APRN 
Joint Dialogue Group, 2008). More than $88 \%$ have completed graduate-level education and $93 \%$ have obtained certification from a national certification program (U.S. Department of Health \& Human Services, Health Resources \& Services Administration. [USDHHS, HRSA], 2010).

In contrast to the attrition of physician intensivists and critical care trained physicians the number of NPs is increasing in acute care specialties, both in the United States and globally. From approximately 97,000 registered NPs in the U.S. in 2004 (Goolsby, 2005) their numbers have increased to almost 160,000 in 2008 (USDHHS, HRSA, 2010). The NP presence in the healthcare workforce is growing. Although only $5.8 \%$ of the total practicing NPs are employed in critical care (USDHHS, HRSA) this number continues to increase as more university graduate programs expand to include acute care tracks in their core curricula. However, despite their increased visibility and participation in the healthcare arena, healthcare providers and potential employers are still unfamiliar with the scope of practice of APNs (McLaughlin, 2007).

Several hundred studies have been conducted examining APN practices including outcomes of APN practices in primary care compared to physician practices (Mundinger, 2000), acute care nurse practitioner (ACNP) hospital managed care compared to that of physicians (Kleinpell \& Gawlinski, 2005) and research examining effects of APNs on patient outcomes and health care costs in vulnerable, high volume or high cost patient groups (Brooten et al., 2002). Other research focused on the APN role, components of the role, time spent in each role component, and perceptions of the role by various stakeholders in health care. Research on the effects of APN intensivists is only beginning. The following section examines the history of APNs in ICU Care, current 
APN care in ICUs, and research on effects of APN care in ICUs on patient outcomes and health care costs.

History of APNs in Acute Care. Historically, the role of APNs evolved due to the advancement of care and the decreased number of physicians available. Advanced Practice Nurses (APNs), have been called mid-level practitioners, physician substitutes, or non-physician providers, nurses practicing at an advanced clinical level and required to have advanced nursing education beyond Registered Nurse licensure. The Nurse Practitioner (NP) role was initially developed in pediatrics in 1965 as a combined nursephysician effort at the University of Colorado. The role was designed in response to the unavailability of adequate numbers of physicians to provide the needed care for children (Bupert, 2004, p 4-6).

The focus of preparing NPs was to enhance patient access to care. Nurse Practitioner training is supported by federal and state funding and thus is viewed to be a government sponsored asset (Starck, 2005). Nurse Practitioner training is faster and less expensive compared to physician training. Three to 12 NPs can be educated for the cost of educating one physician (Starck, 2005). When the setting of NP practice transitioned from primary to the acute care setting different educational preparation was required (Page \& Arena, 1994).

Nurse Practitioners are registered professional nurses prepared through advance education and clinical training to deliver preventative and acute health care services to individuals across the lifespan (American College of Nurse Practitioners, n.d.-a). The NP educational model is based on content that includes clinical medicine, psychology, sociology, and education (Bullough, 1992). They have advanced education in 
pathophysiology, pharmacology, clinical diagnosis, and treatment allowing them to diagnose and prescribe treatments in their specialty area (American Academy of Nurse Practitioners [AANP], 2010c). They are licensed independent practitioners practicing in ambulatory, acute, and long term care as primary or specialty care providers (American Academy of Nurse Practitioners [AANP], 2010b).

Nurse Practitioners have been providing quality care since 1965 and they provide comprehensive care within an area of specialization (American College of Nurse Practitioners, n.d.-b). Nurse Practitioners practice allows for them to evaluate, diagnose, manage healthcare problems, promote health and collaborate with patients, families, and healthcare providers (American College of Nurse Practitioners, n.d.-b). The state's nurse practice act regulates NPs practice and therefore it varies from state-to-state. Some states allow for independent practice (not requiring physician involvement) and some require collaborative agreements with a physician to practice (American College of Nurse Practitioners, n.d.-b).

Nurse Practitioners, in general, serve as primary or secondary providers of care in either primary or specialty care settings. They provide patients and families with a care modality that combines nursing and medical practices and processes, patient education, and other healthcare services in an effort to optimize patients' health and healthcare delivery. This extended range of care is what makes the NP role so unique and valuable.

Nurse Practitioners are prepared to provide services across the health wellnessillness continuum to the population in their specialty. Nurse Practitioners practice in direct patient care and have acquired an advanced level of competencies, skills, and autonomy through training and clinical practice (APRN Joint Dialogue Group, 2008). In 
2004 there were approximately 97,000 NPs registered in the U.S. (Goolsby, 2005). This number grew to almost 160,000 in 2008 (USDHHS, HRSA, 2010), and their numbers continue to increase and their roles to expand.

Nurse Practitioners, as primary care providers, may be reimbursed under Medicare, Medicaid, and by private insurers or impaneled as primary care providers by Healthcare Maintenance Organizations (HMOs) (American College of Nurse Practitioners, n.d.-b). The prescriptive authority of NPs is essential to their practice. The ability of NPs to prescribe without limitation is essential to provide cost-effective, quality health care for the diverse populations they serve (AANP, 2010c).

During the 1970s, the NP role was developed to focus on the role of NPs in the neonatal acute care setting. Approximately two decades later, the National Association of Neonatal Nurses published Guidelines for Neonatal Nurse Practitioners' Education Programs to standardize the curriculum and certification of the Neonatal Nurse Practitioners. The NP role started emerging in other specialties; however, the history of the beginning of the role in other specialties such as acute and critical care is not well documented.

APNs in Critical and ICU Care. In 1995 the role of the Acute Care Nurse Practitioner (ACNP) was developed when the American Association of Critical Care Nurses and the American Nursing Association released the Standards of Clinical Practice and Scope of Practice for the Acute Care Nurse Practitioner (Cramer, Orlowski, \& DeNicola, 2008). Acute Care Nurse Practitioners represent slightly over five percent of the 135,000 NPs practicing in the U.S., with an average of seven years of practice in acute care settings (American Academy of Nurse Practitioners [AANP], 2010a). The 
evolution of the role of ACNPs in acute care has responded to an increased need of tertiary services, the shortage of medical staffing to respond to these needs, and the advancement of the role of ACNPs in clinical practice. In intensive care units (ICUs), the role of ACNPs is evolving to replace physicians and support patient care rather than providing the traditional holistic nursing care (Knaus et al., 1997; Mundinger, 1999). As a result ACNP practice in ICUs follows a medical model of care (Irvine et al., 2000). This contrast to the more traditional role of nurses and APNs led to studies that compared the roles of CNSs and ACNPs in different settings and especially in critical care.

Clinical Nurse Specialists (CNSs) and Nurse Practitioners (NPs) continue to be the predominant APNs in critical care in the U.S. (Coombs, Chaboyer, \& Sole, 2007). The differences in role definition and scope of practice of these two types of APNs are stipulated by their licensure and the governing state laws. In critical care, CNSs are more involved in case management, indirect care, and education. Acute Care Nurse Practitioners (ACNPs) are more involved providing direct care (Coombs et al., 2007) which generates revenues (Cramer et al., 2008). Subsequently, several comparative studies investigating these differences in roles (Ackerman, Norsen, Martin, Wiedrich \& Kitzman, 1996; Becker, Kaplow, Muenzen, \& Hartigan, 2006; Kleinpell, 1998; Coombs et al., 2007; Mick \& Ackerman, 2000) have been published.

In the early 1990s, the acute care nurse practitioner role was relatively new and not yet clearly defined. Ackerman et al. (1996) explored the evolution of the Clinical Nurse Specialist role into the ACNP role by identifying domains of practice common to both roles (i.e., direct comprehensive care, support of systems, education, professional leadership, research and publication). As a result, the authors developed the Strong 
Model of Advanced Practice that included the conceptual strands of empowerment, collaboration, and scholarship. The model was developed by conducting an intensive literature review, expert consultation, brainstorming, and a review of practice. A continuum of experience from novice to expert was also identified within each domain. The authors concluded that the model was accurate but still in need of further testing (Ackerman et al., 1996). This model was intended to guide institutions and individual practitioners in role definition and scope of practice.

Mick and Ackerman (2000) tested the Strong Model in a descriptive exploratory study with 18 APNs (6 CNSs and 12 NPs). The study questionnaires included selfranking of expertise in practice domains and valuing role related tasks. The findings revealed that CNSs self-ranked their expertise higher in all practice domains by giving greater emphasis on education, research and leadership. ACNPs placed higher value on tasks related to direct patient care, diagnosing, and performing diagnostic procedures (Mick \& Ackerman, 2000). These findings emphasize the role differences between CNSs and ACNPs in scope of practice, roles, and responsibilities (Coombs et al., 2007). Divergent levels of emphasis on collaboration versus autonomy also differentiate the scope of practice between CNSs and ACNPs (Mick \& Ackerman, 2000). ACNPs were found to emphasize direct comprehensive care tasks and CNSs were involved with education, research, and leadership.

The CNS performs intradisciplinary and interdisciplinary consultative and collaborative skills in practice, while NPs concentrate on developing unit-or servicebased professional autonomy in a collaborative practice relationship with physicians (Mick \& Ackerman, 2000). Study findings were limited by the small sample size and 
inclusion of nurses from different sites and countries where the scope of practice, roles, responsibilities, and healthcare models might vary. Including objective measures in addition to the self to compare these two roles may have strengthened study findings. In differentiating these roles, Kleinpell (1998) acknowledged that the focus of ACNPs is on specialized knowledge and skill in the management of select patient groups with acute and specialized needs. By merging role aspects of CNSs and NPs, the ACNPs role evolved into expanded practice caring for acute and critically ill patients in areas like ICUs (Ackerman, 1997; Coombs et al., 2007).

Cajulis \& Fitzpatrick (2007) designed a descriptive study to determine the level of autonomy of NPs in an acute care setting, trained as adult nurse practitioners (ANPs), ACNPs, geriatric nurse practitioners (GNPs), and family nurse practitioners (FNPs). The study was conducted in a large metropolitan hospital and the sample population was NPs practicing with adults inpatients $(\mathrm{n}=54)$. They used The Dempster Practice Behavioral Scale (DBPS) to measure the level of autonomy of NPs. Descriptive statistics were used for demographics and DBPS scores, and Pearson correlation coefficient was used to test relationship between autonomy scores and demographic variables. Resulting data indicated that the majority of NPs had high levels of autonomy, competence, skills, and mastery. Around $50 \%$ of NPs had very high levels, $31.5 \%$ had extremely high levels, and $19 \%$ had moderate levels of autonomy. Data revealed that the majority of the NPs were highly empowered, accountable, and responsible in providing care in acute care settings (Cajulis \& Fitzpatrick, 2007).

In addition to ACNPs some CNS are still practicing in the ICUs functioning as liaisons to patients and families, and in managerial roles (National Association of Clinical 
Nurse Specialist [NACS], n.d.). Some NPs, particularly Adult Care NPs trained in critical care also practice in ICUs by virtue of competency and training, and grandfathering in the role. Today ACNPs, especially in the practice of critical care in ICUs, have evolved into sophisticated and highly skilled practitioners blending the paradigms of nursing practice with the therapeutic paradigm of medicine. An example of this trend is the inception of virtual care interventions through the use of telemedicine. The heavy demand for critical care services in the U.S. is growing; almost 55,000 patients receive care daily in approximately 6,000 ICUs registered in the nation (SCCM, 2006). Acute Care Nurse Practitioners are finding the need to diversify and enhance their practice in response to these healthcare needs (Amba, 2011).

This need for ACNPs to diversify and enhance their practice is resulting in them seeking certification and training in sub-specialty areas such as intensivists in ICUs. Several published studies support the adequacy and competency of ACNPs in critical care, but research is needed to evaluate the effect of the ACNPs as intensivists on patient outcomes and cost effectiveness.

Becker and colleagues (2006) described the activities of ACNPs and CNSs for the purpose of examining nurse competencies for certification. In a sample of $158 \mathrm{CNSs}$ and 77 ACNPs, $85 \%$ of ACNPs worked in the role of NPs in acute care while the CNSs were reported to hold positions that varied between the CNS role $(72 \%)$, educators $(9 \%)$, as first-line managers, middle managers, and nurse researchers (3-4\%). Half of the CNSs $(50 \%)$ worked in nonprofit community hospitals compared to $26 \%$ of ACNPs. In this sample, CNSs were twice as likely as ACNPs to work in ICU settings. ACNPs were more than twice as likely as CNSs to work in specialized or other acute care units (e.g., 
burn and cardiology units). The findings of this study supported Kleinpell's (2005)

results where ACNPs spent most of their time (73-76\%) providing direct care (Becker, et al, 2006). Despite the national significance of the study the response rate was low (35\%).

Subsequent studies emerged examining the role of ACNPs. These studies continued to compare the role of the ACNP with CNSs in addition to studying the different models of care and their effects on patient and organizational outcomes (BryantLukosius, DiCenso, Browne, \& Pinelli, 2004). With the growth of the ACNP role, training and educational needs arose and regulatory considerations were determined.

Acute Care Nurse Practitioners in Critical and ICU Care. ACNPs function as members of multidisciplinary teams in closed (intensivist lead) or semi-closed (intensivist shared lead) ICUs. Collaborative practice rather than individual or autonomous practice is the norm in both instances. However, Acute Care Nurse Practitioners still enjoy autonomy in decision making and treatment modalities as permitted by the scope of their respective collaborative agreements. Studies indicated the $47 \%$ of ACNPs work in urban settings and $45 \%$ in teaching hospitals in northeastern states in the U.S. The ACNP responsibilities included care coordination (98\%), diagnostic testing (97\%), initiating specialty consultation (91\%), and initiating discharge planning (84\%) (Kleinpell, 1997).

Acute Care Nurse Practitioners (ACNPs) in critical care areas are trained to assume their role under the supervision of an attending physician. The ACNP certification has been available since 1995 (Kleinpell, 1999). The initiation of certification led to the development of educational programs that specifically prepare ACNPs for the role of intensivists in the ICU (Raines, 2008; Rivers, 2010). The designation of ACNP can be used by those who graduated and are certified as an ACNP. 
However, any specialty-trained NP can work in acute care (Melander, Kleinpell, \& McLaughlin, 2007).

Education, clinical training, and skills acquisition continues to be of absolute importance in the making of expert clinicians. In one study, a skills questionnaire was developed based on literature review, evaluation of scope and standards of Acute Care Nurse Practitioners (ACNPs) practice, and ACNPs role descriptions (Kleinpell, Hravnak, Werner, \& Guzman, 2006), and sent to program directors of ACNP programs in U.S. $(n=71)$ to assess the type of skills currently taught in their program. The results revealed that most ACNP programs in the U.S. are adequate in depth and breadth in preparing ACNPs for critical care. Although the authors identified a lack of specialty-based skills being taught (only $5 \%$ of the programs), the authors noted that these skills are usually acquired through training (Kleinpell et al., 2006). However, the researchers recommended incorporating and increasing simulation technology to stimulate critical decision making and clinical skills in lieu of specialty-based skill training.

Kleinpell (2005), in the largest study of ACNP practice to date, reported the cumulative results of a national 5-year longitudinal survey of ACNP practice. Beginning in 1996, Kleinpell conducted a series of surveys of nurse practitioners seeking national certification as ACNPs. These surveys examined the role aspects, practice components and role changes after certification as an ACNP. At the time of the publication more than 3,500 advanced practice nurses were certified as ACNPs. The data were collected using a 44 question survey that included items that assessed role aspects, practice components, and role changes after certification including items on practice settings, role responsibilities, and aspects of practice. The survey included items about credentialing 
and privileging status, frequently performed procedures, work requirements, role changes, and plans for employment. These surveys were mailed annually to the same group of nurses with additional questions about the ACNP's practice in aspects such as negotiation of benefits, recommendations for practitioners, recommendations for educators, and outcome evaluation. The study revealed that the majority of ACNPs practiced in ICUs and specialty acute care tertiary settings (emergency, trauma, urgent care). These results illustrate the diverse practice settings of ACNPs. The 437 participants revealed that $85-88 \%$ of the time ACNPs focus in direct management of patient care such as performing physical examination, writing orders, interpreting laboratory and diagnostic tests, performing procedures, and discharge planning. Participants self-reported their impact on outcomes in terms of decreased length of stay, healthcare cost, readmission rates, complications, and resources utilization. They also increased continuity of care, patient satisfaction and education, and patient access to care. During the study period, ACNP roles evolved within their practice. Specialty based practice increased from $37 \%$ in year 1 to $49.5 \%$ in year 5 and collaborative practice roles increased from $17 \%$ to $25 \%$ in years 1 and 5 respectively (Kleinpell, 2005). The results of this study were useful to physician collaborators and administrators in understanding the role of ACNPs. However, the longitudinal nature of the study led to the attrition of participants during the five years of data collection. Also, ACNP representation was limited to the West, Pacific, and South Central regions of the U.S. (Kleinpell, 2005).

Kleinpell and Goolsby (2006) also reported results of an NP survey that focused on acute care for the American Academy of Nurse Practitioners. Of over 16,000 NP respondents, 685 self-reported acute care as their specialty. A variety of specialty areas 
of ACNP practice were self-reported, including cardiovascular, pulmonary, neurology, gastroenterology, orthopedics, hematology/oncology, palliative/pain), nephrology, urology, infectious disease, ear, nose, and throat, rheumatology, sports medicine and others. A majority of ACNP respondents reported practicing in an inpatient hospital setting, $(55.7 \%, \mathrm{n}=365)$, followed by physician practice, $(15.4 \%, \mathrm{n}=101)$ or hospital outpatient setting $(9.9 \%, \mathrm{n}=65)$. The findings of this study helped define the specific roles and responsibilities of NPs practicing in acute care (Kleinpell \& Goolsby, 2006).

Although ICU and specialty acute care tertiary settings are areas where a significant number of ACNPs practice, the Kleinpell study (2005) showed an increase in practice settings not limited to ICUs (e.g. transplant services, pre-surgical and preanesthesia, oncology) (Kleinpell, 2005). While management of patient care remains the primary responsibility of ACNPs, the survey also revealed evolution into other roles of the ACNPs different from direct care.

Advanced Practice Nurse Intensivist. The role of APNs as intensivists is one that merits research on the effects of APN Intensivists on patient outcomes, quality of care, and costs associated with this practice modality. The APN Intensivist is a healthcare provider with a high level of independence who specializes in the management of critically ill patients in critical care settings. A masters or doctoral degree is required (Vanderbilt University, 2007) as well as national certification in advanced nursing practice (i.e., Acute Care Nurse Practitioner). They usually practice as part of multidisciplinary intensive care teams.

Very little has been published on this relatively new sub-specialty role of Acute Care Nurse Practitioners (ACNPs). A systematic search of databases: Medline, Google 
scholar, and full-text CINAHL, using the words "Acute Care Nurse Practitioner Intensivists", "ACNP intensivists", "APN intensivist", and "NP intensivist", yielded only one article where the role of ACNPs as intensivist (pediatric intensivist extenders) was implicit (Cramer et al., 2008). Another was an abstract on ACNPs roles in a surgical ICU (Wyckoff \& Kaliff, 2009). The abstract attempted to associate the terms ACNPs and intensivists with an intensivist theory but failed to demonstrate any direct relationship or role delineation for the ACNP as intensivists, or a theory to explain this practice role. The lack of literature examining the role of ACNPs as intensivists and of research to evaluate the effectiveness of this role makes clear the need for further research to examine the effects of the role on healthcare costs, on quality of care and patient outcomes.

In examining education and preparation for this sub-specialty role, inquiry into multiple databases yielded one university program at the graduate level (Vanderbilt University) and another at a hospital-based training (Emory University). Both are in the U.S. At Vanderbilt, ACNP intensivist students are prepared to practice on multidisciplinary intensive care teams in tertiary care centers. This subspecialty focuses on providing care for critically ill patients such as those with cardiac arrest, shock, respiratory failure, multiple organ failure, sepsis, stroke, and brain injury.

The focus of the ACNP Intensivist track is to provide clinical experiences and pathophysiological background for graduates and to prepare them to join critical care intensivist teams. Vanderbilt's program pairs Nursing ACNP faculty with Critical Care physicians to provide advanced critical care didactic content, ICU clinical rotations, and highly sophisticated critical care simulations. Students rotate through five intensive care 
units including surgical ICU (SICU), Medical ICU (MICU), Neurology ICU (Neuro ICU), Cardiovascular ICU (CVICU) and the Burn Unit. Within each rotation students are mentored in diagnosing and managing complex patients and guided in the development and refinement of their own clinical skills (Vanderbilt University, 2007).

At Emory, the training follows a different model by being hospital based. The neurology ICU at Emory Healthcare has developed a model that uses ACNPs as intensivists. The practice has grown from one ACNP intensivist to nine. The ACNP does daily rounds on patients and provides advanced care, which may include inserting arterial lines, performing lumbar punctures, or endotracheal intubations. Although the author mentions that research has been done in the unit showing that patients followed by an intensivist had a decreased length of stay in the unit and fewer complications no data has been published from these initiatives to date (Raines, 2008).

In summary, the empirical evidence of the effect of APN Intensivists on patient outcomes remains scarce compared to the well documented effects of APNs in other areas and models of healthcare delivery. In light of this dearth of the literature examining the effects of APN Intensivists on patient outcomes, is needed to fill this gap in our knowledge.

\section{Patient Outcomes}

Understanding patient outcomes or the resulting effect of healthcare interventions may facilitate defining treatment objectives, the scope of interventions, and the resources needed to accomplish best outcomes. It is almost impossible to talk about patient outcomes without linking them to healthcare costs and vice versa. This phenomenon may be closely related to current practice trends in healthcare and healthcare reforms calling 
for a more cost efficient healthcare delivery and optimization of patients' outcomes (American Hospital Association, 2010). In the field of critical care, especially in supporting the need to implement standardized care models for ICUs, research findings are well documented. Patient conditions that are costly in human and economic terms include HAIs (i.e., VAP, CAUTI), pressure ulcers, patient-initiated accidental removal of devices, post-surgical glycemic control, reduction of days on the ventilator and days with indwelling bladder catheter, and ventilator weaning.

Healthcare-Associated Infections (HAIs). The incidence of Healthcare Associated Infections (HAIs) incidence continues to rise resulting in increased mortality and morbidity (Combes et al., 2004; Kieninger \& Lipsett, 2009; Keoning \& Truwit, 2006). The need for a standardized definition of HAIs was addressed by the Centers for Disease Control (CDC) and Prevention in 2005 and subsequently by other professional organizations (CDC, 2005). The National Healthcare Safety Network (NHSN), Division of Healthcare Quality Promotion at the CDC defined HAIs for the purpose of surveillance in the acute care setting as a localized or systemic condition resulting from an adverse reaction to the presence of an infectious agent(s) or its toxin(s) (Horan et al., 2008). The infection was not present or incubating at the time of admission to the acute care setting (Horan et al., 2008). Infections associated with complications from existing infections at admission time, colonization (presence of microorganisms without adverse effects, symptoms, or clinical signs of infection), and inflammation from tissue response to injury or to noninfectious agents (e.g., chemicals) are excluded (Horan et al., 2008).

HAIs may be classified as either endogenous or exogenous (Horan et al., 2008). Endogenous sources are microorganisms that normally inhabit certain body sites, as in 
gastrointestinal flora. Exogenous sources are considered those microorganisms that are usually transmitted to patients from external sources in the healthcare environment such as medical devices, patient care personnel, visitors, and patient equipment (Horan et al., 2008). Once a diagnosis of HAI is made, the specific type of infection is identified.

The long term effects of HAIs have not been discussed in the literature. The majority of the published reports and studies to date focus on preventive measures (Drakulovic et al., 1999; Elpern et al., 2009; Grap et al., 2003; Munro et al., 2009; Shahin, Dassen, \& Halfens, 2009), diagnostic procedures (Kollef et al., 2008; Sole et al., 2002), mortality (Kieninger \& Lipsett, 2009; Keoning \& Truwit, 2006), morbidity (Combes et al., 2004), length of stay (Pyenson et al., 2007; Shorr, Combes, Kollef, \& Chastre, 2006; Williams et al., 2010), and cost (Combes et al., 2004; Maki \& Tambyah, 2001; Safdar, Dezfulian, Collard, \& Saint, 2005; Scott, 2009). HAIs are a resulting complication of multifactor events associated with healthcare personnel and healthcare circumstances. The resulting extended hospitalization may lead to extended costs (e.g., additional laboratory tests, diagnostic procedures, and interventions).

The aim of the medical management of HAIs is curative in nature. In the absence of cure or resolution of the complication, patients may remain hospitalized longer thus increasing morbidity and mortality and preventing them from being discharged to lower acuity settings. Perhaps the delay in the discharge process of hospitalized patients with HAIs waiting for infection resolution may explain why most of these studies focus on the reduction of length of stay and cost in the ICU. The lack of long term studies may also be explained by understanding that HAIs are either resolved in the ICU or patients die as a consequence of the infection. 
Ventilator-Associated Pneumonia (VAP). Healthcare-associated pneumonias, specifically VAP, are seen in patients admitted to critical care units, requiring mechanical ventilation, and ending with a prolonged length of stay. VAP is an HAI developed by persons using a mechanical ventilator to assist or control respiration continuously through either a tracheostomy or an endotracheal tube within 48 hours of intubation (early onset) or during the weaning period (late onset) (Horan et al., 2008).

VAP, accounting for $15 \%$ of all HAIs, is frequently lethal, contributes greatly to increased health care costs, and accounts for higher indexes of morbidity and mortality (Kieninger \& Lipsett, 2009). Eighty six percent of healthcare-associated pneumonias are associated with mechanical ventilation (Koenig \& Truwit, 2006). Mortality rates range from $10 \%-70 \%$ increasing the likelihood of death 3-to-4 fold, resulting in extended hospitalizations, excess use of antimicrobials, and increased direct medical costs by up to $\$ 30,000$ per case (Coffin et al., 2008; Sole et al., 2002). In fact VAP is a leading cause of death in critically ill patients (Eachempati et al., 2009). Recent studies report rates of VAP from 1 to 4 cases per 1,000 ventilator-days, although it may exceed 10 cases in high risk populations such as surgical ICU patients (Coffin et al., 2008). VAP accounts for 250,000 to 300,000 cases per year in the U.S. alone (Koenig \& Truwit, 2006).

The pathogenesis of VAP is multifactorial (Kieninger \& Lipsett, 2009). Invasion of pulmonary tissue in patients with VAP typically arises from aspiration of secretions, colonization of the tract (i.e., biofilm), and use of contaminated equipment (Coffin et al., 2008). Diagnosing VAP is usually a combination of high clinical suspicion, positive physical examination findings, radiographic signs indicative of pneumonia, and confirmatory laboratory findings (Koenig \& Truwit, 2006). New or progressive 
infiltrates on chest radiographs, increased white blood count (leukocytosis), and purulent tracheobronchial secretions are highly indicative of VAP (Koenig \& Truwit, 2006).

Targeting modifiable risk factors can decrease rates of postoperative pneumonia. Minimizing the risk of aspiration by elevating the head of the bed at least 30 degrees if feasible, is recommended (American Thoracic Society [ATS], 2005; Kieninger \& Lipsett, 2009; Metheny et al., 2006). Also elimination of colonizing organisms in the upper aerodigestive tract by providing good oral hygiene and the use of chlorhexidine gluconate rinse may prove beneficial in reducing the incidence of VAP (Kieninger \& Lipsett, 2009; Segers, Speekenbrink, Ubbink, van Ogtrop, \& de Mol, 2006). In addition to the decontamination of the aerodigestive tract, stress ulcer prophylaxis may decrease morbidity and mortality. ICU patients are at an increased risk for stress ulceration resulting in gastrointestinal bleeding episodes that may increase their mortality (Cook et al., 2001). For these reasons the use of histamine blockers and proton pump inhibitors is indicated to reduce the incidence of peptic ulcerations in mechanical ventilated patients (Dodek et al., 2004).

Another target for risk reduction is the management of the mechanical airway system including the endotracheal tube and the ventilator circuit. The presence of an endotracheal tube (ETT) allows for biofilm formation thus promoting the adherence of bacteria to the biofilm contributing to the colonization of the airway and impeding antibiotic penetrance (Kieninger \& Lipsett, 2009). The elimination of secretions pooling on the ETT cuff and the consequent reduction of tracheal contamination has been successfully demonstrated in several clinical trials, hence the current evidence-based guidelines advocating for the use of subglottic secretion drainage through the use of ETT 
with subglottic suction port (Kieninger \& Lipsett, 2009; Sole et al., 2002). Risk factors associated with VAP include prolonged intubation, enteral feeding administration (e.g., nasogastric, small bowel feeding, or gastrostomy tubes), witnessed aspiration, use of paralytic agents, underlying illness and extremes of age (Coffin et al., 2008).

In a randomized control trial, Drakulovic et al. (1999) reported the results of a study that examined supine position as a risk factor for VAP. Patients were recruited from one medical and one respiratory ICU and randomized into semi recumbent $(n=39)$ or supine ( $\mathrm{n}=47$ ) body positions. Data were collected on the frequency of clinically suspected and microbiologically confirmed VAP in both groups. The frequency of clinically and microbiologically confirmed suspected VAP was significantly lower in the semi recumbent group than the supine group. The findings of this study were the beginning of several consequent studies that examined position as a risk factor of VAP. The limitations of this study included the lack of a strict control of 45 degrees bed angle in the semi recumbent group and therefore the exclusion of three patients due to protocol violation. In addition, enteral feeding was a confounding factor that increased the incidence of VAP in the control group and contributed to the significant differences observed in the study (Drakulovic et al., 1999).

Van Nieuwenhoven and colleagues (2006), in a multicenter prospective trial of patients in the ICU, assessed the feasibility of the semi recumbent position in mechanically ventilated patients $(n=221)$ in the ICU and determined the effectiveness of an intervention to prevent microbiologically proven VAP. Patients were randomized into semi recumbent or supine position. Mean back-rest elevation was measured every minute using an ingenious system planted in the bed frame. The findings showed that the 
targeted 45 degrees bed elevation in the semi recumbent position was not achieved in $85 \%$ of the study time in the intervention group (van Nieuwenhoven et al., 2006). The importance of this study is that there were no significant differences in control and intervention groups in regards to microbiologically proven VAP. Despite the limitations of being unable to maintain the targeted bed elevation, the sample size was larger and the diagnosis of VAP was microbiologically proven contrary to the previously discussed Drakulovic et al. (1999) study that used clinical criteria and no quantitative cultures. Despite strong evidence suggesting that supine position is detrimental to patients on mechanical ventilation, no conclusive evidence points to a recommendation regarding the angle of semirecumbency needed to achieve statistical significance.

Grap et al. (2003) also conducted a descriptive study to document the level of backrest elevation and identify factors associated with patient positioning in a medical, surgical, and neuroscience intensive care unit. In a sample of 170 adults, backrest elevation was determined by electronic bed read-out (i.e., bed frame elevation gauge). Data on blood pressure, heart rate, and enteral feeding status were also collected. The mean backrest elevation was 19.2 degrees and $70 \%$ of the patients were in supine position. The findings showed no difference in the backrest elevation among units or by enteral feeding status. There were significant correlations between backrest elevation and blood pressure (Grap et al., 2003). The results suggested that the use of higher backrest position in critically ill patients to minimize the risk of aspiration and the incidence of VAP with minimal effect on patient hemodynamics.

Subsequently, Grap and colleagues (2005) described the relationship between backrest elevation and development of VAP in a nonexperimental descriptive 
longitudinal study. The Clinical Pulmonary Infection Score (CPIS) (a proxy for pneumonia) was used to identify VAP within 24 hours of admission to the study (i.e., upon intubation); at 72 and 96 hours (i.e., during day 4 of intubation); and at 144 and 168 hours after intubation (i.e., during day 7 of mechanical ventilation). Backrest was measured continuously using a transducer system in addition to continuous measurement of heart rate and blood pressure from the time of admission to the study for 7 days. Sixty six patients (276 patient days) were monitored. The findings showed that the mean backrest elevation was 21.7 degrees. On day 4, 37 patients remained in the study and $26 \%$ had CPIS indicating VAP. On day 7, 16 patients remained in the study and 31\% had CPIS indicating VAP. The CPIS score increased but was not statistically significant. Backrest elevation had no direct effect on CPIS. Regression analysis to determine the predictors of VAP by CPIS on day 4 documented that baseline CPIS, percentage of time spent at less than 30 degrees elevation on day 1, and Acute Physiology and Chronic Health Evaluation (APACHE) II score contributed to the model with $81 \%$ variability ( $\mathrm{F}=$ $7.31, \mathrm{p}=.003)($ Grap et al., 2005).

Sole et al. (2002) identified the pathogens associated with VAP in oral and endotracheal aspirates and evaluated the bacterial growth on oral and endotracheal suctioning equipment. A prospective descriptive study was conducted on 20 patients orally intubated for at least 24 hours in medical, neurosurgical, and surgical-trauma intensive care units. Baseline samples of oral secretions and endotracheal aspirates were obtained at baseline and after 24 and 48 hours. Suctioning equipment was changed at baseline and remained during the data collection period. The findings showed that after 24 hours all patients had potential pathogens in the mouth and $67 \%$ had sputum cultures 
positive for pathogens. Suctioning devices were colonized with many of the same pathogens that were present in the mouth. The majority (94\%) of tonsil suction devices were colonized within 24 hours. The presence of pathogens in oral and sputum specimens in the majority of the sample supports the premise that micro aspiration of secretions occurs and VAP is a subsequent diagnosis. The equipment used for oral and endotracheal suctioning showed colonization within 24 hours and therefore reusable oral suction equipment contributes to colonization and VAP (Sole et al., 2002). The findings of this study identified nursing interventions (i.e., strict adherence to infection control practices, adequate oral care, and suctioning techniques) that contribute to the decontamination of the oral cavity and the decrease in VAP incidence.

Bouza and colleagues (2008) compared the conventional and continuous aspiration of subglottic secretion procedures in ventilated patients after major heart surgery in a 2-year randomized comparison study. On admission to the ICU, patients were randomized into the continuous aspiration of subglottic secretions group $(\mathrm{n}=359)$ and the conventional care group $(\mathrm{n}=331)$. The conventional care included tracheal aspiration through the lumen of the endotracheal tube as needed by nurses. Cuff pressures were maintained between 20 and $30 \mathrm{~mm} \mathrm{Hg}$. The patients in the continuous aspiration of subglottic secretions group were connected to a continuous system of subglottic aspiration with negative pressure between 100 and $150 \mathrm{~mm} \mathrm{Hg}$. Once per shift, the cuff pressure was checked and $10 \mathrm{~mL}$ of distilled water was added through the subglottic lumen to keep it patent. The incidence of VAP in the intervention and control groups was 3.6\% and 3.8\% respectively. In patients with mechanical ventilation for more than 48 hours VAP incidences were $26.7 \%$ and $47.5 \%$ in the intervention and control 
groups respectively. The study documented that the use of continuous aspiration of subglottic secretions was useful to decrease the incidence of VAP in high risk populations. The limitation of the study was that it was conducted on a postoperative heart surgery sample in the ICU and findings may not be generalized to other ICU patients.

Kollef et al. (2008) examined whether a silver coated endotracheal tube would reduce the incidence of microbiologically confirmed VAP. In a prospective randomized, single, blinded, controlled trial in 54 centers, patients $(n=2003)$ expected to require mechanical ventilation were randomized to the intervention group (i.e., receive silvercoated endotracheal tube) and control group (uncoated endotracheal tube). The tubes used for intubation were high-volume, low pressure endotracheal tubes that were similar except for the silver coating on the experimental tube. The outcome was measured using incidence of VAP based on quantitative bronchoalveolar lavage fluid culture in intubated patients for 24 hours or longer, time to VAP onset, length of intubation, duration of ICU stay, length of hospital stay, mortality, and adverse events. The findings showed that in patients intubated for 24 hours or more, the incidence of VAP was $4.8 \%$ in the intervention group as compared to $7.5 \%$ in the control group. The silver coated endotracheal tube was associated with delayed onset of VAP. There were no statistically significant differences in duration of intubation, ICU stay, length of hospital stay, mortality, and adverse events. The study identified the successful decrease and delayed onset of VAP with the use of silver coated endotracheal tubes (Kollef et al., 2008) supporting the notion of biofilm proliferation as a causative agent for VAP. 
Grap, Munro, Elswick, Sessler, and Ward (2004) studied the effect of an early post-intubation (i.e., less than 24 hours) one time oral application of chlorhexidine gluconate on oral microbial flora and VAP. The pilot study was conducted in three settings: Emergency Department, Surgical Trauma ICU, and Neuroscience ICU at Virginia Commonwealth University Medical Center. Patients $(n=34)$ who required endotracheal intubation and mechanical ventilation were randomly assigned to chlorhexidine gluconate by spray or swab (treatment group) or to a control group. Oral cultures were done before administration of the intervention, 12 hours after study admission, and every 24 hours up to and including 72 hours after admission to study. The Clinical Pulmonary Infection Score was documented at study admission and at 48 and 72 hours after intubation. The findings showed no statistically significant differences between the three groups. Study trends, on the other hand, showed reductions in oral culture scores only in the treatment group suggesting that the use of chlorhexidine gluconate swabs in the early period post intubation may delay the development of VAP (Grap et al., 2004).

Munro and colleagues (2009) examined the effects of tooth brushing, topical oral chlorhexidine, and oral care using both on the development of VAP in critically ill patients in the ICU with on mechanical ventilation for 24 hours. The randomized control trial assigned patients $(n=547)$ to 1 of 4 treatment groups: $0.12 \%$ solution of chlorhexidine gluconate $5 \mathrm{~mL}$ oral swab twice daily (10 am and $10 \mathrm{pm})$, tooth brushing three times daily ( $9 \mathrm{am}, 2 \mathrm{pm}$, and $8 \mathrm{pm}$ ), combination care of tooth brushing three times daily and chlorhexidine gluconate every 12 hours, or control (i.e., usual care). The development of VAP was measured using the Clinical Pulmonary Infection Score (CPIS) 
where CPIS less than six indicates the absence of pneumonia. The CPIS was assessed on admission to the study (day 1 ) and study days 3,5 , and 7 . By day 3 , pneumonia (i.e., CPIS score more than 6) was found in $24 \%$ of patients in the chlorhexidine gluconate group who had a CPIS score less than 6 on admission to the study. The findings did not document significant effects of chlorhexidine gluconate or tooth brushing. Nevertheless, chlorhexidine gluconate significantly reduced the incidence of pneumonia on day 3 in patients who had CPIS scores less than 6 on day 1 of the study (Munro et al., 2009).

In a study to describe the frequency of pepsin-positive tracheal secretions (a proxy for the aspiration of gastric contents), outcomes associated with aspiration (including a positive CPIS and use of hospital resources), and risk factors associated with aspiration and pneumonia in a population of critically ill tube-fed patients was conducted at five intensive care units in a university-affiliated medical center with level I trauma status. The 2-year study followed 360 adult patients who were on mechanical ventilation and on enteral feedings for 4 days. Approximately $31 \%$ of the sample had a positive pepsin test and at least one aspiration event in $88.9 \%$ of the sample. Monitoring the incidence of pneumonia over the 4 days, findings revealed an increase from $24 \%$ on day 1 to $48 \%$ on day 4. Patients with pneumonia on day 4 had a significantly higher percentage of pepsinpositive tracheal secretions than did those without pneumonia $(42.2 \%$ vs. $21.1 \%$, respectively). Subsequently, the length of stay and need for mechanical ventilation were significantly greater for patients with pneumonia. These findings suggest that aspiration of gastric contents in mechanically ventilated patients with enteral feeding is a major risk factor for VAP. This led to the recommendations and studies that supported nursing interventions to prevent VAP in this population such as head of the bed elevation more 
than 30 degrees (Kieninger \& Lipsett, 2009; Metheny et al., 2006) and the use of protonpump inhibitors (Dodek et al., 2004) to decrease gastric acid reflux and peptic ulcer prophylaxis.

Regarding the use of antimicrobials, de-escalation of treatment is accomplished by changing antibiotics to ones with a narrower spectrum once culture results are finalized. Recommendations for duration of antibiotic therapy have been argued over time and some discrepancies in treatment criteria are still being argued. In a groundbreaking study published by Chastre et al. (2003) patients with VAP were randomized to 8 versus 15 days of antibiotic therapy. Results of the randomized control double blind clinical trial on 401 patients in 51 ICUs showed that death rates were similar between the two groups (18.8\% vs. $17.2 \%)$ and that there was no difference in the overall recurrence rate for VAP (28.9\% vs. $26.0 \%)$. The 15-day group had more antibiotic-free days ( $\mathrm{M}=$ 13.1, $\mathrm{SD}=7.4$ vs. $\mathrm{M}=8.7, \mathrm{SD}=5.2$ days). There was no difference in the number of mechanical ventilation-free days, the number of organ failure-free days, the length of ICU stay, and mortality rates on day 60 for the 2 groups. Although patients with VAP caused by gram-negative bacilli, did not have more adverse outcomes when antimicrobial therapy lasted only 8 days, they did have a higher pulmonary infection-recurrence rate than with those receiving 15 days of treatment ( $40.6 \%$ vs. $25.4 \%$ ). In patients who developed recurrent infections, multi-drug resistant pathogens emerged less frequently in those who had received 8 days of antibiotics ( $42.1 \%$ vs. $62.0 \%$ of pulmonary recurrences). These results suggest that many patients can be safely and effectively treated with shorter course of antibiotics. The implications for establishing adequate treatment courses resulting from this study are of great medical and financial importance. 
Decreasing length of stay, reducing mortality, and reducing overall costs of hospitalization and resource use are the most significant outcomes of implementing such recommendations.

Similarly, Eachempati and colleagues (2009) conducted a study on surgical intensive care unit patients with VAP to investigate whether de-escalation of antibiotics may decrease cost and decrease the development of resistant pathogens, or increase the rate of resistant pneumonia. The findings $(\mathrm{n}=135$ patients $)$ indicated that de-escalation therapy does not lead to recurrent pneumonia or increased mortality in critically ill patients with VAP. In practice, de-escalation therapy may reduce the incidence of bacterial resistance to currently used antibiotic regimens. Using less potent or broad spectrum antibiotics will decrease resistance and will consequently decrease cost. Accordingly, the use of appropriate initial therapy also known as empirical therapy (instituted before confirmative laboratory test) is important for patient outcomes.

All of these preventive measures are compiled and simplified in a VAP bundle that is currently the standard of care in prevention of healthcare-associated pneumonias, specifically VAP. The bundle is comprised of four elements of care supported by solid level I trials. These four elements are: elevation of the head-of-the-bed to between 30 and 45 degrees, daily sedative interruption or "sedation vacation", and daily assessment of readiness to extubate, peptic ulcer disease prophylaxis, and deep venous thrombosis prophylaxis (unless contraindicated) (Institute for Healthcare Improvement [IHI], 2010). Other interventions like oral care and subglottic suctioning of secretions are also included in the bundle. 
Mortality rates for VAP range from 10\%-70\%, increasing the likelihood of death 3-to-4 fold and resulting in extended hospitalizations, excess use of antimicrobials, and increased direct medical costs up to $\$ 30,000$ per case (Coffin et al., 2008; Sole et al., 2002). At a rate of 3 to 10 cases per 1,000 hospital admissions and an overall risk estimated at 3\% per day for the first five days of mechanical ventilation, VAP may increase hospital stay by more than a week resulting in up to $\$ 40,000$ in additional cost and a three-fold increase in mortality (Kieninger \& Lipsett, 2009; Koenig \& Truwit, 2006). Pyenson et al. (2007) reported that the average length of stay increased from $40 \%$ to $47 \%$ when a hospital acquired pneumonia was diagnosed, increasing the inpatient hospital charges by over $39 \%$ on average. The average attributable patient cost of VAP ranges from $\$ 11,897$ to $\$ 25,072$ dollars (Scott, 2009).

Warren and colleagues (2003) studied hospital costs for patients acquiring VAP at medical and surgical intensive care units with patients requiring mechanical ventilation for more than 24 hours. The study results indicated that patients with VAP had higher unadjusted length of stay, hospital length of stay, mortality rate, and hospital costs (127 out of 819 acquired VAP). The hospital costs were significantly higher than uninfected patients $(\$ 70,568$ vs. $\$ 21,620, p<.001)$. Adjusting for severity of illness, multiple regressions revealed that the attributable cost of VAP was $\$ 11,897(\mathrm{p}<.001)$. Similar findings on cost and hospital stay were reported in other studies (Combes et al., 2004; Safdar et al., 2005; Shorr et al., 2006).

Combes et al. (2004) analyzed the impact of methicillin resistance on a large series of patients with Staphylococcus aureus VAP who responded to the initial antibiotic therapy. The charts of patients who were mechanically ventilated 48 hours prior to onset 
of VAP and who exhibited symptoms that were clinically suspicious of VAP were reviewed. These patients also had Staphylococcus aureus growth identified in distal pulmonary samples obtained with a fiber-optic bronchoscope and were started on the appropriate antibiotic therapy within 24 hours after the bronchoscopy. The outcomes were monitored in terms of 28-day mortality, number of mechanical ventilator free days from days 1 to 28 , and durations of mechanical ventilation, and ICU stay after VAP onset. The sample included 171 patients with Staphylococcus aureus VAP with methicillin-resistant Staphylococcus aureus (MRSA) being identified in 74 (43\%) episodes. The findings of the study failed to identify methicillin resistance as a significant indicator for 28-day mortality. Also, there was no significant difference in infection recurrence or superinfections between patients infected by MRSA and patients infected with other organisms. Despite being a retrospective chart review, this study indicates that the use of the appropriate empirical antibiotic therapy for high risk patients early in the course of their hospitalization may lead to improved outcomes. Therefore, costs related to prolonged hospital stay and additional treatment and services would be prevented (Combes et al., 2004).

In a similar study, Shorr and colleagues (2006) conducted retrospective chart reviews to verify the impact of methicillin-resistant Staphylococcus aureus (MRSA) on length of stay in the ICU for patients with VAP who were given appropriate antibiotic therapy for their infection in an attempt to control for the effect of initially inappropriate antibiotic treatment on outcomes. The patients were examined for ICU length of stay, ICU-free days, mechanical ventilation before and after onset of VAP, and duration of mechanical ventilation before and after onset of VAP. The main predictors of the 
prolonged stay were duration of mechanical ventilation before VAP, duration of mechanical ventilation after diagnosis of VAP, and reason for mechanical ventilation. Approximately $33 \%$ of the 107 patients studied were infected with MRSA and had a significantly longer ICU length of stay ( 33 days vs. 22 days; $p=.047)$. The increase in hospital stay in patients with MRSA would lead to additional hospitalization costs even for patients who were started on the appropriate empirical antibiotic therapy earlier (Shorr et al., 2006). These findings contradict the findings of Combes and colleagues (2004) discussed earlier, who found that despite the use of the early appropriate empirical antibiotic therapy, the length of stay, and associated costs were not reduced. These discrepancies continue to generate controversy and debate. Research in this field is needed using for example heterogeneous, multicenter samples of ICU patients controlling for confounding variables such as comorbidities, different surgical interventions, and nursing care protocols.

The incidence of VAP and its impact on the mortality rate, length of stay, and costs was analyzed in a systemic review by Safdar and colleagues (2005). The authors reviewed randomized control trials to study the incidence of VAP and matched cohort studies for the length of stay and cost. The data extracted from the studies compared ICU and hospital mortality due to VAP, prolonged length of stay, and additional days of mechanical ventilation. The findings showed that $10-20 \%$ of patients mechanically ventilated more than 48 hours developed VAP. These patients who developed VAP were twice as likely to die compared with similar patients without VAP, had significantly longer ICU length of stay, and incurred an additional cost of at least $\$ 10,019$ (Safdar et al., 2005). 
Ventilator Weaning. Ventilator weaning is the gradual and progressive withdrawal of the mechanical ventilation support. The weaning process includes the use of different ventilator modes, the allowance of spontaneous ventilation, and rest periods. The discontinuation of mechanical ventilation is important to be achieved once the patient is able to protect their airway while sustaining adequate minute ventilation and physiological stability. Early weaning has been associated with decreased incidence of VAP (Bigatello et al., 2007; Girard et al., 2008; Rello et al., 2002). Because of the potential discomfort provoked by an endotracheal tube and the risk of accidental selfextubation, most mechanically ventilated patients receive sedatives to alleviate the discomfort and prevent self-injury. Unfortunately these medications depress the level of consciousness as well as the respiratory rate. Girard et al. (2008) assessed a protocol that paired spontaneous awakening trials (i.e., daily interruption of sedatives) with spontaneous breathing trials. In four tertiary care hospitals, mechanically ventilated patients in the ICU $(n=336)$ were randomly assigned to management with daily spontaneous awakening trials followed by spontaneous breathing trials or to the usual care that included sedation with spontaneous breathing trials. The goal was to achieve breathing without assistance. Patients in the control group were assessed every morning for spontaneous breathing safety screen that included adequate oxygenation, spontaneous inspiratory effort in 5-minute period, absence of agitation, and others. Patients who passed had the ventilator support removed and were allowed to breathe through a T-tube or a ventilator circuit with positive airway pressure of $5 \mathrm{~cm} \mathrm{H} \mathrm{H}_{2} \mathrm{O}$. Patients in the intervention group were also assessed every morning with a spontaneous awakening safety screen that included the absence of sedation for active seizure or alcohol 
withdrawal, receiving escalating sedative dose due to ongoing agitation, receiving neuromuscular blockers, and others. Patients who passed had all their sedatives and analgesics stopped with the exception of those used for pain. Patients were monitored for up to 4 hours and considered to pass if they opened their eyes to verbal stimuli or tolerated sedation interruption for 4 hours or more without exhibiting signs of failure (i.e., anxiety, agitation, pain, respiratory rate more than 35 per minute for 5 minutes, acute dysrhythmia, hypoxia, or respiratory distress). The endpoint was defined by the number of days patients were breathing without assistance (ventilator-free days) during the 28study period. The findings showed that patients in the intervention group had significantly more ventilator free days during the 28 -study period than those in the control group (14.7 days vs. 11.6 days). More patients in the intervention group self extubated than those in the control group. This study documented that wake up and breath protocol that includes daily spontaneous awakening trials with daily spontaneous breathing trials has better outcomes for mechanically ventilated patients in the ICU (Girard et al., 2008).

In a longitudinal study of outcomes of 210 critically ill patients undergoing prolonged mechanical ventilation at an acute care respiratory unit, researchers found that a substantial portion of these patients were unable to wean from ventilator use, resulting in increased mortality. The average time to extubation was 14 days with an increased length of stay of 58 days for those who were unable to be weaned. The majority of the sample was ventilated through a tracheostomy $(79 \%)$, followed by orotracheal tube $(19 \%)$, and facemask (3\%). Those who were unable to be weaned off the ventilator had increased mortality and were more likely to die within a year (odds ratio [OR], 6.5). At 6 
months following discharge, $69 \%$ of the sample was off mechanical ventilation. Those who were unable to be weaned had preexistent respiratory comorbidities $(\mathrm{OR}, 0.50)$ and readmissions to the respiratory unit (OR, 3.84). Patients who had successful weaning had neurologic comorbidities (OR, 3.84) and unsuccessful extubation before admission to the respiratory unit (OR, 2.67). Approximately, three quarters $(75 \%)$ of the deaths were due to discontinuation of life support after prolonged intubation (Bigatello et al., 2007). Consequently, early extubation is important for patient mortality and outcomes.

Ventilator Days. A ventilator day is a metric used for reporting and benchmarking the number of days a patient is connected to a ventilator. This measure is essential in evaluating the effectiveness of interventions aimed to reduce the number of days patients remain intubated or ventilator dependent. In addition, it could be used as an outcome measure to reduce VAP, ICU LOS and cost.

In a large retrospective study on 9,080 patients admitted to the ICU and receiving mechanical ventilation for more than 24 hours, Rello and colleagues (2002) examined the risk factors, in-hospital mortality, resource utilization, and hospital charges. The patients with VAP were matched with a control group of mechanically ventilated patients without VAP. The findings revealed that patients with VAP had significantly longer mechanical ventilation $(M=14.3, S D=15.5$ vs. $M=4.7, S D=7.0$, days $), I C U$ stay $(M=11.7, S D=$ 11.0 vs. $\mathrm{M}=5.6, \mathrm{SD}=6.1$, days $)$, and hospital stay $(\mathrm{M}=25.5, \mathrm{SD}=22.8$ vs. $\mathrm{M}=14.0$, $\mathrm{SD}=14.6$, days). This was consequently associated with increased hospital charges of more than $\$ 40,000$ per patient $(M=104,983, S D=91,080$ vs. $M=63,689, S D=75,030)$. The findings of this study support the importance of preventing VAP in improving patient outcomes and decreasing cost (Rello et al., 2002). 


\section{Catheter Associated Urinary Tract Infections (CAUTI). Urinary tract}

infections account for close to $40 \%$ of all healthcare-associated infections (Lo et al., 2008). Eighty percent of all urinary tract infections, the most common healthcare associated infection, are catheter-associated urinary tract infection (CAUTI) (Lo et al., 2008). Urinary catheters represent a daily risk of infection of $3 \%$ to $5 \%$ when an indwelling catheter remains in situ (Lo et al., 2008) and are associated with increased death rates, prolonged hospital stays, and increased costs adding as much as $\$ 1,000$ to the direct cost of acute-care hospitalizations (Maki \& Tambyah, 2001). Urinary catheters are frequently used among critically ill patients in ICUs for accurate measurement of urinary output or for patients undergoing genitourinary surgeries requiring an invasive drainage. Reducing the length of time of indwelling catheterization, one of the most important modifiable risk factors for CAUTI, will reduce the mortality, length of stay, and cost associated with this condition (Lo et al., 2008; Maki \& Tambyah, 2001). Additional risk factors include female sex, older age, and not maintaining a closed drainage system (Lo et al.). Also pregnant women, diabetics, malnourished, and azotemic (i.e., creatinine > 2.0mg/dL) patients are at higher risk of developing CAUTI (Maki \& Tambyah, 2001).

To understand the infectious process associated with CAUTI, it is important to evaluate the pathogenesis of the entity. Colonization of urinary catheters is almost inevitable and may reach concentrations greater than $10^{5} \mathrm{cfu} / \mathrm{mL}$ within 72 hours (Maki \& Tambyah, 2001; Stark \& Maki, 1984). The most likely portal of entry of microorganisms is extraluminal, making females more susceptible to these infections given their shorter urethral tract (Maki \& Tambyah, 2001). The resulting biofilm in the catheter serves as a host to gram-negative and gram-positive organisms, as well as to 
yeast, creating resistance to antimicrobial agents and protecting the causative factors from the body's own defense system (Stickler \& Morgan, 2008).

Most pathogens of CAUTI arise from the patient's perineal and colonic flora or from the hands of healthcare providers (Maki \& Tambyah, 2001). Pathogens may travel also intraluminal from the collecting bag or the connecting tubing. The drainage bag is potentially a large reservoir for pathogenic organisms that can be transmitted to other patients (Lo et al., 2008). The most frequent pathogens associated with CAUTI are Escherichia coli (21.4\%) and Candida spp. (21.0\%), Enterococcus spp. (14.9\%), Pseudomonas aeruginosa (10.0\%), Klebsiella pneumoniae (7.7\%), and Enterobacter spp. (4.1\%). A smaller proportion accounted for other gram-negative bacteria and Staphylococcus spp. (Gould et al., 2009).

The most common clinical presentation of CAUTI is fever (temperature greater than $38^{\circ} \mathrm{C}$ ) and positive urine cultures or organisms seen on Gram's stain of unspun urine without any other localizing findings (Horan et al., 2008; Lo et al., 2008). A microbiological diagnosis usually requires the growth of at least two urine cultures of at least $105 \mathrm{cfu} / \mathrm{mL}$ of a single organism in urine collected aseptically with an e from the sampling port of the urinary catheter (Horan et al., 2008; Lo et al., 2008; Maki \& Tambyah, 2001; Stark \& Maki, 1984). The diagnosis is complemented with findings of leukocytosis in urine and sometimes in serum. Laupland and colleagues (2005) described the occurrence, microbiology, and risk factors for acquiring CAUTI in the ICU in a surveillance cohort study. The study was conducted over a 3-year period during which 4,465 patients were admitted 4,915 times to an ICU for 48 hours or more. CAUTI incidence was observed in $7 \%$ of patients resulting in an overall incidence of CAUTI of 
9.6 per 1,000 ICU days. The development of CAUTI in the ICU was more common in women and medical patients (compared to surgical patients). The findings of this study highlighted the commonality of developing CAUTI in ICU in critically ill patients.

Leone et al. (2001) conducted a prospective nonrandomized controlled trial to determine whether the rate of acquisition of bacteriuria differed between the use of complex closed drainage system (pre-attached catheter, antireflux valve, drip chamber, and povidone iodine releasing cartilage) and two-chamber open drainage system in ICU patients. The study was conducted during two consecutive periods (6 months each) in which the two-chamber open drainage system and complex closed drainage system were used successively. During 12 months, 224 patients were enrolled in the study (111 in the complex closed drainage system and 113 in the two-chamber open drainage system). The use of antimicrobials was similar between the two groups. The mean duration of urinary catheterization was 8 days ( $\mathrm{SD}=7$ days) in the two-chamber open drainage system and 9 days ( $\mathrm{SD}=7$ days) in the complex closed drainage system. Findings indicated that catheter related bacteriuria occurred in 27 of 224 patients (12\%) (11.5\% in the twochamber open drainage system group and $13.5 \%$ in the complex closed drainage system group). There was no statistically significant difference in the incidence between the two groups. Bacteriuria was diagnosed on days $14(\mathrm{SD}=8)$ and $13(\mathrm{SD}=9)$ of catheterization for the two-chamber open drainage system and complex closed drainage system, respectively. This was one of the first studies to compare the effectiveness of the two drainage systems in ICU patients. A major limitation of this study was the absence of randomization despite the authors reporting no changes in the ICU during the two consecutive periods. 
Treatment of CAUTI is aimed more to prevention. Urosepsis usually requires aggressive antibiotic therapy, supportive care, and elimination of the causative agent, in which case early discontinuation of the urinary catheter proves to be the best preventative measure (Cravens \& Zweig, 2000). In a study conducted by Brosnahan, Jull \& Tracy (2004) to determine what type of catheter ideally should be used to prevent CAUTI, the results suggested that the use of silver alloy indwelling catheters for short-term catheterization reduces the risk of CAUTI (RR: $0.36,95 \%$ CI: 0.24 to 0.52 [for asymptomatic bacteriuria]) and RR: $0.60,95 \% \mathrm{CI}$ : 0.50 to 0.73 [for symptomatic bacteriuria]).

Elpern et al. (2009) implemented and evaluated the efficacy of an intervention to reduce CAUTI in a medical ICU by decreasing the use of urinary catheters. Unit clinicians developed guidelines for continuing indwelling catheterization. All consecutive patients admitted to the ICU and had an indwelling catheter during their stay were enrolled in the study. Daily evaluations of indwelling catheters using criteria for appropriate use of catheters were conducted by nurse investigators (APNs).

Recommendations were made to discontinue catheters in patients who did not meet the identified criteria. Data were collected on duration of catheterization, appropriateness of urinary catheterization, and reasons for inappropriate catheter use. Surveillance for CAUTI was also collected. The days of catheter use and rates of CAUTI were compared with those of the preceding 11 months. During the 6 month-intervention period, 337 patients with indwelling catheters for a total of 1432 catheter days were enrolled in the study. Using the guidelines, the catheter duration was reduced significantly from a mean of $311.7(\mathrm{SD}=56.4)$ to $238.6(\mathrm{SD}=30.2)$ days per month. During the intervention 
period, the rate of CAUTI per 1,000 days was reduced from $4.7(\mathrm{SD}=2.5)$ per month to zero. An intervention to evaluate the appropriateness of indwelling urinary catheterization reduces CAUTI (Elpern et al., 2009).

Many institutions have adopted the recommendations of several agencies and organizations dedicated to promote prevention and elimination of HAIs. These recommendations have been made operational and instituted as care bundles for HAIs including CAUTI. Contrary to the bundles for VAP and CLABSI, for CAUTI there is little consensus on the adoption of specific measures, and more criteria are needed for CAUTI prevention. The most extensive and inclusive list was published by the Association for Professionals in Infection Control and Epidemiology (APIC) in 2008. This list includes: the use of indwelling bladder catheters only when medically necessary, use aseptic insertion technique with appropriate hand hygiene and gloves, maintain a sterile closed drainage system, maintain good hygiene at the catheter-urethral interface, remove catheters when no longer needed, document indication for urinary catheter on each day of use, and the use of reminder systems to target opportunities to remove catheters.

Nurse-led interventions are critically important, not only in implementing the evidence based practice bundles, but generating data that evaluates these interventions. Fakih et al. (2008) reported the effect of nurse-led multidisciplinary rounds on reducing the unnecessary use of urinary catheterization in hospitalized patients. The study tested the effect of nurse-led multidisciplinary rounds in a major teaching hospital. Of almost 5,000 patient-days, a urinary catheter was present in 885 . The study findings revealed that the multidisciplinary rounds led to a decrease in the unnecessary use of catheters but 
it was not sustained after the post intervention phase (Fakih et al., 2008). Despite the success of the intervention, sustainability of implementing evidence-based interventions is essential to maintain outcomes.

Huang and colleagues (2004) evaluated the efficacy of nurse- generated daily reminders to physicians to discontinue unnecessary urinary catheters in adult ICU patients. The study findings indicated that catheterization was significantly reduced during the intervention phase $(\mathrm{M}=7.0, \mathrm{SD}=1.1$ days to $\mathrm{M}=4.6, \mathrm{SD}=0.7$ days, $\mathrm{p}<$ .001). In addition, the CAUTI rate was significantly reduced $(\mathrm{M}=11.5, \mathrm{SD}=3.1$ to $\mathrm{M}=$ $8.3, \mathrm{SD}=2.5$ patients with CAUTI per 1000 catheter days, $\mathrm{p}=.009$ ). This study builds on the importance of implementing evidence-based interventions of early discontinuation of urinary catheters and emphasizes the significance ensuring compliance through interventions such as daily reminders (Huang et al., 2004).

Rosenthal, Guzman, and Safdar (2004) evaluated the effect of education and performance feedback regarding compliance with catheter care and hand washing on rates of CAUTI in the ICU. The study was conducted at a 180-bed private hospital with two 10-bed ICUs. Sterile closed urinary drainage systems were used for all catheterizations during the study. Patients with indwelling catheters for more 24 hours in the ICUs were enrolled in the study. Data were collected on age, gender, average severity of illness score, duration of catheterization, antibiotic use, use of other invasive devices, and other sites of infection during the catheterization duration. The intervention included education and performance feedback. Education was conducted regarding hand hygiene as published by the $\mathrm{CDC}$ and Healthcare Infection Control Practices Advisory Committee (Gould et al., 2009). Performance feedback was initiated during the 
intervention period regarding compliance with infection control practices and was provided monthly. During the study, 1,301 patients in the ICUs required indwelling catheters and were enrolled in the study. During the first two months of the study or the pre intervention period baseline data were collected. During the intervention period a total of 5,568 catheter days were documented. Comparison between the baseline and intervention periods showed that compression of the catheter by leg improved significantly from $83 \%$ to $96 \%$. Compliance with hand washing improved significantly from $23.1 \%$ to $65.2 \%$. The CAUTI rate decreased significantly from 21.3 to 12.39 per 1,000 catheter days. Implementing an education and performance feedback intervention regarding catheter care measures and hand washing compliance contributed to decreasing CAUTI rates (Rosenthal et al., 2004).

Despite the available evidence pointing to reducing the use and permanence of indwelling urinary catheters as a measure of prevention of CAUTI, indwelling bladder catheter use continues to prevail. The consequent implementation of other preventive measures when the use of catheters is justified remains debatable. The lack of consensus in guidelines and standards of care for indwelling catheters and CAUTI prevention still prevails. This differs from other HAIs bundles which are recognized as gold standards in care.

Research has shown that there are many different dimensions in the study of HAIs. Healthcare Associated Infections' complex nature may allow studying the phenomena from different angles and perspectives (e. g., epidemiology, medicine, infection control, public health, and nursing). This myriad of commonalities among disciplines creates a rich endeavor for researchers to collaborate in advancing knowledge 
and decreasing the knowledge gap in HAIs. In the past decade studies identified best practice efforts to help curtail the incidence and prevalence of HAIs, resulting in the creation of bundles for practice implementation. However, the challenge remains to continue improving these best practices, developing tools to measure compliance with the implementation of these practices, and continue disseminating the knowledge base on HAIs prevention and interventions.

Urinary catheters represent a daily risk of infection of $3 \%$ to $5 \%$ when an indwelling catheter (i.e. e., Foley catheter) remains inserted (Lo et al., 2008) and are associated with increased death rates, prolonged hospital stays, and increased costs adding up to $\$ 1,000$ to the direct cost of acute-care hospitalizations (Maki \& Tambyah, 2001). Currently, CAUTIs comprise the highest percentage of all HAIs (34\%) reported in the U.S. (Klevens et al., 2007; HHS, 2009). Costs associated with CAUTI range between \$589 and \$758 dollars per infection (Scott, 2009).

In one of the first large studies to prospectively examine the hospital cost of CAUTI, Tambyah, Knasinski, \& Maki (2002) conducted a cost analysis on newly catheterized hospitalized patients subsequently diagnosed with CAUTI $(n=123)$. The study reported that patients diagnosed with CAUTI incurred in an additional \$20,662 in extra cost for laboratory tests and $\$ 35,872$ in extra medication costs resulting in an average $\$ 589$ (SD = 1,265) per CAUTI (in 1998 dollars) (Tambyah et al., 2002).

Pressure Ulcers. Pressure ulcers remain a main concern in the ICU. Critically ill patients in ICUs are at higher risk for developing pressure ulcers. Although pressure ulcer incidence in the ICU can be reduced, interventions have not yet succeeded in avoiding their development. Reports have published incidences of pressure ulcers in the 
ICU to range from one to 56\% (Keller et al., 2002; Jiricka, Ryan, Carvalho, \& Bukvich., 1995; Schoonhoven et al., 2002). The main reasons behind the increased incidence of pressure ulcer in ICU patient are immobilization, sedation, and the use of mechanical ventilation (Defloor et al., 2005). Pressure ulcers increase the risk of mortality and morbidity (Keller et al., 2002), infection, and sepsis (Edwards, 1994). Also, ICU patients generally may suffer from impaired circulation, especially the geriatric population, or the use of vasoactive drugs which increases their risk of developing pressure ulcers (Keller et al., 2002).

Shahin, et al. (2009) assessed the allocation of preventive measures for patients at risk of developing pressure ulcers. The study also examined the evidence of applied preventive measures in the ICU according to the European Pressure Ulcer Advisory Panel (EPUAP) and Agency for Health Care Policy and Research (AHCPR). The crosssectional study was conducted in the ICU with 169 patients recruited from surgical $(n=60)$, interdisciplinary $(n=59)$, and medical $(n=50)$ adult ICU wards from 18 hospitals. Data were collected on patient demographics, pressure ulcer occurrence, grades, body sites of pressure ulcers, duration, origin, types of dressing, and preventive measures. The EPUAP grading system and Braden scale for risk assessment were used in the study. The findings of this study showed that $83 \%$ of patients were at risk for developing pressure ulcers using the Braden scale. The prevalence of pressure ulcers was reported to be $27.2 \%$ where the highest was among surgical patients (39\%) and the lowest was among the interdisciplinary patients (18\%). Preventive measures used were pressure reducing devices including special mattresses (36.5\%), special beds (2.5\%), and special cushions (17.6\%). Nursing interventions that were implemented included repositioning (41.5\%), 
mobility (56.6\%), skin inspection (81.8\%), massage with moisturizer cream (80.5\%), avoidance of nutritional and fluid deficit (68.6\%), patient education (40.3\%), family education (20.8\%), avoidance of sheer and friction (32\%), and massage (8.8\%). Mattresses (i.e., alternating pressure air, low air loss, and foam) were the most used preventive measures in at risk patients. Patients who were identified to be at risk for developing pressure ulcers were found to have more than one nursing intervention implemented. The findings documented that there was a significant difference in the allocation of preventive measures between the at-risk and not at-risk patient groups for pressure ulcer. The pressure ulcer preventive measures used were in congruence with those identified by the EPUAP and AHCPR guidelines with the exception of massage that was used with $8.8 \%$ of patients. The guidelines have suggested avoiding the use of massage as a preventive measure for pressure ulcers. A major limitation of this study was that the sample did not include unconscious patients and the sample size was rather small (Shahin et al., 2009).

Compton and colleagues (2008) examined the routinely documented ICU-specific objective and subjective parameters to predict the development of pressure ulcers in the ICU. The authors evaluated the objective parameters and subjective nursing assessment of pressure ulcer risk factors for ICU patients, and compared them with the performance of the Waterlow scale. Patients who were free from pressure ulcers and admitted to the medical ICU were included in the study. Patients with LOS in the ICU less than 72 hours were excluded from the study. Pressure ulcer grading was performed using the EPUAP. Pressure ulcer risk factors included parameters that were identified and documented within the first 24 hours of admission to the ICU. Data were collected from the 
subjective nursing assessment including the Waterlow scale completed by the admitting nurse, in addition to objective clinical, monitoring, and laboratory parameters documented within the first 24 hours. The study analysis was conducted using data from 698 patients admitted to the ICU with a median stay of six days and a mortality of $22.1 \%$. Within a median time of seven days, 121 patients developed pressure ulcers in the ICU. The ICU LOS was longer (median 24 vs. 5) for patients developing pressure ulcers as compared to those who do not develop pressure ulcers. The ICU mortality was higher in patients developing pressure ulcers (44.6\% vs. $17.3 \%)$. Organ dysfunction, circulatory impairment, and sepsis were significantly associated with the occurrence of pressure ulcers. Logistic multiple regression showed that subjective nursing skin assessment was a significant predictor of the risk of developing pressure ulcers. The findings of this study emphasize the importance of nursing skin assessment in the prediction of pressure ulcers in the ICU (Compton et al., 2008).

Lahmann, Kottner, Dassen, and Tannen (2012) evaluated the effect of being treated in the ICU in comparison with other hospital units regarding pressure ulcer occurrence controlling for risk factors. The SRISAG (surface, repositioning, immobility, shear force, age, and gender) model was used to control for the risk factors. Pressure ulcers were assessed based on the EPUAP classification. Patients $(n=32,400[30,163$ from mixed wards and 2,237 from ICUs]) were recruited from 256 hospitals. Pressure ulcers $(n=4,812)$ were identified in 2,766 patients on hospital wards and 521 patients in ICUs where $38.4 \%$ and $61.4 \%$ were developed on the identified units, respectively. The prevalence of ward and ICU acquired pressure ulcers were found to be $3.9 \%$ and $14.9 \%$ respectively. Logistic regression to assess the contribution of age, sex, appliance of 
turning intervals, use of special surface, shear and friction, impaired mobility, and organizational factors (i.e., ward vs. ICU) to the development of pressure ulcers on the units. The model was significant and the highest odds ratio was for patients at risk for friction and shear. Patients in the ICU had a higher probability for developing pressure ulcers (1.5 times higher). The findings of this study suggested that patient specific factors and implementation of preventive interventions contribute to the higher rate of pressure ulcers incidence in the ICUs. Therefore, increasing the awareness and providing adequate interventions in the ICU may help in decreasing the occurrence of pressure ulcers in the ICU population (Lahman, et al., 2011).

The incidence, risk factors, and screening of pressure ulcers in ICU patients were examined. The study was conducted using 142 patients admitted to the ICU. Pressure ulcer screening was completed within the first two hours of admission and after discharge from the ICU using the National Pressure Ulcer Advisory Panel (NPUAP) guidelines. Norton scale was used to assess the pressure ulcer risk. Age, nutritional screening, hospitalization period, mean arterial pressure, pressure ulcer degree, hemoglobin and albumin levels, APACHE-II scores, body mass index, and comorbidities data were collected. The pressure ulcer incidence was $7.8 \%$ which was explained by the authors to be the result of the strict screening of patients within the first two hours of admission to the ICU. Norton score, long hospitalization, hypotension, malnutrition, and hypoalbuminemia, age, high APACHE-II score, and medications were significant in patients developing pressure ulcers in the ICU (Terekeci et al., 2009).

Cox (2011) determined which risk factors (i.e., Braden scale total score on admission, mobility, activity, sensory perception, moisture, friction and shear, nutrition, 
age, blood pressure, ICU LOS, APACHE-II score, vasopressor administration, and comorbid conditions) are the most predictive of pressure ulcers in adult critical care patients. In a retrospective correlational study, 347 patients admitted to the adult medical surgical ICU for more than 24 hours were examined. Pressure ulcer was documented in $18.7 \%$ of patients. Patients developing pressure ulcers were identified as at risk $(28 \%)$, moderate risk (28\%), high risk (35\%), and very high risk $(9 \%)$. The majority of the ulcers was Stage II (35\%) and developed on the sacrum (58\%). The ulcers developed within an average of 133.61 hours $(\mathrm{SD}=120.13)$. Logistic regression showed that mobility, age, ICU LOS, and cardiovascular disease were significant predictors of pressure ulcers. Stage II or greater pressure ulcers were significantly predicted by friction and shear, ICU LOS, norepinephrine administration, and cardiovascular disease (Cox, 2011).

Length of Stay (LOS). Length of stay (LOS), specifically in the ICU setting, can be linked to patient outcome and healthcare cost. In addition, it may represent indirect burden to patients, patient families, and society in general (Gruenberg et al., 2006). In a study to assess the independent effect of ICU LOS on in-hospital and long-term mortality after hospital discharge, Williams and colleagues (2010) examined the clinical and mortality data of 22,298 adult ICU patients. Data were collected from a 22-bed ICU of a tertiary teaching hospital over a five year period. Cox regression with restricted cubic spline function was used to analyze the data. The model aimed to predict the effect of ICU LOS on in-hospital and long-term mortality after adjusting for age, gender, acute physiology score (APS), maximum number of organ failure, era of admission (i.e., 19871990, 1991-1994, 1995-1998, 1999-2000), elective admission, Charlson's comorbidity 
index and diagnosis. Patients staying in the ICU for 10 days or less were the major proportion of the surviving patients in the study (94\%). The median ICU LOS was 17 days. The majority of patients staying more than 10 days in the ICU were septic or trauma victims and had more severe conditions as indicated by the APS and number of organ failures. The findings showed that after adjusting for age, gender, APS, number of organ failure, year of admission, elective admission, Charlson's comorbidity index and diagnosis, increased admission was not related to increased hospital mortality. The majority of the deaths occurred during the first 10 days of ICU stay. There was an association between long term mortality risk and increased LOS (e.g., up to 10 days). The long term mortality risk stabilized after the 10 day ICU LOS period. Age, comorbidities, diagnosis, and APS were found to better predict long term mortality than ICU LOS. This study suggested that ICU LOS was not an independent factor affecting mortality of patients in the hospital. However, ICU LOS contributed to the long term mortality after hospital discharge after adjusting for other risk factors (Williams et al., 2010). A major limitation of the study is that data were collected over 16 years. During this long period there may have been changes in the ICU patient characteristics and management that may have influenced the study findings. Also, the data were collected from one center.

Wise and colleagues (2011) compared mortality and LOS of medical ICU patients cared for by a hospitalist and an intensive-led team. In a prospective observational study, 828 consecutive medical ICU patients admitted to a hospitalist team and 528 patients admitted to an intensivist ICU teaching team were examined. Data about hospital and ICU mortality, hospital and ICU LOS, and patient characteristics and outcomes were 
collected. The mean ICU LOS for the total sample was 4 days $(\mathrm{SD}=5.9)$ and the mean hospital LOS was 9.1 days $(\mathrm{SD}=9.0)$. Approximately $12.4 \%$ of the total sample died and $10 \%$ of those deaths occurred in the ICU. Based on the simplified acute physiology score (SAPS II) patients were categorized into low intermediate, and high disease acuity. The findings indicated that patients identified with low and high acuity and treated by the intensivist led team had lower mortality and LOS. The hospitalist-led team has lower mean SAPS II (37.4 vs. 45.1$)$, less noninvasive procedures (17.9\% vs. $25.8 \%$ ), less mechanical ventilation ( $11 \%$ vs. $51.9 \%)$, and fewer central venous catheters $(29.1 \%$ vs. $50.8 \%)$. There were no differences in adjusted LOS between the two teams. Mechanically ventilated patients with intermediate illness severity were found to have lower hospital (1.6 vs. 17.8) and ICU (7.2 vs. 10.6) LOS (Wise et al., 2011).

Kramer and Zimmerman (2010) developed and validated a model that identified patients at risk for prolonged ICU LOS. The retrospective cohort study reviewed a total of 343,555 charts of admissions to 83 ICUS in 31 U.S. hospitals. The ICU LOS was reviewed and a five day cut point was identified as a time-point after which prolonged LOS was a concern. The five day cut point was chosen because it allowed time to show complications and responses to therapy. This five day cut point represented the $80^{\text {th }}$ percentile of ICU admissions. Patients with more than five days ICU LOS were found to have significantly higher severity of illness, frequency of mechanical ventilation, emergency surgery, and ICU readmissions. These patients had poorer outcomes and accounted for $21 \%$ of ICU admissions and $63 \%$ of total ICU days. Patients with more than five days LOS in the ICU were examined using a multivariate regression model to predict the remaining ICU stay. The variables included in the model were: APS, chronic 
health items (i.e., AIDS, cirrhosis, hepatic failure, immunosuppression, lymphoma, leukemia, myeloma, metastatic tumor), ICU admission diagnosis, ICU admission source, LOS before ICU admission, age, $\mathrm{PaO}_{2}$ on day one, emergency surgery, ventilated day one, inability to assess Glasgow coma on day one, thrombolytic therapy, rescaled Glasgow coma score on day one, day one ICU LOS prediction, readmission to ICU, APS, ventilated on day five, inability to assess Glasgow coma scale on day 5, $\mathrm{PaO}_{2}$ on day five, rescaled Glasgow coma scale on day five, and APS difference between days four and five. Mechanical ventilation and inability to measure Glasgow coma on day five accounted for $48.8 \%$ of the model's explanatory power. The model showed that the variables measured on day five predicted the remaining ICU LOS. Mechanical ventilation $\mathrm{PaO}_{2}$, physiologic components, and sedation on day five accounted for $81.6 \%$ of the variation in the predicted ICU stay (Kramer \& Zimmerman, 2010). The findings of this study suggested the use of patient outcomes of day five to predict ICU stay and identify alternatives to reduce the ICU LOS. The use of APN Intensivists in the ICU may contribute to decrease LOS. APN Intensivists-led interventions such as surveillance of sepsis, and assessment and management of HAIs, pressure ulcer, post-operative glycemic control, and sedation have not been documented and are important to be examined.

Patient Initiated Removal of Devices. Catheter devices (i.e., urinary catheter, central venous catheter, endotracheal tube, arterial catheter, and thoracic drain) are common among critically ill patients in the ICU. In addition to the risk of developing a hospital acquired infection related to these devices, accidental removal of these catheters is another risk factor. The risk of the accidental removal is in the consequences of the removal itself in addition to those related to the catheter reinsertion. Lorente, Huidobro, 
Martin, Jimenez and Mora (2004) analyzed the incidence of accidental removal of devices for all types of catheters in the ICU. The prospective observational study included all consecutive patients $(\mathrm{n}=988)$ admitted to a 24-bed ICU for a period of 18 months. Data were collected on all catheters per 100 catheters and 100 catheter days. The findings did not suggest a difference in the incidences between the central venous catheters (i.e., peripheral, jugular, subclavian, and femoral) and between the arterial access sites (i.e., radial, femoral, pedal, and humeral). When comparing venous and arterial access devices, there was a significant difference with a higher incidence of accidental removals for the central venous catheters $(1,012$ per 100 catheter days versus 2.02 per 100 catheter days). The accidental removal of catheters per 100 days for endotracheal tubes was .79 , nasogastric tubes was 4.48 , urinary catheters was .32 , thoracic drain was .56, abdominal drain was .67, and intraventricular brain drain was .66 (Lorente et al., 2004).

Fraser et al. (2001) determined the frequency and pattern of the accidental removal of medical devices in the ICU. In a prospective observational study at a two 10bed multidisciplinary ICU, adult patients admitted for longer than 24 hours were recruited for the study. A total of 36 patients and 199 patient days were studied. Medical records of these patients were examined for the occurrences of patient initiated removal of devices. The responses of healthcare providers to these removals were also documented. Costs related to these removals were also examined using hospital databases and Medicare physician reimbursement schedules. The findings of the study showed that $28 \%$ of patients removed 42 devices. The majority of these devices $(88 \%)$ were gastrointestinal tubes and vascular catheters. Before most of the removals $(74 \%)$, 
significant patient agitation was documented in the chart within two hours of the accident. The annual cost associated with these removals was estimated to be more than $\$ 250,000$ or $\$ 7,606$ per device, or $\$ 181$ per event (Fraser et al., 2001).

Carrion and colleagues (2000) examined the rates of accidental removal of endotracheal tubes, nasogastric tubes, central venous catheters, and arterial catheters in a prospective, observational, and intervention study. The authors also assessed the efficacy of the corrective measures taken to reduce the accidental removal of these devices. Patients admitted to the adult ICU and having one of the aforementioned devices for more than 24 hours were included in the study. Data collected on the devices included date of placement, position, date of removal, and reason for removal. Data were collected for three consecutive 6-month periods. At the end of the first two time periods, findings were shared with the physicians and nurses. Instructions and specific measured to reduce the accidental removal of these devices were implemented. The findings showed that 289 endotracheal catheters were placed and $13.1 \%, 17.1 \%$, and $11.4 \%$ were accidentally removed during the first, second, and third time periods respectively. A total of 368 nasogastric tubes were initiated and accidental removal significantly decreased among $41 \%, 32.4 \%$, and $25.8 \%$ of patients during the first, second, and third time periods respectively. Similarly, there was a significant decrease in the incidences of central venous and arterial catheter removals (Carrion et al., 2000).

Mion and colleagues (2007), in a prospective prevalence study, determined the prevalence of device removal, described the patent contexts, and examined the unit level adjusted factors associated with the patient initiated removal of devices. Data were collected from 49 adult ICUs from 39 hospitals. The data included a total of 49,482 
patient days in addition to information about unit census, ventilator days, restraint days, and days accounted for by men and by elderly patients. The findings indicated that 1,623 devices were removed accidentally by patients on 1,097 occasions. The overall rate of patient initiated removal of devices was 22.1 episodes per 1,000 patient days. The surgical ICU had a lower incidence of removals (16.1 episodes) compared to the general (23.6 episodes) and medical (23.4 episodes) ICUs. More than half of the episodes were accounted for by men (57\%) who were restrained at the time (44\%) and not receiving any sedation (30\%) in the 24 hours prior to the accident. Self extubation was inversely associated with ventilator days. Patient harm was documented in $23 \%$ of the episodes and reinsertion rates depended on the device. Reinsertion of devices ranged from around $23.5 \%$ of surgical drains to $88.9 \%$ of monitor leads (Mion et al., 2007).

Sedation Management. Sedation is frequently used with ICU patients who are on mechanical ventilation. Sedation is required to decrease the discomfort of mechanical ventilation, suctioning, and other procedures. Sedation minimizes anxiety and provides comfort and rest to mechanically ventilated patients. Also, sedation helps reduce the metabolic response to surgery. The majority of the drugs used for sedation include benzodiazepines, propofol, and haloperidol. These drugs have been used following strict guidelines to minimize their side effects (Kress et al., 2000). Schweickert and colleagues (2004) examined the effect of daily interruption of sedative infusions. This was associated with a decrease in complications (i.e., VAP, upper gastrointestinal hemorrhage, bacteremia, barotrauma, venous thromboembolitic disease, cholestasis, and sinusitis). A total 126 patients using mechanical ventilation and continuous sedative infusion in a medical ICU were included in the retrospective blinded chart review study. 
Data were compared between a group of patients who received daily interruption of sedative infusions $(n=66)$ and those receiving sedation as prescribed by the medical ICU team $(n=60)$. The acute APACHE II scores were similar in both groups. Thrombosis prophylaxis was administered to the majority of patients $(90.2 \%$ of patients in daily interrupted sedation and $92.5 \%$ in control group). Gastric stress ulcer prophylaxis was also administered to the majority of patients $(90.5 \%$ of patients in daily interrupted sedation and $96.3 \%$ in control group). The findings of the study showed that the daily interrupted sedation group had a total of 13 complications in 12 patients. Patients in the control group had a total of 26 complications in 19 patients (Schweickert et al., 2004).

Kress et al. (2000) conducted a similar study examining daily interruption of sedation in a randomized controlled study. The study was conducted in the medical ICU with 128 mechanically ventilated patients. The intervention group received daily interruption of sedation. The control group received the regular care or sedation management as ordered by the medical team. The findings of the study showed that patients receiving the interrupted sedation protocol had a significantly shorter duration of mechanical ventilation (4.9 days vs. 7.3 days). The intervention group had a shorter ICU LOS (6.4 days vs. 9.9 days). These findings suggested positive outcomes of using sedation interruption protocols on critically ill and mechanically ventilated patients in the ICU (Kress et al., 2000).

Mascia and colleagues (2000) conducted a prospective two phase study examining the pre- and post-effect of a sedation management protocol. The authors identified the rational for cost effective use of the drug. Data were collected with 158 mechanically ventilated patients from a medical and surgical ICU. The findings 
suggested that the guideline group had a shorter mechanical ventilation duration (1678 hours vs. 317 hours) and shorter ICU LOS (9.2 days vs. 19.1 days). The overall hospital stay of the guidelines group was also shorter (19.1 days vs. 34.3 days). The direct drug cost related to the implementation of the sedation guideline was also found to be lower (Mascia et al., 2000).

Post-Surgical Glycemic Control. Hyperglycemia was associated with mortality of ICU patients (Krinsley, 2008). The standard of tight glycemic control of critically ill patients became a standard of care following the Leuven Intensive Insulin Therapy Trial in 2001 (Van den Berghe et al., 2001). However, studies such as the NICE-SUGAR study have challenged the outcomes of this trial on survival of critically ill patients (Finfer et al., 2009). Marik and Preiser (2010) conducted a systematic review to identify the benefits and risks of tight glycemic control in ICU patients and to examine the variation in the outcomes among the reported trials. Prospective randomized controlled trials examining the impact of tight glycemic control $(80-110 \mathrm{mg} / \mathrm{dl})$ on mortality of ICU patients were included. A total of seven randomized controlled trials were identified and meta-analytic techniques were used to analyze the data. The trials included 11,425 patients. The results indicated that tight glycemic control did not reduce the28-day mortality, incidence of blood stream infections, or renal replacement therapy. Tight glycemic control patients were found to have higher incidences of hypoglycemia. Meta regression showed that there was a significant relationship between the 28-day mortality and proportion of calories provided parenterally. The authors suggested that the difference in outcomes between the Leuven Intensive Insulin Therapy Trials and the other studies could be due to the different use of parenteral nutrition. Excluding the two 
the Leuven Intensive Insulin Therapy Trials from the analysis, the mortality was lower in the control group. As a result, tight glycemic control was found to be associated with high incidence of hypoglycemia and increased death in patients not receiving parenteral nutrition (Marik \& Preiser, 2010).

Grey and Perdrizet (2004) investigated the impact of hyperglycemia in glucose intolerant patients without diabetes on nosocomial infections in a surgical ICU. A randomized controlled clinical trial at a 12-bed ICU with 61 critically ill patients requiring treatment of hyperglycemia (i.e., glucose values greater or equal to $140 \mathrm{mg} / \mathrm{dl}$ ) was conducted. Patients were randomized into standard insulin therapy (i.e., target range: $180-220 \mathrm{mg} / \mathrm{dl}$ ) and strict insulin therapy (i.e., $80-120 \mathrm{mg} / \mathrm{dl}$ ) groups. A total of 27 patients were in the standard insulin therapy group and 34 patients were in the strict insulin therapy group. The findings showed a significant decrease in the mean glucose level in the strict insulin therapy group. This decrease was associated with a reduced incidence of total nosocomial infections (i.e., intravascular devices, bloodstream, intravascular devices related to bloodstream, and surgical site). However, the hypoglycemia was significantly higher in the strict insulin therapy group (Grey \& Perdrizet, 2004). These findings suggested that strict insulin therapy may contribute to decreasing the incidence of nosocomial infections in nondiabetic patients in the surgical ICU. However, the implementation of strict insulin therapy in the ICU may always consider hypoglycemia as a potential complication. Hypoglycemia in ICUs has been associated with increased mortality. In a study to examine the relationship between hypoglycemia and mortality in ICUs, Hermanides and colleagues (2010) conducted a retrospective study with 5,961 patients. The findings indicated that the incidence of 
death among patients exposed to hypoglycemia was 40 per 1,000 ICU days compared to 17 per 1,000 ICU days in patients not exposed to hypoglycemia (Hermanides et al., 2010).

Krinsely (2008) investigated whether glycemic variability has an independent effect on mortality in a heterogeneous population of critically ill patients. In a retrospective review of a cohort of prospectively reviewed patients at a 14-bed ICU, 3,252 patients consecutively admitted to the ICU with at least three venous glucose samples were examined. The mean APACHE II was $20.0(\mathrm{SD}=8.9)$ and mortality was $24.4 \%$. The mean glucose level ranged from $70 \mathrm{mg} / \mathrm{dl}$ to $99 \mathrm{mg} / \mathrm{dl}$ in $35.9 \%$ of patients. The mean ICU LOS was 1.2 ( $\mathrm{SD}=.7)$. Patients were divided into four quartiles: 70-99 $\mathrm{mg} / \mathrm{dl}, 100-119 \mathrm{mg} / \mathrm{dl}, 120-139 \mathrm{mg} / \mathrm{dl}, 140-179 \mathrm{mg} / \mathrm{dl}$, and $180 \mathrm{mg} / \mathrm{dl}$ and above. There was a strong association between glycemic variability and mortality. The study findings indicated that mortality ranged from $5.9 \%$ in the first quartile to $30.1 \%$ in the fourth quartile. The lowest quartile was found to have the lowest mortality of $12.1 \%$. The ICU LOS was shorter among patients in the first quartile. As a result, the study findings suggested that lower glycemic variability may improve mortality and outcomes of ICU patients (Krinsely, 2008).

Krinsley and Jones (2006) assessed the effect of an intensive glycemia management protocol on the cost of care of critically ill adult patients. A total of 800 consecutive admissions to the ICU prior to the initiation of an intensive glucose management protocol were compared to the first 800 patients admitted to the ICU following the initiation of the protocol. The two groups were matched for age, gender, race, distribution of admitting diagnosis, prevalence of diabetes, and APACHE II scores. 
Approximately $40 \%$ of patients were mechanically ventilated during the baseline period and $33.6 \%$ during the treatment period. The ICU LOS decreased from 1.5 days to 1.3 day among patients not requiring mechanical ventilation and from 4.2 days during baseline period to 3.4 days during the treatment period among patients requiring mechanical ventilation. The mechanical ventilation days decreased from 2.0 days during the baseline period to 1.7 days during the treatment period. The cost during the two periods were compared for duration of ICU admission, duration of hospitalization after last ICU discharge, duration of mechanical ventilation, and resource use cost. The findings showed that the annualized adjusted total savings was $\$ 133,500$ and the mean adjusted cost savings per patient was $\$ 1,580$ (Krinsley \& Jones, 2006).

\section{Healthcare Charges}

Economic evaluation of resource use in hospitals is often reported as either microcosts or as charges (Scott, 2009). Micro-costs are the expenses incurred by hospitals in goods and services. In other words, a cost is derived for each step of an intervention (e.g., staff time, supplies, medications, and out-of-pocket expenses) (Smith et al., 2010). Micro-costs provide a more precise estimate of the economic value of the resources used in hospital care (Scott, 2009). The use of hospital charges to estimate cost may be imprecise due to cost-shifting or overestimation. Cost shifting practices are used frequently by hospitals to account for the non-reimbursable indigent care provided by the institution (Finkler \& Getzen, 2008). As a result, cost shifting may occur reimbursing some services at a higher rate than others within the same hospital. Hence, when evaluating studies on costs of hospital services it is necessary to make this distinction a priori to avoid erroneous interpretations. If micro-costing is not feasible, cost estimates 
based on adjusted costs or using cost-to-charge ratios like CMS (Centers for Medicare and Medicaid Services) may suffice.

It is crucial to recognize the costs of patient conditions (e.g., HAIs, pressure ulcer) and distinguish their impact on the micro-cost or the resources used for the treatment of these conditions and the hospital charges that are incurred from patient care when studying the economic burden of these patient conditions. Ideally, the most accurate analysis would be to use micro-costs since they reflect the actual cost of the services and treatments provided. Unfortunately, information on the micro-costs may not be feasible in most instances and therefore total hospital charges are used as an alternative in many studies and published reports. There are also intangible costs for patient conditions, costs that may have equal or greater burden than the financial costs. For instance, Scott (2009) defines these intangible costs as part of the social costs of HAIs, namely psychological cost (i.e., anxiety, grief, job loss, ensuing disabilities), pain and suffering, and change in social functioning and activities of daily living. There is a dearth of literature documenting these intangible costs as a result of HAIs and pressure ulcer in the ICU (Scott, 2009).

Given the magnitude of HAIs and pressure ulcers in the ICU it is almost impossible to quantify the costs of complications of each HAI or pressure ulcer episode. In fact, the number of complications that may result from an episode of HAIs is closely related to the nature of the entity but not limited to the primary target organ or systems. For example, a patient experiencing an episode of VAP initially may develop complications related to the respiratory system like Acute Respiratory Distress Syndrome but later may evolve into renal failure, septicemia, liver failure, cardiogenic shock, and 
ultimately multi-organ failure. Literature is devoid on the costs of complications of HAI episodes. The same is true for literature on the long term care costs from HAIs.

Healthcare Associated Infections and pressure ulcers can affect hospitals adversely. The economic impact of these conditions is significantly critical as the extra costs originated by HAIs are not matched with increased revenues when Medicare is the payer (Pyenson et al., 2007). This may also be true for some private insurers that tend to follow the Medicare reimbursement practices. This is particularly true when considering that the Centers for Medicare and Medicaid Services (CMS) will no longer reimburse additional expenses for specific conditions named never events. It is important to clarify the use of the term never events since the public and in some instances healthcare professionals have been misusing the term to refer to non-reimbursable occurrences under CMS guidelines.

Lembitz (2010) distinctively dispelled the misconceptions and misuse of the term neverevent wrongly associated with nonreimbursable serious hospital-acquired conditions (HACs) in a classic article to educate the medical community, specifically the membership of the American Association of Orthopedic Surgeons, about these incidents. Non reimbursable HACs under CMS guidelines were adopted to stimulate hospitals to develop safer practices and to restrict the billing of preventable complications to Medicare and Medicaid. Thus, the conditions listed under HACs are deemed preventable and their occurrence may have been curtailed by the use of evidence-based practices or guidelines. Included on the CMS list of nonreimbursable HACs are HAIs (e.g., CAUTI) and pressure ulcers. Healthcare-Associated Pneumonias, specifically VAP, may be included on the list of HACs in the near future. However, certain conditions listed as 
HACs may not be preventable (e.g., pressure ulcers). Sometimes a zero-incidence is unlikely and HACs may occur even when evidence-based practices and guidelines are instituted and the standard of care is implemented. This dichotomy allows for arguments when resubmitting denied claims for case revisions to determine preventability and ultimately payment on the claims. This fact may also be taken into consideration when assessing risk indexes for HAIs and pressure ulcers.

The impact of HACs on Medicare is important. HAIs and pressure ulcers in the ICU are more prevalent in Medicare patients since the elderly population is more vulnerable given their advanced age, the incidence of chronic illness associated with old age, the likelihood of needing hospitalization for exacerbation of chronic conditions, or need for surgery. Since Medicare is the largest single payer for most hospitals it is understandable that any factor affecting the Medicare-dependent population will be reflected in reimbursement of services by Medicare. Conversely, any financial constraint such as reimbursement failure or delayed reimbursement will negatively impact providers and hospitals financially and organizationally.

The Centers for Medicare and Medicaid (CMS) focused on two criterions to determine which conditions will result in a DRG with a higher payment if present as a secondary diagnosis, acquired after admission, and could have been prevented through application of evidence based guidelines (Kuper \& Septimus, 2009). These criterions for complications and comorbidities were high-cost and high-volume (Brown, Doloresco, \& Mylotte, 2009). As a result, hospitals will not be reimbursed from CMS if the HAI was listed as a secondary diagnosis and the condition was not listed as present on admission (Kuper \& Septimus, 2009). High-cost is based on the mean total charge for 
hospitalizations with the complications of interest (i.e., HAIs) and high volume is based on the increased incidence and prevalence of the complication of interest (i.e., HAIs) (Brown et al., 2009). In short, these changes to the CMS' reimbursement policies are 2fold: 1) additional reimbursement is not provided for any HAC contracted during the current hospitalization, and 2) hospitals will receive reduced payments if they fail to report these events (Brown et al., 2009).

CMS reported $\$ 3.6$ billion dollars in charges for hospitalizations with an HAI in 2007 (Centers for Medicare and Medicaid Services, HHS [CMS, HHS], 2008).

Economic expenses of these HAIs are expected to increase if appropriate preventative measures are not implemented. However, the clinical outcomes resulting from the recommendations of CMS and the consequent reimbursement issues lead to debates related to the resultant quality of care. Critics of the reimbursement changes argue that the changes discourage the provision of poor-quality care instead of providing incentives to provide high-quality care (Brown et al., 2009). Others favor the changes arguing that the way to incentivize quality care is to promote evidence-based practices and standardized care. Nevertheless, implementation of the changes in reimbursement from the federal government is aimed first to decrease CMS' expenses, to promote safer practices, improve quality of care, and reduce or eliminate preventable complications during hospitalizations, and decrease morbidity and mortality.

The 2010 Patient Protection and Affordable Care Act (ACA) contain provisions intended to reduce HAIs. In addition, financial penalties for hospitals with high-risk adjusted rates of HAIs are in place. The ACA provisions did contribute to the increased measurement and reporting of HAIs by all hospitals. As of 2015, hospitals in the top 
quartile of national HAI condition rates will receive almost one hundred percent of their applicable Medicare payments for all discharges based on DRGs (HHS, 2009). The American Recovery and Reinvestment Act of 2009 provided $\$ 50$ million in funding to support states in the prevention and reduction of HAIs. These funds are to be invested in efforts that support surveillance and research on HAIs, training of healthcare workers in prevention and measure outcomes, and to improve patient care (HHS, 2009).

In summary, there is great need to continue raising awareness of HAIs and pressure ulcers since the numbers are projected to increase and the financial cost to the nation is great. Education of providers and the public is critical in curtailing HAIs and pressure ulcers in the ICU. Studies reveal that education and training of healthcare workers increases compliance with best practices in preventing HAIs (Safdar \& Abad, 2008).

Research in this area is needed to assess effectiveness of ICU-wide protocol implementation on transmission prevention, developing strategies to measure these conditions (i.e., HAIs, pressure ulcers in ICU), evaluating post-discharge surveillance methods, and developing standardized methods for measuring and reporting prevention practices. Some of these recommendations have been suggested by the Department of Health and Human Services in their Action Plan to Prevent Healthcare-Associated Infections (HHS, 2009). At this time, no valid outcome or process metric has been identified for VAP (HHS, 2009). The benefits of prevention are clear. Scott (2009) reported benefits from $\$ 5.7$ to $\$ 6.8$ billion (20 percent of infections preventable, CPI for all urban consumers) to $\$ 25.0$ to $\$ 31.5$ billion (70 percent of infections preventable, CPI for inpatient hospital services) in ranges of effectiveness of possible infection control 
interventions. Healthcare-Associated Infections and pressure ulcers affect the country's economy and have transformed financial models of reimbursement at federal and state levels with private payers expected to change reimbursement practices as well. As a result there is a heightened sense of accountability and commitment to quality care growing among healthcare providers. The result is a change in health care practices resulting from changes in reimbursement.

Acquiring an infection through medical treatment continues to challenge the healthcare establishment. Approximately 2 million cases of Healthcare-Associated Infections (HAIs: infections associated with healthcare delivery in any health care setting) occur annually in hospitalized patients in the US, resulting in approximately 100,000 deaths and $\$ 17$ billion in additional healthcare costs (Jarvis, 2007; Parekh, 2008; Yokoe \& Classen, 2008). Patients admitted to intensive care units (ICUs) are at even greater risk for HAIs as a result of their medical condition and treatment that requires multiple invasive procedures such as central lines, indwelling catheters, mechanical ventilation, and others (Jegers et al., 2002). The economic impact of HAIs and who bears these costs will be examined in the following section.

\section{Reimbursement Models.}

Value-Based Purchasing Plan. The Centers for Medicare and Medicaid Services (CMS) have developed a value-based purchasing (VBP) initiative in an effort to enhance the quality and efficiency of the services provided to Medicare beneficiaries (HHS, 2009). This model intends to transform Medicare from a passive payer to an active purchaser of higher value healthcare services. Through the use of HACs, Present on 
Admission Indicator Reporting and Hospital Pay-for-Reporting initiatives CMS is promoting quality and efficient care.

Since October, 2008, Medicare will no longer assign an inpatient hospital discharge to a higher paying Medicare-severity diagnosis-related group (MS-DRG) if the selected condition listed on the claim was not present on admission. For this reason, since October of 2007 CMS began requiring hospitals to submit present on admission (POA) indicators on all Medicare claims (HHS, 2009). The POA will identify which conditions are HAC for payment purposes but also will yield great data for research purposes (HHS, 2009).

The VBP plan builds on the current hospital pay-for-reporting program and establishes performance-based Medicare hospital payment. A portion of the hospital payment will be contingent on actual performance rather than on a hospital's reporting of measurement data (HHS, 2009). The VBP performance measures will include infection rates.

The performance assessment model is the methodology used for scoring hospital performance on specific measures. Measures are the foundation of performance-based payment (HHS, 2009). To qualify for incentive payment the hospital must report an all measures relevant to its service mix such as quality domains that include clinical process of care, patient experiences of care, and others. The resulting aggregate scores will be used to determine the incentive payment. The model evaluates a hospital's performance on each measure based on the highest of either an attainment score or an improvement score (HHS, 2009). The improvement score will be determined by comparing the hospital's current score with its baseline performance. The source of the incentive 
payment will be a percentage of the hospital's base operating DRG payments. In effect, hospitals will earn back a portion of their Medicare payments by performing at a high level or improving their performance (HHS, 2009).

Powerful incentives derived from hospital VBP implementation are expected to curtail the incidence of HAIs and pressure ulcers. Financially, higher performing hospitals will receive larger payments. Publicly, the images of the higher performing hospitals will be reported and accessible to the public, encouraging institutions to compete for clients through improved performance. Inversely, the lower performing hospitals will receive smaller payments and will have to strive for public's confidence when reporting of performance results is negative. In the end, the public wins. They either will benefit from selecting higher performance institutions for care or will force the deficient ones to strive for delivering optimal care.

Inpatient Proposed Payment System Payment Incentives (IPPS). This system encourages hospitals to deliver quality and efficient care. Usually, hospitals receive the same payment for stays that vary in length of stay and the intensity of the services provided. This gives hospitals incentive to avoid unnecessary costs. Complications, including infections and pressure ulcers acquired in the hospital do not generate higher payments than the hospitals would otherwise receive for uncomplicated cases paid under the same DRG (HHS, 2009). The Centers for Medicare and Medicaid Services no longer reimburses over the typical IPPS rate for care required as a result of an HAI or pressure ulcer. Many private health insurance companies are following CMS and will no longer reimburse hospitals for HAIs and pressure ulcers. Information on private payment of HAIs and pressure ulcers by health insurance companies is not readily disseminated. 
Further research is required to evaluate the reimbursement practices of these private insurers.

Over ten years earlier Reynolds et al. (1988) evaluated the impact of critical care physician staffing (intensivists) on patients with septic shock in a medical ICU (MICU) at a teaching hospital. The study compared patients not followed by intensivists for 12 months (pre-intensivist interval) with patients followed by intensivists for 12 months (post intensivist interval) in the same medical intensive care unit. The Acute Physiologic and Chronic Health Evaluation scores (APACHE) used to compare severity of illness were similar for each group. The study found significantly lower mortality rates in the post intensivist interval. Interestingly, the study also found correlations between survival rates and costs. Sixty-two percent of the total charges of patients with septic shock in the pre intensivist interval were expended in patients that did not survive. The costs in nonsurvivor patients in the post intensivist interval were significantly reduced to $49 \%$ (Reynolds et al., 1988).

The increased healthcare costs of HAIs and pressure ulcers can be attributed to the emergence of complications that result in additional diagnostic procedures, extended medical management, and prolonged hospitalization. HAIs and pressure ulcers are a significant cause of increase in length of stay for hospitalized patients and consequently of the cost associated with that stay. These costs are directly related to the type of HAI, the resources needed to treat such infections, and the overall involvement of direct and indirect resource utilization during the course of these occurrences. The economic impact of these conditions on patients, hospitals, payers, and the overall burden on the healthcare system are substantial. Perhaps initiatives like mandatory HAI reporting laws 
in Colorado, Connecticut, Delaware, Illinois, Massachusetts, Maryland, Oklahoma, Pennsylvania, Tennessee, Virginia, and Washington (Edwards et al, 2009) will help decrease the incidence of HAIs and will promote transparency in care practices.

In addition to the significant morbidity and mortality resulting from HAIs, hospital-acquired HAIs represent $\$ 28$ to $\$ 33$ billion dollars in preventable healthcare expenditures yearly (HHS, 2009). Hospital care for HAIs cost Medicare an estimated \$324 million in October 2008 alone and extended on average a 19-day longer length of stay (Lucado et al., 2010). Annual data for cost to Medicare for HAIs is not available since Medicare is no longer reimbursing for preventable HAIs and medical errors since October 2008 as a result of U.S. Congress Deficit Reduction Act of 2005 (Kuper \& Septimus, 2009). Instead, data on related conditions to HAIs may be obtained from the Medicare Provider Analysis and Review (MEDPAR) File. The Medicare Provider Analysis and Review File publish data by DRGs from claims for services provided to Medicare beneficiaries admitted to certified inpatient hospitals. For example, the 2009 cost of septicemia with mechanical ventilation for 96 or more hours (i.e. e., related to VAP) was estimated to be 2.3 billion dollars (Centers for Medicare \& Medicaid Services, 2009).

\section{APN Intensivists Special Initiatives}

APN special initiatives have consisted of protocols and interventions such as surveillance, assessment, and management to decrease VAP, CAUTI, pressure ulcer, patient initiated removal of devices, and other patient outcomes. These interventions have been discussed in the previous sections of this chapter (see pages 55 to 94). Care provided by APNs has been documented in numerous studies examining quality of care 
(Kleinpell \& Gawlinski, 2005), morbidity and mortality (Gracias et al., 2008; Hoffman et al., 2005; Munro \& Taylor-Panek, 2007; Russell et al., 2002; Sole et al., 2001; Burns et al., 2003; Meyer \& Miers, 2005). The results of these studies indicate that the quality of care provided by APNs may be equal to physicians as well as cost efficient (Horrocks et al., 2002; Kinnersley et al., 2000). In addition, patient satisfaction data show the high quality care provided by APNs (Rudy et al., 1998). Furthermore, there have been efforts on patient-centered care (PCC).

A study designed by Sidani (2008) evaluated the contributions of APNs on patient outcomes. The effects of two components (patient participation in care and individualization of care) of patient-centered care (PCC) on patient's functional status, self-care ability, and satisfaction with care were examined to determine the extent to which APNs care was related to patient outcomes (Sidani, 2008). The author hypothesized that patient participation in care and individualization of care contributed to the selected outcomes. A repeated measures design was used to examine patients' perception of the extent to which APNs provide PCC components, and to explore the effects of PCC components on patient outcomes (Sidani, 2008). Data were obtained at three points in time: within 24-48 hours of admission to in-hospital unit, 1 week post discharge, and 6-8 weeks post discharge. This study was conducted at eight hospitals in two cities in Southern Ontario, Canada. The target population included patients admitted to acute care hospitals and assigned to the care of APNs (Sidani, 2008).

In discussing the findings, Sidani (2008) confirmed that patients were able to evaluate provision of different PCC components and indicated its association with outcomes. The author identified not only that patients are demanding an active role in 
care-related decision making but that individualized care is a critical attribute of highquality care as perceived by patients. Aside from the resulting implications of implementing PCC in acute care in terms of increased levels of satisfaction with care, this study illustrated the need for further research in this area. It will be interesting to examine if future research conducted in this topic will yield similar results when tested in the U.S. or in other countries.

A randomized controlled trial of NPs in neonatal intensive care compared a CNS/ NP team with a pediatric resident team in a 33-bed tertiary level neonatal ICU. A sample of 821 infants admitted to the NICU were randomized to care by a CNS/NP team $(n=414)$ or a pediatric resident team $(n=407)$. The outcomes monitored were mortality, number of complications, length of stay, quality of care, parents' satisfaction, long term outcomes, and cost. The findings revealed that the outcomes were similar between both teams. There was no difference in the death rates, complication rates, average length of stay, parental satisfaction with care, percentage of infants with developmental delay, or cost of care between the two teams. As a result, the use of APNs in the NICU as an alternative to pediatric residents in delivering care to critically ill neonates is cost effective and a safe choice (Mitchell-DiCenso et al., 1996).

In a similar study on adult patients, Rudy and colleagues (1998) compared the care activities and outcomes of patients cared for by ACNPs, physician assistants (PAs), and resident physicians on 289 ICU patients. Eleven ACNPs and five PAs and a group of matched resident physicians were studied over 14 months. Data on length of stay, inhospital mortality and readmissions rates on 187 patients cared for by ACNP/PA team and 202 patients cared for by resident physicians. The study results yielded similar 
outcomes for both groups although the ACNP/PA team was more likely to include patients' social history, to discuss patients with bed side nurses, and interact with patients' family. The ACNP/PA team was more likely to discuss patients with ICU nurses and the patient's family and spent more time in research and administrative activities. Resident physicians, however, cared for older and sicker patients, worked more hours, and spent more time on rounds (Rudy et al., 1998).

In the trauma unit, the role of NPs was also examined in a study by Spisso and colleagues (1990) at the University of California, Davis Medical Center. The study compared pre-NP (1985-86) and post-NP (1986-87) on cost of care, length of stay, documentation time, clinical wait time, and time savings for house physicians. The findings revealed that with the NPs practicing in the trauma team, there was a decrease in patient length of stay, outpatient clinic waiting times (41 to 19 minutes), and patient complaints (17 to 7 per year) in addition to an increase in quality of care documentation such as discharge summaries ( $95 \%$ complete by NPs versus $75 \%$ by residents) (Spisso et al., 1990). Again, NPs were shown to be effective members of the healthcare team in improving patient outcomes and decreasing cost.

Similarly, Sole and colleagues (2001) described the role of ACNPs and PAs at a level 1 trauma center. The focus of the study was to document the initial evaluation of patients (i.e., diagnosis) and changes in medical orders (i.e., changes in medication orders, consultations, and others) written by two nonphysician providers through a retrospective design using chart reviews. On a sample of 93 patients, the findings supported previous studies that reported that ACNPs tend to care for patients with complex and specialized needs. The study findings documented that nonphysicians 
providers can care for trauma patients after being stabilized and discharged from an ICU with similar outcomes as physicians (Sole et al., 2001).

In another randomized controlled trial, Pioro et al. (2001) compared resource use and outcomes of general medical patients receiving NP-based care and traditional house staff care. In a sample of 193 patients receiving NP-based care and 188 house staff care, patients had similar outcomes in terms of resource use (length of stay, total charges, and ancillary charges) and hospital charges. The study used a heterogeneous sample of general medical patients excluding patients in intensive care units or other specialty units (telemetry ward, coronary care step-down unit, hematology-oncology ward, bone marrow transplant ward, HIV ward), and those transferred from intensive care units. These findings confirmed that the care delivered by NPs supported by attending physicians has similar clinical and functional outcomes as that delivered by house staff supported by attending physicians (Pioro et al., 2001).

At the University of Massachusetts, McMullen, Alexander, Bourgeois, and Goodman (2001) studied the one year outcomes of ACNPs as compared to the traditional physician care provided at an acute care unit at the medical center. A sample of 405 patients from the traditional service and 296 from the Nurse Practitioner Attending Collaborative Service (NPACS) were recruited over the year. The outcomes were patient health (Functional Health Status Short Form), patient satisfaction, physician satisfaction, and staff satisfaction. The study findings indicated that patients with the traditional service scored lower (i.e., less healthy) than the NPACS patients on admission $(\mathrm{p}<.001)$ and one month after discharge $(\mathrm{p}=.03)$. The findings indicated significant difference 
between the two patient groups in the categories of patient satisfaction where patients indicated increased satisfaction in the NPACS group (McMullen et al., 2001).

In evaluating ACNP clinical and financial outcomes for neuroscience patients, Russell et al. (2002) used an "outcomes management" model using ACNPs to manage and monitor 122 adult patients in a neuroscience ICU or acute care neurosurgery unit. The outcomes management model of care uses APNs to manage and monitor patients assigned to a multidisciplinary plan of care (Russell et al., 2002). Patient outcomes were compared between the pre-implementation and post-implementation phases. The findings revealed that patients had shorter overall length of stay $(\mathrm{p}=.03)$, shorter mean length of stay in ICU $(\mathrm{p}<.001)$, lower rates of urinary tract infections $(\mathrm{p}<.05)$, skin breakdown $(\mathrm{p}<.05)$, shorter time of urinary catheter discontinuation $(\mathrm{p}<.05)$, and mobilization $(\mathrm{p}<.05)$ in the post implementation phase. The outcomes-managed group had hospitalized saving of $\$ 2,467,328$ (Russell et al., 2002).

Hoffman and colleagues (2003) studied the role of ACNPs in the ICU and critical care units and expanded the body of knowledge in comparing ACNP and physician outcomes. A causal comparative longitudinal study was conducted at three time points when $\operatorname{ACNP}(\mathrm{n}=1)$ had 6 months or less experience in the role (T1), after 12 months experience in the role $(\mathrm{T} 2)$, and when physicians in training $(\mathrm{n}=6)$ provided care on a rotational basis in the absence of an ACNP (T3). The study was conducted at a 6-bed step down medical ICU. No significant differences in the number of patients managed per day by the ACNP $(\mathrm{M}=5.9, \mathrm{SD}=0.3)$ and the physicians in training $(\mathrm{M}=5.8, \mathrm{SD}=0.6)$ or the number of patients per day being weaned from mechanical ventilation ( $\mathrm{M}=2.2$, $\mathrm{SD}=1.4$ for $\mathrm{ACNP}, \mathrm{M}=2.3, \mathrm{SD}=1.3$ for the physicians in training) were found. Mean 
scores on the Chronic Health Evaluation III on the first day of admission to the SDMICU were high for patients managed by the $\operatorname{ACNP}(\mathrm{M}=51, \mathrm{SD}=16)$ and physicians in training $(\mathrm{M}=51, \mathrm{SD}=25)$ but were similar to scores for the first day of admission for other ICUs in the same facility $(\mathrm{M}=49, \mathrm{SD}=6)$. The comparison between $\mathrm{T} 1$ and $\mathrm{T} 2$ yielded similar findings. But, when comparing the ACNP outcomes with the physicians (T2 and T3), there was a significant difference in the time spent by ACNPs in coordinating care ( $p$ $<.001)$ and less time in non-unit activities $(\mathrm{p}<.001)$. The ACNPs had no significant differences in routine management of patients, coordination of care, and non-unit activities regardless of the length of experience (less than 6 months versus more than 12 months). In comparing the ACNP and physician in training, results showed that the ACNP spent more time in activities related to coordination of care $(45 \%$ versus $18 \%, p<$ $.001)$ and less time in non-unit activities $(15 \%$ versus $37 \%, \mathrm{p}<.001)$. Physicians instead spent more time off the unit in activities related to their training $(\mathrm{p}<.05)$. The study concluded that the ACNP and physician in training had similar efficiency in performing the required tasks whereas the ACNP spent more time with patients and families and collaborating with other healthcare team members (Hoffman et al., 2003).

Similarly, Hoffman et al. (2005) compared outcomes in a subacute medical ICU of patients managed by ACNPs and a physician attending (group 1) or a critical care/pulmonary fellow and physician attending (group 2). During a 31-month period, 526 patients admitted to the unit were managed by one of the two teams and were compared for a variety of outcomes (i.e., length of stay in the ICU, number of mechanical ventilation days, reintubation rates, weaning status, disposition site, readmission rates, and number of deaths). The results showed that the patients managed by ACNPs had 
more comorbidities $(\mathrm{p}=.02)$ indicated by the Charlson Comorbidity Scores. There was no difference between the two teams in terms of readmission rates within 72 hours of discharge from the subacute medical ICU. The patients managed by the teams did not differ by work load or medical condition. There was also no significant difference in readmission to a high acuity unit $(\mathrm{p}=.25)$, mortality $(\mathrm{p}=.25)$, weaning trials $(\mathrm{p}=.42)$, length of stay, duration of mechanical ventilation $(p=.18)$, weaning status at time of discharge from the unit $(\mathrm{p}=.80)$, and disposition $(\mathrm{p}=.28)$. Patients managed by fellows had significantly more reintubations. The two teams were found to produce similar outcomes (i.e., length of stay in the ICU, number of mechanical ventilation days, reintubation rates, weaning status, disposition site, readmission rates, and number of deaths with no significant differences in the care provided (Hoffman et al., 2005).

Examining the impact of ACNP interventions on patient outcomes post discharge from the ICU, Daly, Douglas, Kelley, O’Toole, and Montenegro (2005) tested the outcomes of a Disease Management (DM) program on hospital readmissions and cost. The randomized control trial was conducted on 334 patients from one academic medical center that underwent more than three days of mechanical ventilation and were discharged home. The experimental group $(n=231)$ received care coordination, family support, teaching, and monitoring of therapies from a team of APNs, geriatrician, and a pulmonologist for 2 months post hospital discharge. Patients receiving DM services had significantly fewer mean days of rehospitalization as compared to the control groups. The cost effectiveness was calculated using the number of reduced hospital days and cost of APNs (i.e., salary plus benefits). In the intervention group, there were a 6.31 fewer days of hospitalization and a decreased hospital charge of around $\$ 3,415$ per day or 
$\$ 21,548.65$ per patient. The savings were assumed for the 93 readmitted intervention patients and were estimated to be $\$ 2,004,024$. The cost savings were around $\$ 481,811$ for those readmitted $(n=93)$ to the hospital. These findings are essential in light of the high cost of hospitalization and the resultant patient burden and outcomes.

Kleinpell and Gawlinski (2005) indicated that ACNP participants recognize the advantage of being an ACNP in positively affecting patient outcomes (i.e., length of stay, complications, costs, and readmission rates). Participants reported positive outcomes with reduced length of stay, complications, costs, and readmission rates; increased adherence to best practices, patients' access to care, patients' satisfaction, and education of patients, patients' family members, and staff; and better medical management, resource utilization, and continuity of care. These statements have been consistently supported by research data examining the effects of ACNPs on patient outcomes. Although there are a discrete number of studies focusing on ACNPs, research data indicates that ACNPs can provide quality and cost-effective care.

In summary, results of studies conducted on APN effects on patient outcomes, healthcare costs, and quality care are comparable with the results of studies done on APNs in primary care settings. The positive effects of APNs on patient outcomes, healthcare costs, and quality care in primary care are well supported in the literature; quality care and patient outcomes on HIV patients (Aiken et al., 1993), effects of APN intervention and cost (Brooten et al, 2002), effects of APNs reducing healthcare costs and improving pregnancy and infant outcomes in women, infants, and elders (Brooten et al, 2001, 2003; Naylor et al., 1999, 2004), and care delivery comparable with physician care (Horrocks et al., 2002; Kinnersley et al., 2000; Mundinger et al, 2000). 
Advanced Practice Nurses improve outcomes for patients in a variety of clinical settings. APNs care may result in decreased length of stay (Burns et al., 2003; Meyer \& Miers, 2005; Russell et al., 2002), decreased costs of care (Burns et al., 2003; Meyer \& Miers, 2005; Russell et al., 2002), decreased rates of urinary tract infection and skin breakdown (Russell et al., 2002), compliance with clinical practice guidelines, including deep vein thrombosis/ pulmonary embolus prophylaxis, stress bleeding prophylaxis, and anemia (Gracias et al., 2008), management of patients receiving mechanical ventilation (Hoffman et al., 2005), enhanced communication and collaboration (Vazirani, Hays, Shapiro, \& Cowan, 2005), continuity of care (Hoffman, Happ, Scharfenberg, DiVirgilioThomas, \& Tasota, 2004), improved outcomes in neonatal intensive care (MitchellDiCenso et al., 1996), cardiac surgery patients (Munro \& Taylor-Panek, 2007), and trauma patient care (Sole et al., 2001).

Despite the available data pointing to improved patient outcomes with the use of ACNPs in critical care only selected aspects of the practice have been examined and documented. Kleinpell (2005) reported that surveyed ACNPs respondents significantly increased the assessment of practice outcomes. Failure to publish and disseminate the results of those efforts contributes to the limited available research focused on the role of ACNPs, especially when various types of practice settings and specialty roles exist.

In the discussion of findings and implications of the Report to Congress, the need for future research areas were considered, specifically the critical care training of other healthcare providers within the medical model and other specialties (USDHHS-HRSA, 2006). However, despite all available data supporting the sustainability of the APN roles and their positive effect on patient outcomes and care costs, APNs were excluded as an 
alternative care model. Furthermore, it is interesting to note that the inclusion of ACNPs trained in acute or critical care, was not considered as a viable alternative to manage critically ill patients in ICUs or other critical care areas affected by the current physician intensivists' attrition. As far as nursing alternatives the only recommendation considered to alleviate this problem was perhaps improving the organizational nursing staff (USDHH-HRSA) without mention of the role of APNs within the nursing organizational context.

The current literature documents the need for further research into alternative methods of healthcare delivery in critical care. Further research is needed on the use of APNs, specifically APN Intensivists in the ICUs, to generate outcome-driven care indicators, demonstrated reductions in length of stay, decrease in costs, and prevention of healthcare-associated complications. The empirical evidence of the effect of APN Intensivists on patient outcomes remains scarce compared to the well documented effects of APNs in other areas and models of healthcare delivery. In light of this dearth of the literature examining the effects of APN Intensivists on patient outcomes, is needed to fill this gap in our knowledge. 


\section{CHAPTER III}

\section{Methodology}

\section{Research Design}

Using retrospective chart review, the primary aim of this study was to examine the dose effect of APN Intensivists in a surgical intensive care unit (SICU) on patient outcomes, healthcare charges, charges for APN Intensivists services and APN Intensivists special initiatives when APN Intensivist staffing differed. The study examined whether there was a difference in patient outcomes (HAIs [i.e., VAP, CAUTI], pressure ulcers, SICU length of stay [LOS], patient-initiated accidental removal of devices [i.e., selfextubation, removal of venous access, removal of urinary catheters, and removal of feeding tubes], sedation management, post-surgical glycemic control, and ventilator days and ventilator weaning), when the SICU is staffed by different APN Intensivists staffing levels. The study also examined if there was a difference in health care charges for SICU length of stay and charges for APN Intensivists services when the SICU is staffed by differing APN Intensivist staffing levels. Lastly, the study aimed to examine if there was a difference in the frequency of APN Intensivist special initiatives conducted over the four time periods during the 3-year study period between 2009 and 2011.

\section{Setting}

Jackson Memorial Hospital (JMH), the setting for this study, is a 1500-bed metropolitan county hospital serving the poor and underserved located in Miami-Dade County, Florida. The 40-bed SICU in this tertiary teaching hospital served an average of 800 patients each year who require intensive care and close monitoring after surgery in one of JMH's 35 operating rooms. Patients (> 18 years old) were admitted to the JMH 
SICU after surgical procedures that include vascular, open heart, biliary, pancreatic, maxillofacial, plastic, colorectal, gastrointestinal, bariatric, neurosurgical, orthopedic, genitourinary surgery, organ transplantation, and trauma overflow.

These immediate post-surgical adults were usually endotracheally intubated (nasally or orally) for respiratory compromise resulting from either surgery or postsurgical events. These patients usually came to the unit with central venous catheters having been inserted in the operating room or in the unit during the patient's stay by either the physicians or the APN Intensivists. A great number of these patients were intubated and mechanically ventilated, and or had central vascular access, and or had bladder catheters, or a combination of these three. The usual length of stay varied depending on a multitude of factors such as baseline physiological status, comorbidities, the nature of the surgical procedure, and whether patients experienced post-operative complications or not. Some of the more common complications were: hypovolemia, bleeding, acute renal failure, pulmonary embolism, shock (cardiogenic, septic, and/or hypovolemic), sepsis, deep-vein thrombosis, ventilator associated pneumonia (VAP), central-line associated blood-stream infections (CLABSIs), catheter-associated urinary tract infections (CAUTIs), and in worse cases multi-organ failure.

\section{Staffing}

Jackson Memorial Hospital SICU was staffed with approximately 110 critical care staff nurses total. An average of 15 critical care registered nurses were assigned per each 12-hour shift and provided nursing care for one or two patients, based on each patient's level of acuity. The SICU multidisciplinary team that provided care included attending physicians, surgical and anesthesia fellows, residents, interns, APN Intensivists, 
pharmacists, nutritionists, respiratory technicians, and physical and occupational therapists. During the study years nursing staffing may have differed.

Medical Staffing. Attending surgeons and anesthesiologists were physically present during the day and conducted morning rounds. They also provided coverage for the unit by phone, providing 24 hours of medical supervision to junior medical personnel and APN Intensivists until the following morning's rounds. Two or more fellows rotated into the JMH SICU for a 1-month period each. Their daily shift takes place from 07:00 to 21:00 with at least two 24-hour shifts during their rotation. A 3rd-year surgical resident rotated to the JMH SICU for two consecutive months, covering the unit from 21:00 to 11:00 during their weekly rotation for a maximum of 40 hours per week. Two to four different medical interns rotated to the unit each month.

Previously, and during the study years 2009 through 2010, the medical interns' rotation was different and staffing patterns differed. For the years 2009 to 2010 medical interns rotated alternating between day and night shifts for periods of 18 to 24 hours, allowing for 24-hour coverage of the unit by at least one intern at all times. Since 2011, medical residents rotated to the unit for shifts of 14 hours per day for a week, alternating between night and day coverage.

APN Intensivist Staffing. In a collaborative practice with physicians, APN Intensivists functioned autonomously and were responsible for the management of the JMH SICU patients' care, including: a) medical treatments and invasive procedures (e.g., endotracheal intubation and bedside tracheostomies, insertion of central venous and arterial lines, insertion of peripherally inserted central venous catheters, thoracotomy tubes, percutaneous drainages, distally placed small bowel feeding tubes), b) respiratory 
management (e.g., mechanical ventilators), c) data monitoring and surveillance (e.g., antibiograms, postoperative glycemic control measures), d) interpretation of diagnostic procedures (e. g., x-rays, computerized tomography [CT] scans, and magnetic resonance imaging [MRI]), e) teaching and training nursing and medical staff, and f) research. APN Intensivists were also responsible to set-up and maintain unit-specific databases (e.g., pressure ulcer incidence and prevalence, safety and quality indicators for cardiothoracic postoperative patients, VAP, and CAUTI, and infection control) for monitoring and surveillance of indicators and outcomes.

APN Intensivist staffing for the study period follows: For 2009, there were at least two APN Intensivists per shift per day (i.e., 24/7). For the first half of 2010 (i.e., January through June), there was at least one APN Intensivist per shift per day (i.e., 24/7). For the second half of 2010 (i.e., July through December) there was one APN Intensivist per shift but not for seven days. For 2011 there was zero to one APN Intensivist per day but not for seven days.

\section{Sample}

The sample for this study consisted of post-operative patients admitted to Jackson Memorial Hospital SICU between the years 2009 through 2011. Approximately more than 1,200 charts met the criteria during the study period. Random selection of charts was done for each time-period of the study (i.e., T1, T2, T3, and T4). Patient acuity was not used to match patients during sampling. Patient acuity was going to be determined using the Acute Physiology and Chronic Health Evaluation (APACHE) II scoring (Knaus, Draper, Wagner, \& Zimmerman, 1985) calculated by the Principal Investigator (PI) once the chart was selected from the chart pool. The APACHE II scoring system is 
an index of illness severity used for patient outcome prediction and is also used for assessing ICU performance and quality of care (Polderman, Girbes, Thijs, \& Strack van Schijndel, 2001). For the purpose of this study all APACHE II scores were scored by the PI since the JMH SICU does not document APACHE II scores on their patients. It was estimated that approximately 500 charts out of the 800 annual admissions to the SICU met the inclusion criteria.

Inclusion Criteria. Inclusion criteria were: 1) Medical records that indicated that the patient was initially admitted to the JMH SICU within the established period of time, 2) the patient was an adult (older than 18 years old) post-surgical patient, 3 ) the type of surgical procedure was included in the admission note, 4) primary diagnosis leading to surgical procedure was documented in the chart, 5) the patient was endotracheally intubated (orally or nasally), 6) the patient had an indwelling urinary catheter, 7) the patient was discharged from the intensive care unit to a regular hospital floor, and 8) the patient did not die in the SICU.

Exclusion Criteria. Medical records of the JMH SICU patients who are less than 18 years old will be excluded to avoid inclusion of pediatric patients that may be medically considered adults based on weight parameters. Excluding patients less than 18 years old will eliminate potential biases due to different physiological responses from those of adult populations. Other exclusion criteria were: 1) missing operative note regarding the surgical intervention, 2) missing primary diagnoses in the admission note, 3) patients admitted to the JMH SICU for other than post-surgical procedures, 4) trauma patients admitted initially to the JMH SICU as overflow, 4) surgical patients admitted to JMH SICU 24 hours after their initial surgery to avoid confounding factors attributed to 
care delivered outside the ICU setting, 5) JMH SICU patients without endotracheal tubes, and/or indwelling urinary catheters.

Power Analysis. For 2009 through 2011, a random sample of 204 JMH SICU patient charts per each time period (total $\mathrm{N}=816$ ) that met the inclusion and exclusion criteria were selected for review. Each of the four groups represented a different level of APN Intensivist staffing. Power was calculated with a total $\mathrm{N}$ of 204 for research questions 1 through 3. For research question 1, Chi Square and $t$-test analysis with a sample of 200 per group, medium effect size and alpha of 0.05 provided $>85 \%$ power. For research question 2, $t$-test analysis with a sample of 200 per group, medium effect size and alpha of 0.05 provided $>85 \%$ power. For research question 3, one-way ANOVA analysis comparing four groups (i.e., T1, T2, T3, and T4) with a sample of 204 per group, medium effect size and alpha of 0.05 provided $>85 \%$ power.

\section{Data Collection Procedures}

Following IRB approval from Florida International University and Jackson Memorial Hospital/University of Miami, 204 SICU patient charts were selected randomly for each of the four time periods 2009 through 2011, which resulted in a total of 816 charts in 4 groups with differing APN Intensivist staffing. The Jackson Memorial Hospital (JMH) Quality and Patient Safety Office Information Technology department generated the list of patients who were admitted to the SICU during each of the four time periods. The Principal Investigator (PI) and research assistants (RAs) then identified the charts that met the inclusion criteria for the study. From the resulting list, the PI randomly selected 204 patient charts for each time-group from a total of 816 using a table of random numbers with a random starting point. Data from the selected patients' charts 
were collected by PI and RAs on standardized data abstraction forms. A code book of definitions, criteria for specific diagnoses, and preferred sources of specific data within the chart were used with each chart review to enhance consistency of the data collection. Inter-rater reliability was conducted by randomly selecting $10 \%$ of charts from each group for re-abstraction by the PI and comparison with the original chart review data collected by the Research Assistant. A minimum inter-rater reliability of $85 \%$ was maintained. If RAs were not available and data were collected by the PI only, intra-rater reliability was calculated by randomly selecting $10 \%$ of charts from each group that were reviewed two months before. After the sampling, the JMH Finance Department was contacted to obtain the billing for each selected patient's stay. Total charges billed for the hospitalization, including charges billed by the APN Intensivists during the selected patient stay, and for the JMH SICU stay was requested from the JMH Finance Department to be recorded by the PI.

\section{Measures}

\section{Independent Variables}

Patient Demographics. Patient demographics included: age, gender, race and ethnicity, primary diagnosis, type of surgery, admission diagnosis, chronic illness, comorbidities, medications, smoking, drinking, use of illegal drugs or any other type of substance abuse, and source of insurance.

APN Intensivists Demographics. Age, gender, race, ethnicity, level of education, certification, years of experience as APN, years of experience as staff nurse, and years of experience in ICU as APN Intensivist were collected for descriptive purposes. 
APN Intensivists Staffing. Data on the number of APN Intensivists (per day and per shift) in each time-period between 2009 to 2011 were obtained from the JMH Nursing Administration.

Unit Staffing. Data on unit staffing (number of registered nurses, nurse: patient ratios) during the years 2009-2011 were to be obtained from the JMH nursing administration. These data were to be used to describe the unit staffing during the identified time-periods.

Mechanical Ventilation. Data on total time of endotracheal intubation, type of endotracheal tube, placement (oral or nasal) of endotracheal tube, tracheostomy, ventilator settings, number of unplanned extubations, and any re-intubations were collected from chart review.

Indwelling Bladder Catheter. Data on whether the patient had an indwelling bladder catheter at home prior to SICU admission; urinary tract infection on admission; placement or replacement of bladder catheter peri- or post-operatively, and documented catheter care were collected from chart review.

Level of Acuity. To control for level of acuity, data on level of patient acuity were to be collected using Acute Physiology and Chronic Health Evaluation (APACHE) II scores. Acute Physiology and Chronic Health Evaluation (APACHE) II is an index of severity of illness classification system revised from the original prototype APACHE. It is used to predict risk of death and is usually used as an instrument in studies to collect data about the index of severity of illness during hospitalizations. APACHE scores are calculated from 12 physiological measurements and two disease-related variables including age, heart rate, respiratory rate, mean arterial pressure, body temperature, 
fraction of inspired oxygen $\left(\mathrm{FiO}_{2}\right)$, arterial oxygenation $\left(\mathrm{PaO}_{2}\right)$, arterial $\mathrm{PH}$, Glasgow coma score, sodium levels, potassium levels, hematocrit, creatinine, and white blood cell count. APACHE II scores are usually calculated within the first 24 hours of ICU admission and are useful to classify ICU patients (Knaus et al., 1985). In this revised version of the APACHE scoring system (i.e., APACHE II), physiologic measurements were reduced from 34 in the original prototype to 12 , with a maximum possible score of 71, although Knaus et al. (1985) reported that scores rarely exceed 55 . The ability to classify patients by severity provides researchers and clinicians with a tool to improve treatment of critically ill patients. Therefore, APACHE II has been useful in clinical trials and in non-randomized studies to help investigators discern similarities and differences between control and experimental groups, in studies of specific disease groups, and for determining the relative benefit of an invasive procedure (Knaus et al., 1985).

Despite the widespread use of the APACHE II in medical research protocols, very little has been reported regarding reliability and validity and instead a wide inter observer variation in the application of the scoring has been observed (Chen, Martin, Morrison, \& Sibbald, 1999; Polderman et al., 2001; Polderman Thijs, \& Girbes, 1999). The majority of the scoring errors in tallying APACHE II scores have been observed in the Chronic Health category points, disregarded data inconsistent with patients' general physiological trends, and definition of what constituted the first 24 hours of ICU (Polderman, Girbes, et al., 2001). In light of these inconsistencies, Polderman and colleagues (2001) proposed that researchers using APACHE II scores should report their scores revealing if they were revised at the time of data collection and if not how and by whom these scores were 
calculated to render the scores reliable. The detailed description of the APACHE II scoring process increased reliability by decreasing the questioning of how and by whom those scores were obtained (Polderman et al., 2001).

\section{Dependent Variables}

Ventilator-Associated Pneumonia (VAP). VAP is a healthcare-associated pneumonia that is seen in patients using mechanical ventilation within 48 hours of intubation (early onset) or during the weaning period (late onset). VAP is diagnosed by new or progressive infiltrates on chest radiographs, positive sputum cultures for pathogens, fever $\left(>38^{\circ} \mathrm{C}\right)$, and increased white blood cell (WBC) count. Diagnosis of VAP was collected from recorded chart data on a standardized data abstraction form.

Catheter-Associated Urinary Tract Infection (CAUTI). CAUTI is a healthcareassociated infection seen in patients using indwelling bladder catheters. CAUTI is diagnosed by fever $\left(>38^{\circ} \mathrm{C}\right)$, positive urine cultures for pathogens, and increased WBC count. Diagnosis of CAUTI was examined in each chart selected and data recorded on a standardized data abstraction form.

Pressure Ulcers. Pressure ulcer is a skin area where tissue breakdown or necrosis has developed as a result of compression against a bony prominence or an external period. Diagnosis of unit acquired Pressure Ulcer, location, grade, was collected from recorded chart data on a standardized data abstraction form.

SICU Length of Stay (LOS). The SICU LOS was considered as the 24-hour calendar days from admission to discharge. SICU LOS was collected from recorded chart data on a standardized data abstraction form. Data collected included total hospital stay (before and after the SICU admission) and SICU LOS. Data were collected from 
admission to the hospital or to JMH SICU to discharge from the hospital or JMH SICU in calendar days. The date and time of admission was recorded.

Patient-initiated Accidental Removal of Devices. Patient initiated accidental removal of devices (i.e., self-removal of endotracheal tubes, vascular devices, indwelling bladder catheters, and feeding tubes) is the premature and unplanned removal of these devices. Data were collected from recorded chart data on a standardized data abstraction form that included the number of self extubations, removal of vascular devices, indwelling bladder catheters, and feeding tubes. Data were recorded as "yes" or "no" for each of the above and the number and type of removals for each patient.

Sedation Management. Sedation management is the use and titration of sedatives to address agitation and achieve specific sedation levels in ICU patients. Data were collected from recorded chart data on a standardized data abstraction form and included the sedation management protocol, whether it was in place for each patient or not and if sedation vacation (reduced or absent) was attempted.

Post-surgical Glycemic Control. Post-surgical glycemic control is the management of glucose levels to less than $200 \mathrm{mg} / \mathrm{dL}$ in the first 48 postoperative hours. Data was collected from recorded chart data on a standardized data abstraction form and included the glycemic control protocol and whether it was implemented or not and if glycemic goals (glucose levels of less than $200 \mathrm{mg} / \mathrm{dL}$ for the first two post-operative days) were met.

Ventilator Weaning. Weaning is the process of liberating patients from mechanical ventilation resulting in the removal of the endotracheal tube. Data were collected from recorded chart data on a standardized data abstraction form and included 
whether weaning was attempted and if it was successful. The number of weaning attempts was also recorded.

Ventilator-days. Ventilator day was considered as the 24-hour calendar day in which the patient received mechanical ventilation. Data were collected from the JMH SICU Respiratory Department monthly report data on a standardized data abstraction form and included total of ventilator-days for that month which was reported as an aggregate measure.

Indwelling Bladder Catheter Days. Indwelling catheter days was considered as the 24-hour day in which a patient had an indwelling bladder catheter. Data were collected from recorded chart data on a standardized data abstraction form and included the number of days that the patient had an indwelling bladder catheter.

Healthcare Charges for SICU and APN Intensivists Services. Total healthcare charges for SICU patient stays for the sample during each of the three study periods and total charges of APN Intensivists services were collected for each of the three study periods.

APN Intensivists' Initiatives. For each of the three study periods, data were collected on major APN Intensivists initiatives such as new protocols, development of databases for quality outcomes and safety patient indicators. Data were collected for initiatives from stored databases available to APN Intensivists' on a separate computer drive in the JMH SICU (i.e., V-Drive). 
Table 1

Study's Variables, Measures, and Data Sources

\section{Variable}

HAIs (VAP, CAUTI)

Pressure Ulcers

JMH SICU Length of Stay

Patient-initiated Removal of Devices

Sedation Management

Post-surgical Glycemic Control

Ventilator-days

Ventilator Weaning

Healthcare Charges for JMH SICU LOS

Charges for APN Intensivists Services
Measure

Onset \& duration of each event

Onset, location, grade, treatment, and evolution

Admission to discharge data will be collected recording date and time of admission and discharge, then converted to 24 -hour days

Numbers and dates of selfremoval of devices (i.e., endotracheal tube, vascular devices, indwelling bladder catheters, feeding tubes)

Sedation protocol in place

Post-surgical glycemic control protocol in place

Aggregate data

Successful extubation and number of weaning attempts

Healthcare charges billed for hospitalization during the JMH SICU LOS

Charges billed by APN Intensivists during the JMH SICU stay

Data on APN Intensivist initiatives (i.e., new protocols, development of databases for quality outcomes, patient safety indicators)
APN Intensivist Special Initiatives
Data Source

Chart abstraction

Chart abstraction

Chart abstraction

Chart abstraction

Chart abstraction

Chart abstraction

JMH SICU Respiratory Department monthly report

Chart abstraction

Financial Department at JMH

Financial Department at JMH

APNs database 


\section{Data Management}

Data management procedures are those that insure integrity during data collection and those that maintain integrity during coding and entry. Strategies used to ensure data integrity during data collection included: 1) identification number on all form pages, 2) data collection forms assembled and placed in envelopes ahead of time. All coding decisions were recorded in a codebook to ensure consistency in decision-making. Data were entered for analysis into Statistical Package for the Social Sciences 21 (SPSS ${ }^{\circledR}$ ) by the PI.

\section{Data Analysis}

Research Question 1 was addressed with Chi-square tests and $t$-tests. Research Question 2 was addressed with $t$-tests. Research Question 3 was analyzed using One-way ANOVA.

Table 2.

Research Questions Variables and Statistical Analysis

\begin{tabular}{|c|c|c|}
\hline Research Questions & Variables & Statistical Analysis \\
\hline $\begin{array}{l}\text { Was there a difference in patient } \\
\text { outcomes (HAIs [i.e., VAP, CAUTI], } \\
\text { pressure ulcers, SICU length of stay }\end{array}$ & $\begin{array}{l}\text { VAP \& CAUTI = onset } \\
\text { (dichotomous) \& duration } \\
\text { (continuous) }\end{array}$ & \\
\hline $\begin{array}{l}{[\mathrm{LOS}], \text { patient-initiated accidental }} \\
\text { removal of devices [i.e., self- }\end{array}$ & PU (dichotomous) & \\
\hline extubation, removal of venous & SICU LOS (continuous) & \\
\hline $\begin{array}{l}\text { access, removal of urinary catheters, } \\
\text { and removal of feeding tubes], } \\
\text { sedation management, post-surgical }\end{array}$ & $\begin{array}{l}\text { Self extubation } \\
\text { (dichotomous) }\end{array}$ & \\
\hline $\begin{array}{l}\text { glycemic control, and ventilator days } \\
\text { and ventilator weaning), when the } \\
\text { ICU is staffed by: }\end{array}$ & $\begin{array}{l}\text { Removal of CVC/IV/AL } \\
\text { (dichotomous) }\end{array}$ & \\
\hline
\end{tabular}


Table 2

Research Questions Variables and Statistical Analysis (continued)

Differences in patient outcomes (continued)
Removal urinary catheter

(dichotomous)

Removal of feeding tube

(dichotomous)

Sedation management

(dichotomous)

Glycemic control

(dichotomous)

Ventilator days (continuous)

Ventilator weaning

(dichotomous)

Indwelling Bladder catheter days (continuous)

Compared outcomes of the $2 \quad \chi^{2}$ (dichotomous) groups $t$-test (continuous)
Two APN Intensivists per
shift 24/7 (around the clock) compared to zero to one APN Intensivist per day (2009 [T1] vs. 2011 [T4])

One APN Intensivist per shift compared to zero to one APN Intensivist per day (January to June 2010 [T2] to 2011 [T4])

One APN Intensivist per shift but not for seven days compared to zero to one APN Intensivist per day (July to December 2010 [T3] to 2011 [T4])

Was there a difference in health care charges for SICU length of stay and APN Intensivists services when the ICU is staffed by:

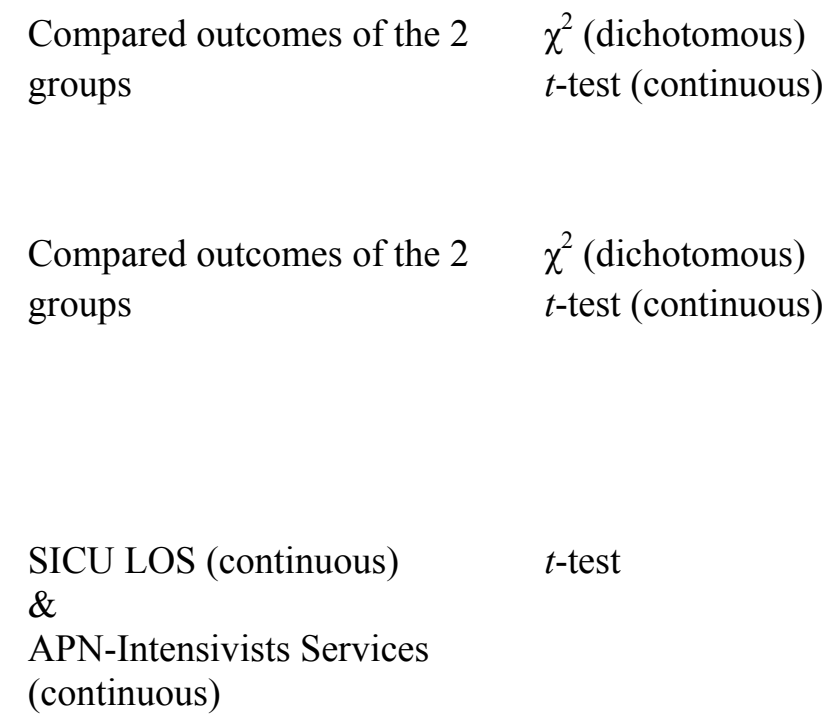


Table 2

Research Questions Variables and Statistical Analysis (continued)

[T1] vs. 2011 [T4])

[T2] to 2011 [T4])

[T3] to 2011 [T4])

Was there a difference in the frequency of APN Intensivist special initiatives conducted over the four time periods compared of the 3-year study period between 2009 and 2011 ?
Compared groups

Compared groups

Compared groups

Number of APN Intensivists initiatives (continuous) \&

Charges billed by APN

Intensivists during the JMH

SICU stay

\&

Data on APN Intensivist initiatives
One-way ANOVA with

Scheffe's for post-hoc analysis 


\section{CHAPTER IV}

\section{Results}

The primary aim of this retrospective study was to examine the effects of Advanced Practice Nurses (APNs) as intensivists in a surgical intensive care unit (SICU) on patient outcomes, healthcare charges, and APN Intensivist services in the SICU. The study examined the dose effect of APN Intensivists in a surgical intensive care unit (SICU) on patient outcomes, healthcare charges, APN Intensivists service. The study was designed to address the following research questions: (1) Is there a difference in patient outcomes (HAIs [i.e., VAP, CAUTI], pressure ulcers, patient-initiated accidental removal of devices [i.e., self-extubation, removal of venous access, removal of urinary catheters, and removal of feeding tubes], sedation management, post-surgical glycemic control, and ventilator weaning and ventilator days) and SICU length of stay [LOS] when the ICU was staffed by: (a) two APN Intensivists per shift 24/7 (T1) compared to zero to one APN Intensivist per day (T4), (b) one APN Intensivist per shift (T2) compared to zero to one APN Intensivist per day (T4), and (c) one APN Intensivist per shift but not for seven days (T3) compared to zero to one APN Intensivist per day (T4)?, (2) Is there a difference in health care charges for SICU length of stay and APN Intensivists' services comparing T1 versus T4; T2 versus T4; and T3 versus T4 ICU staffing?, and (3) Is there a difference in the frequency of APN Intensivist special initiatives conducted over the four time periods (T1, T2, T3, and T4) between 2009 and 2011? This chapter presents the characteristics of the samples and the results of the data analysis for each research question. Sources of data to address each research question are presented in Table 3. 
Table 3

Study's Sources of Data

Sample

Patients' Demographics

Diagnoses

SICU Length of Stay (LOS)

APN Intensivists’ Demographics

\section{Unit Aggregate}

Healthcare Associated Infections (HAIs)

[VAP, CAUTI]

VAP Rates

Ventilator Days

CAUTI Rates

Catheter Days

Pressure Ulcers

Post-surgical Glycemic Index

Total Charges for APN Intensivists'

Services

APN Intensivists' Initiatives

\section{APN Intensivists, Registered Nurses, and Medical Staffing}

Following IRB approvals from FIU and JMH, data were collected on APN characteristics, unit staffing, patient outcomes, healthcare charges, APN Intensivists services, and APNs special initiatives over the 4 study time periods. 
Table 4

APN Intensivists Demographics

\begin{tabular}{|c|c|c|c|c|}
\hline $\begin{array}{l}\text { Independent } \\
\text { Variables }\end{array}$ & T1 & T2 & T3 & T4 \\
\hline $\begin{array}{l}\text { Number of } \\
\text { APNs }\end{array}$ & 14 & 13 & 10 & 7 \\
\hline Age [M (SD)] & $41.4(8.20)$ & $40.8(8.46)$ & $40.3(8.31)$ & $43(6.65)$ \\
\hline \multicolumn{5}{|l|}{ Race: } \\
\hline W & 6 & 5 & 4 & 2 \\
\hline B & 2 & 2 & 2 & 2 \\
\hline $\mathrm{H}$ & 6 & 6 & 4 & 3 \\
\hline $\begin{array}{l}\% \text { Specialty } \\
\text { Certified }\end{array}$ & $11(78.5 \%)$ & $10(76.9 \%)$ & $7(70 \%)$ & $5(71.4 \%)$ \\
\hline $\begin{array}{l}\text { Years Of } \\
\text { Experience [M } \\
(\mathrm{SD})]\end{array}$ & $3.9(3.83)$ & $4.2(2.93)$ & $4.5(3.02)$ & $6.9(2.54)$ \\
\hline \multicolumn{5}{|l|}{ Gender: } \\
\hline Male & $2(14 \%)$ & $2(15 \%)$ & $2(20 \%)$ & $2(29 \%)$ \\
\hline Female & $12(86 \%)$ & $11(85 \%)$ & $8(80 \%)$ & $5(71 \%)$ \\
\hline
\end{tabular}

Notes: $\mathrm{W}=$ Whites, $\mathrm{B}=$ Blacks, $\mathrm{H}=$ Hispanics

The APN Intensivists group consisted of 14 APNs at T1 then differed in numbers, specialties and certifications from T2 through T4. All APNs were Master's prepared nurse practitioners; one was also a $\mathrm{PhD}$ graduate at $\mathrm{T} 1$ and two were doctoral candidates at the end of T4; over 70\% of all APNs were specialty-certified. At T1 (January 2009 to December 2009) the APNs characteristics were: 14 APNs with the mean age of 41.4 
years $[\mathrm{SD}=8.20]$, ages ranged from 27 to 54 years, 12 were females $(86 \%)$, there were 6 White non-Hispanic, 6 Hispanic, and 2 Black non-Hispanic. Of these 14 APNs, one was a $\mathrm{PhD}$ graduate and 11 were specialty-certified. There were 10 Acute Care nurse practitioners (including one APN dually prepared as an Acute Care nurse practitioner and as a Family) and four Adult Care nurse practitioners. Their mean experience as practitioners was 3.9 years $[\mathrm{SD}=3.83]$ ranging from less than one year of experience to 11 years.

At T2 (January 2010 to June 2010) the APNs characteristics were: 13 APNs with the mean age of 40.8 years [SD: 8.46], ages ranged from 27 to 54 years, 11 were females (85\%), there were 5 White non-Hispanic, 6 Hispanic, and 2 Black non-Hispanic. Of these 13 APNs, one was a PhD graduate and 10 were specialty-certified. There were nine Acute Care nurse practitioners, four Adult Care nurse practitioners, and one dually prepared as a Family and as an Acute Care nurse practitioner. Their mean experience as practitioners was 4.2 years $[\mathrm{SD}=2.93]$ ranging from less than one year of experience to 10 years.

At T3 (July 2010 to December 2010) the APNs characteristics were: 10 APNs with the mean age of 40.3 years [ $\mathrm{SD}=8.31$ ], ages ranged from 30 to 51 years, 8 were females $(80 \%)$, there were 4 White non-Hispanic, 4 Hispanic, and 2 Black non-Hispanic. Of these 10 APNs one was a PhD student and one was an $\mathrm{EdD}$ student and seven were specialty-certified. There were seven Acute Care nurse practitioners and three Adult Care nurse practitioners. Their mean experience as practitioners was 4.5 years $[\mathrm{SD}=$ 3.02] ranging from one year of experience to 10 years. 
At T4 (January 2011 to December 2011) the APNs characteristics were: 7 APNs with the mean age of 43 years [SD=6.65], ages ranged from 33 to 51 years, 5 were females (71\%), there were 2 White non-Hispanic, 3 Hispanic, and 2 Black non-Hispanic. Of these 7 APNs one was a PhD candidate and one was an EdD candidate and 5 were specialty-certified. There were three Acute Care nurse practitioners and four Adult Care nurse practitioners. Their mean experience as practitioners was 6.9 years [ $\mathrm{SD}=2.54]$ ranging from four years of experience to 11 years.

From $\mathrm{T} 1$ to $\mathrm{T} 4$ the number of APNs decreased by $50 \%$. There was no statistically significance difference in the age and race of the APNs however the number of males increased from $14 \%$ at $\mathrm{T} 1$ to $29 \%$ at $\mathrm{T} 4$. The number of certified APNs decreased from $78.5 \%$ at $\mathrm{T} 1$ to $71.4 \%$ at $\mathrm{T} 4$. The mean years of APN experience increased from 3.9 years at $\mathrm{T} 1$ to 6.9 at $\mathrm{T} 4$.

Data on SICU nursing staffing for the four-time periods (T1 through T4) were not made available to the Principal Investigator. Staffing characteristics (age, gender, race, education, certifications, years of experience as nurses, and years of experience in the unit as critical care nurses) for the three years of the study were not able to be abstracted and thus were unable to be reported.

The medical staffing characteristics for the total group over the four-time periods consisted of a team of physicians, led by an attending physician, and including fellows, third-year residents, and interns. Their education and professional level consisted of one surgical attending physician (surgeon) supervising two surgical third-year fellows, one third-year surgical resident, and up to four first-year interns from different medical specialties including anesthesia, surgery, and neurosurgery. The attending physician in 
charge of the unit rotates from daily to up to seven days coverage of the unit. The fellows covered the unit for a period of a month alternating between them with some having 24-hour shifts during their month rotation. The remaining days were covered by the third-year resident who covers the unit from 21:00 until the following day's morning rounds. The first-year interns (PG-1) alternate with one covering night shift, while the other three cover the unit during the day time. The numbers and types of medical staff were consistent throughout the 4 time-periods (T1-T4).

\section{Sample}

From over 1,000 charts of post-operative patients admitted to JMH's SICU that met the study inclusion criteria over the study time periods, 816 were randomly selected (204 per for each T1-T4). This was done by matching inclusion criteria with patients' FIN (financial identifier number). The resulting report was aggregate data except for demographics.

The characteristics for the total patient group over the four time-periods (T1-T4) consisted of 816 patients with the mean age of 60 years [SD=15.29]; ages ranging from 20 to 98 years, 343 females [42\%], 471males [53\%], ethnically composed of 291 [36\%] White non-Hispanic, 239 [29\%] Hispanic, 257 [32\%] Black non-Hispanic and 27 [3\%] of other race or ethnicities. For T3 and T4 one chart in each time period lacked demographic data. 
Table 5

Patient Sample Demographics

\begin{tabular}{|c|c|c|c|c|}
\hline & T1 & $\mathbf{T 2}$ & T3 & T4 \\
\hline $\begin{array}{l}\text { Sample } \\
\text { Size (N) }\end{array}$ & 204 & 204 & 203 & 203 \\
\hline $\begin{array}{l}\text { Age } \\
{[\mathrm{M}(\mathrm{SD})]}\end{array}$ & $60.3(13.51)$ & $61.8(14.65)$ & $59.1(16.17)$ & $58.9(16.86)$ \\
\hline $\begin{array}{l}\text { Gender } \\
n,(\%)\end{array}$ & $n=204$ & $n=204$ & $n=203$ & $n=203$ \\
\hline Males: & $\begin{array}{l}99 \\
(49 \%)\end{array}$ & $\begin{array}{l}132 \\
(64 \%)\end{array}$ & $\begin{array}{l}114 \\
(56 \%)\end{array}$ & $\begin{array}{l}126 \\
(62 \%)\end{array}$ \\
\hline Females: & $\begin{array}{l}105 \\
(51 \%)\end{array}$ & $\begin{array}{l}72 \\
(36 \%)\end{array}$ & $\begin{array}{l}89 \\
(43 \%)\end{array}$ & $\begin{array}{l}77 \\
(38 \%)\end{array}$ \\
\hline \multicolumn{5}{|l|}{$\begin{array}{l}\text { Race } \\
n,(\%)\end{array}$} \\
\hline Blacks & $\begin{array}{l}60 \\
(30 \%)\end{array}$ & $\begin{array}{l}69 \\
(34 \%)\end{array}$ & $\begin{array}{l}81 \\
(40 \%)\end{array}$ & $\begin{array}{l}81 \\
(40 \%)\end{array}$ \\
\hline Whites & $\begin{array}{l}123 \\
(60 \%)\end{array}$ & $\begin{array}{l}39 \\
(19 \%)\end{array}$ & $\begin{array}{l}46 \\
(23 \%)\end{array}$ & $\begin{array}{l}49 \\
(24 \%)\end{array}$ \\
\hline Hispanics & $\begin{array}{l}15 \\
(7 \%)\end{array}$ & $\begin{array}{l}91 \\
(45 \%)\end{array}$ & $\begin{array}{l}71 \\
(35 \%)\end{array}$ & $\begin{array}{l}62 \\
(31 \%)\end{array}$ \\
\hline Other & $\begin{array}{l}6 \\
(3 \%)\end{array}$ & $\begin{array}{l}5 \\
(2 \%)\end{array}$ & $\begin{array}{l}5 \\
(2 \%)\end{array}$ & $\begin{array}{l}11 \\
(5 \%)\end{array}$ \\
\hline
\end{tabular}

The patient sample consisted of surgical patients admitted to the SICU postoperatively. The five most common admitting diagnoses during the study's period were liver transplant, kidney and pancreas transplant, cardiac valve repairs and other major associated cardiothoracic procedures, including coronary bypass with cardiac 
catheterization, and kidney and ureter procedures for neoplasms. The five most common diagnoses in each time period are listed in Table 6.

Table 6

Sample Admitting Diagnoses by Time-Periods

\begin{tabular}{|c|c|c|c|c|c|}
\hline \multicolumn{3}{|c|}{$\underline{\mathbf{T 1}}$} & \multicolumn{3}{|c|}{$\underline{\mathbf{T} 2}$} \\
\hline Diagnosis & $\mathbf{N}$ & $\%$ & Diagnosis & $\mathbf{N}$ & $\%$ \\
\hline $\begin{array}{l}\text { Amputation for } \\
\text { Circulatory } \\
\text { Disease } \\
\text { Coronary }\end{array}$ & 7 & 3.4 & $\begin{array}{l}\text { Cardiac Valve } \\
\text { Repair }\end{array}$ & 14 & 6.8 \\
\hline $\begin{array}{l}\text { Bypass with } \\
\text { Catheterization }\end{array}$ & 5 & 2.4 & Liver Transplant & 10 & 4.9 \\
\hline $\begin{array}{l}\text { Kidney and } \\
\text { Ureter } \\
\text { Procedures }\end{array}$ & 4 & 1.9 & $\begin{array}{l}\text { Kidney and } \\
\text { Pancreas } \\
\text { Transplant }\end{array}$ & 7 & 3.4 \\
\hline $\begin{array}{l}\text { Cardiac Valve } \\
\text { Repair }\end{array}$ & 4 & 1.9 & $\begin{array}{l}\text { Coronary Bypass } \\
\text { with } \\
\text { Catheterization }\end{array}$ & 6 & 2.9 \\
\hline $\begin{array}{l}\text { Kidney and } \\
\text { Pancreas } \\
\text { Transplant }\end{array}$ & 4 & 1.9 & $\begin{array}{l}\text { Amputation for } \\
\text { Musculoskeletal } \\
\text { and Connective } \\
\text { Tissue }\end{array}$ & 5 & 2.4 \\
\hline & $\underline{\Gamma 3}$ & & \multicolumn{3}{|c|}{$\underline{\mathrm{T} 4}$} \\
\hline Diagnosis & $\mathbf{N}$ & $\%$ & Diagnosis & $\mathbf{N}$ & $\%$ \\
\hline $\begin{array}{l}\text { Liver } \\
\text { Transplant } \\
\text { Kidney and }\end{array}$ & 9 & 4.4 & Cholecystectomy & 7 & 3.4 \\
\hline $\begin{array}{l}\text { Pancreas } \\
\text { Transplant }\end{array}$ & 8 & 3.9 & Liver Transplant & 6 & 2.9 \\
\hline $\begin{array}{l}\text { Other } \\
\text { Digestive } \\
\text { System } \\
\text { Surgical }\end{array}$ & 4 & 1.9 & $\begin{array}{l}\text { Kidney and } \\
\text { Pancreas } \\
\text { Transplant }\end{array}$ & 5 & 2.4 \\
\hline $\begin{array}{l}\text { Other Vascular } \\
\text { Procedures }\end{array}$ & 4 & 1.9 & $\begin{array}{l}\text { Major chest } \\
\text { Procedures }\end{array}$ & 4 & 1.9 \\
\hline $\begin{array}{l}\text { Post-operative } \\
\text { or Post- } \\
\text { Traumatic }\end{array}$ & 4 & 1.9 & $\begin{array}{l}\text { Kidney and } \\
\text { Ureter } \\
\text { Procedures for } \\
\text { Neoplasms }\end{array}$ & 4 & 1.9 \\
\hline
\end{tabular}




\section{Differences in Patient Outcomes Comparing T1 versus T4; T2 versus T4; and T3 versus T4 ICU staffing}

The sample to address this and the following study question consisted of 816 charts of post-operative patients admitted to the JMH's SICU between the years 2009 through 2011. For 2009 through 2011 (T1, T2, T3, and T4), a random sample of 204 patient charts in each time-period (total $\mathrm{N}=816$ ) that met study criteria were selected for review. Each of the resulting four groups of charts represented a different level of APN Intensivist staffing.

The original plan was to conduct chart abstraction from each of the randomly selected charts to examine the HAIs (VAP, CAUTI), pressure ulcers, patient-initiated accidental removal of devices, sedation management, post-surgical glycemic control, ventilator weaning and ventilator days, and SICU length of stay outcomes. Acute Physiology and Chronic Health Evaluation-II (APACHE-II) scores were to be used to control for level of patient acuity. Review of data for 816 charts became problematic during the period of data collection due to changes in the health system's methods of documentation and data collection for key outcomes, major changes in the system and in its leadership, and several changes in key personnel providing access to the study data.

Data on patient-initiated accidental removal of devices, sedation management, and ventilator weaning, ventilator days, and APACHE-II scores were not consistently documented in the charts and thus provided unreliable data or the data were not available in the chart data. Data on pressure ulcers were reported quarterly and were obtained from the National Data of Quality Indicators (NDQI) JMH coordinator. Data on post-surgical glycemic control were obtained from a database managed by the APN Intensivists. Data 
on SICU length of stay (LOS) were obtained from JMH Information Technology Department.

Data on HAIs (VAP, CAUTI) were obtained from the JMH Infection Control Prevention (IPC) and from the Vigilanz ${ }^{\circledR}$ database (an internal control database that tracks HAIs). For the measures of HAIs (VAP, CAUTI) data were abstracted from aggregate data reports linking these measures to Diagnostic Related Groups (DRGs) reported by JMH Information Technology Department and facilitated by the Quality and Patient Safety Office. These data were obtained by combining data from the Vigilanz ${ }^{\circledR}$ database (JMH internal control for HAIs) and data from the JMH Infection Control Prevention database.

Pressure Ulcer measures data were obtained by abstraction of the JMH's NDQI report submitted quarterly for the years included in the study. Post-surgical glycemic control measures data were obtained from internal SICU database initiated and maintained by SICU APNs Intensivists. SICU Length of Stay (LOS) data were abstracted from the Revenue Compass ${ }^{\circledR}$ Financial database facilitated by the Quality and Patient Safety Office linking the charges in SICU by patient Financial Identification Number (FIN) to SICU stay. 


\section{Healthcare-Associated Infections (HAIs)}

Table 7

SICU Healthcare-Associated Infections (HAIs)

T1

4.51 days

LOS

Ventilator Days

Infections

(VAP)

\section{Average SICU}

LOS
Ventilator Days
Infections

Urinary

Catheter Days

Infections

(CAUTI)

CAUTI Rate
2.57 per 1000

3.40 per 1000

Ventilator Days

3193

3342

2868

6882

0.82 per $1000 \quad 0.68$ per 1000

Ventilator Days Ventilator Days Ventilator Days
8

2.39 per 1000

2.19 per 1000

Catheter Days
Catheter Days
1.17 per 1000

1.60 per 1000

Catheter Days Catheter Days

The mean number of ventilator days for the study was 2,226.5 days, ranging from 1,219 days in $\mathrm{T} 3$ to 4,367 days in T4. The number of cases of ventilator associated pneumonia (VAP) ranged from one in T3 to six cases in T2. The VAP rates, calculated by dividing the number of infections (i.e., VAP) by the number of ventilator days in the 
month (i.e., number of intubated patients multiplied by days of the month) and multiplying that result by 1000 for every time period, ranged from 0.68 at T4 to 3.40 at T2. For catheter days the range was from 2,862 catheter days for T3 to 3,342 catheter days for T2. The number of catheter-associated urinary tract infections (CAUTI) ranged from four cases in $\mathrm{T} 3$ to 11 cases in T4. The CAUTI rates calculated by dividing the number of infections (i.e., CAUTI) by the number of urinary catheter days (i.e., number of catheterized patients multiplied by days of the month) and multiplying that result by 1000 for every time period, ranged from 1.17 at $\mathrm{T} 3$ to 2.39 at $\mathrm{T} 2$.

When comparing reported LOS at T1 versus T4, the LOS was greater $(+3.19$ days) at T4 (7.70 days) than on T1 (4.51 days). Similarly, ventilator days (1,557 versus 4,367), catheter days (3,193 versus. 6,802), and infections (i.e., CAUTI) (7 versus 11) were greater at $\mathrm{T} 4$. When comparing reported LOS at $\mathrm{T} 2$ versus $\mathrm{T} 4$ the LOS was greater (+3.77 days) at T4 (7.70 days) than on T2 (3.93 days). Similarly, ventilator days (1,763 versus 4,367$)$, catheter days $(3,342$ versus. 6,802$)$, and infections (i.e., CAUTI) (8 versus. 11) were greater at $\mathrm{T} 4$. When comparing reported LOS at $\mathrm{T} 3$ versus $\mathrm{T} 4$ the LOS was greater ( +0.54 days) at T4 (7.70 days) than on T2 (7.16 days). Similarly, infections (i.e., VAP [1 versus. 3] and CAUTI [4 versus 11]) and CAUTI rate (1.17 per 1000 catheter days versus. 1.60 per 1000 catheter days) were greater at $\mathrm{T} 4$.

In summary, reported LOS, ventilator days, catheter days and CAUTI increased at $\mathrm{T} 4$ compared to $\mathrm{T} 1, \mathrm{~T} 2$, and $\mathrm{T} 3$. VAP infection was decreased in $\mathrm{T} 4$ (3 cases) when compared to T1 (4 cases) and T2 (6 cases), but not with T3 (1 case). VAP rates were decreased in T4 (0.68 per 1000 ventilator days) when compared to T1 (2.57 per 1000 ventilator days), T2 (3.40 per 1000 ventilator days), and T3 (0.82 per 1000 ventilator 
days). Furthermore, CAUTI rates were decreased in T4 (1.60 per 1000 catheter days) when compared to T1 (2.19 per 1000 catheter days), T2 (2.39 per 1000 catheter days), but increased when compared with T3 (1.17 per 1000 catheter days). It is important to note when interpreting these results that there is a time span difference across time periods. T1 and T4 are composed of 12 calendar months (i.e., 2009 and 2011 respectively) versus T3 and T4 which are both 6-month periods for 2010 (i.e., January through June and July through December 2010 respectively). Because data generated for this analysis were aggregate data it was not possible to divide $\mathrm{T} 1$ and $\mathrm{T} 4$ into 6-month periods to facilitate comparing the first six months of each study year.

\section{Pressure Ulcers}

Table 8

Incidence of Pressure Ulcers in the SCIU by Time-Periods (Quarters)

\begin{tabular}{lllllllllllll}
\hline & T1a & T1b & T1c & T1d & T2a & T2b & T3c & T3d & T4a & T4b & T4c & T4d \\
\% & 22.2 & 5.9 & 14.3 & 19.1 & 3.7 & 26.5 & 20 & 17.1 & 20.6 & 15.1 & 24.2 & 7.4 \\
& & & & & & & & & & & & \\
\hline
\end{tabular}

Note. Italic letters $=$ first $(a)$, second $(b)$, third $(c)$, and fourth $(d)$ quarters of each T

Pressure ulcers data were abstracted by years and then reported as quarters to follow the reporting system to NDQI used by JMH. Each quarter was labeled $a$ through $d$ to reflect the corresponding quarter for each time period. It was not possible to abstract, analyze and report sample means and standard deviation for the SICU sample because the aggregate data did not show how many patients were sampled for the report. 
Means for each time period; and then comparison of the first two quarters and last quarters of each $\mathrm{T}$ was not possible.

Pressure ulcers data reflects the incidence (total number of patients with pressure ulcers in the time period divided by the total number of patients for the time period) of unit acquired pressure ulcers of stage II and above and the pressure ulcer prevention measures implemented in SICU by APNs Intensivists.

Table 9

Compliance of Pressure Ulcers Prevention Measures in the SICU

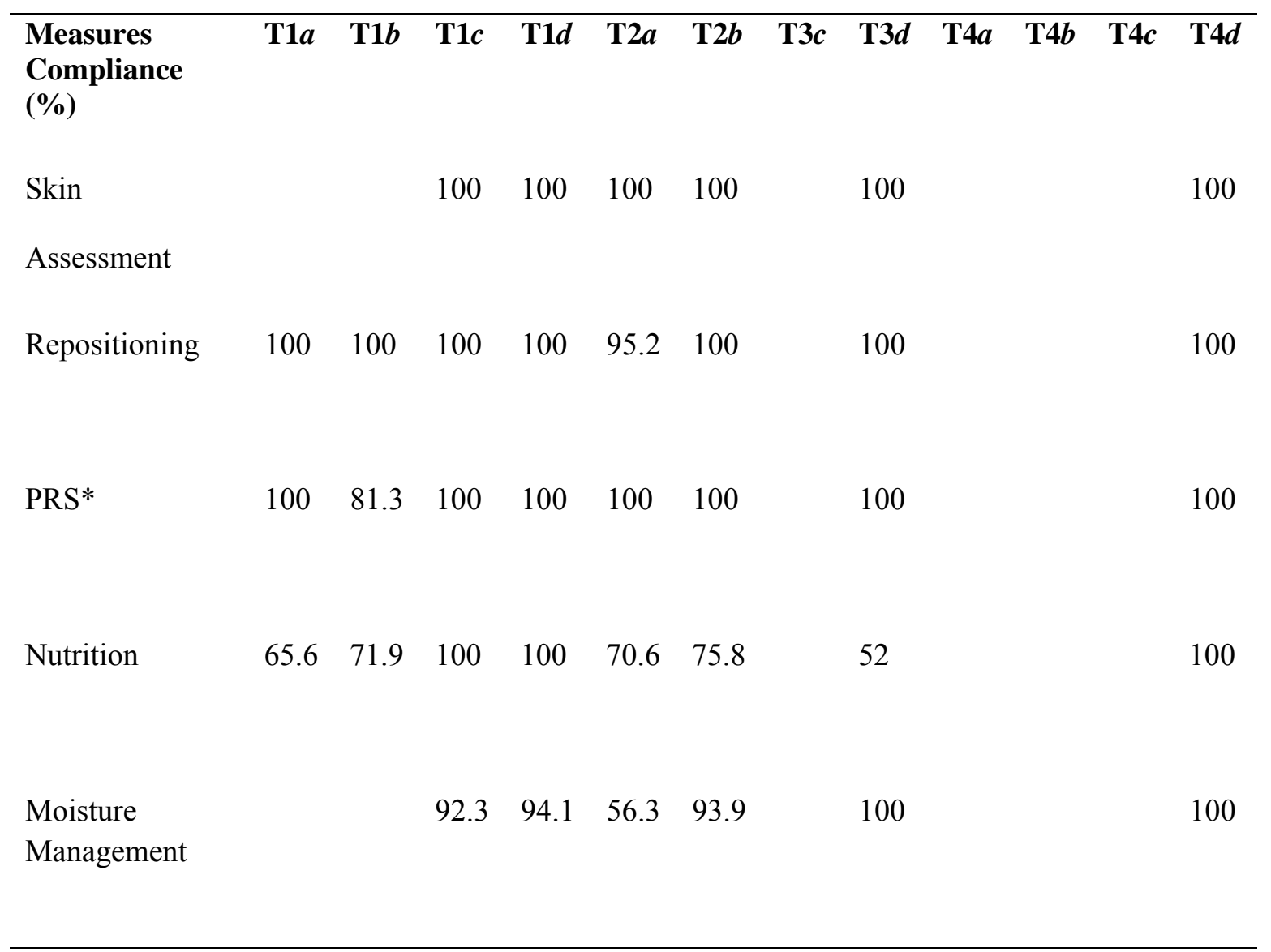

Note. $P R S^{*}=$ Pressure Relief Surface; Italic letters $=$ first $(a)$, second $(b)$, third $(c)$, and fourth $(d)$ quarters of each $\mathrm{T}$. 
For T1 (2009 first quarter) and T1 (2009 second quarter) data for number of patients surveyed, skin assessment and moisture management were missing. Data for T3 (2010 third quarter) and for the first three quarters of 2011 (i.e., T4a, T4b, and T4c respectively) on prevention measures were missing. Skin assessments for the reported time frames were completed $100 \%$. Repositioning of patients was attained also at a $100 \%$ rate for the reported time frames except for T2 (2010 first quarter) that was reported at 95.2\%. Pressure relief surface (PRS) intervention was reported for T1 (2009 second quarter) at an $81.3 \%$ rate but $100 \%$ compliance was reported for the rest of the reported time frames. Nutrition compliance was reported as high as a $100 \%$ rate for T1 (2009 third quarter), T1 (2009 fourth quarter), and T4 (2011 fourth quarter) and as low as $65.6 \%$ for T1 (2009 first quarter), $71.9 \%$ for T1 (2009 second quarter), $70.6 \%$ for T2 (2010 first quarter), and 75.8\% for T2 (2010 second quarter). The lowest reported value of all reported time frames was 52\% for T3 (2010 fourth quarter). Similarly, for the moisture management compliance measure a hundred percent was reported for T3 (2010 fourth quarter) and T4 (2011 fourth quarter) but lower values were reported for T1 (2009 third quarter [92.3\%]), T1 (2009 fourth quarter [94.1\%]), T2 (2010 first quarter [56.3\%]), and T2 (2010 second quarter [93.9\%]) respectively.

When comparing the incidence of pressure ulcers in the first two quarters of T1 $(28.1 \%)$ versus the first two quarters of T4 $(35.7 \%)$ incidence was increased $(+7.6)$ in T4. When comparing the incidence of pressure ulcers in the first two quarters of T2 $(30.2 \%)$ versus the first two quarters of T4 (35.7\%) incidence was increased $(+5.5)$ in T4. Data were not available to compare the incidence of pressure ulcers in the first two quarters of $\mathrm{T} 3$ versus the first two quarters of T4. When comparing the incidence of pressure ulcers 
in the second two quarters of T1 $(33.4 \%)$ versus the second two quarters of T4 $(31.6 \%)$ incidence was decreased (-1.8) in T4. Data were unavailable to compare the incidence of pressure ulcers in the second quarters of $\mathrm{T} 2$ versus the second quarter of $\mathrm{T} 4$. When comparing the incidence of pressure ulcers in the second two quarters of T3 $(37.1 \%)$ versus the second two quarters of T4 (31.6\%) incidence was decreased $(-5.5)$ in T4.

Comparing study time points on pressure ulcer prevention measures including skin assessment measures, repositioning of patients, use of pressure relief surfaces, nutrition assessment, and moisture management comparisons implemented in the SICU by APN Intensivists was not possible because of missing data. However, the largest amount of missing data was in the first three quarters of T4 when the number of APNs was the lowest.

\section{Post-Surgical Glycemic Control}

Table 10

Unit Glucose Control Maintenance

\begin{tabular}{cccc}
\hline & T2 & T3 & T4 \\
& 13 APNs & 10 APNs & 7 APNs \\
Total Admissions & 43 & 139 & 263 \\
Fallouts (Glucose $>$ & 2 & 13 & \\
$200 \mathrm{mg} / \mathrm{dl})$ & & & \\
& & & \\
\hline
\end{tabular}


Post-surgical glycemic control measures were collected since the inception of this APN Intensivists' initiative from May 2010 to December 2011. Data for T1 were not available and for T2 were available for only two months (May and June, 2010). Data were complete and available for $\mathrm{T} 3$ and $\mathrm{T} 4$. Of the 43 cardiothoracic patients admitted to SICU on T2 only 2 (4.7\%) fallouts (i.e., glucose values over $200 \mathrm{mg} / \mathrm{dl}$ ) for the measure were recorded. For T3 only 13 (9.4\%) fallouts of 139 admissions were recorded. For T4 only 18 (6.8\%) fallouts of 263 admissions were recorded. Data for the month of November 2011 were adjusted and only 15 out of 18 were considered for the study. For that particular month glucose readings for 2 out of 18 admissions were documented only for Day 1 and the others were never entered for days 1 and 2 respectively so they were excluded from the data.

Data on $\mathrm{T} 1$ were not available for a patient outcome comparison between $\mathrm{T} 1 \mathrm{vs}$. T4. When comparing data on T2 (two months reported) versus T4 the percentage of measure fallouts was lower for T2 (4.7\%) than for T4 (6.8\%). When comparing T3 versus $\mathrm{T} 4$ the percentage of fallouts was greater in $\mathrm{T} 3(9.4 \%)$ than of $\mathrm{T} 4(6.8 \%)$ however, an equal variances $t$ test failed to reveal a statistically significant difference between the mean number of glucose fallouts (i.e., glucose levels greater than $200 \mathrm{mg} / \mathrm{dl})(\mathrm{M}=161.67$, $\mathrm{SD}=34.14)$ and $\mathrm{T} 4(\mathrm{M}=142.84, \mathrm{SD}=40.00), t(223)=1.40, p=.17$. 
Table 11

Unit Glucose Fallouts Comparison Between Time Periods

Glucose Fallouts Means

T3 T4

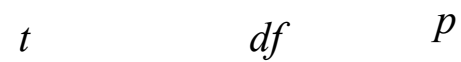

T3

161.67

142.84

1.40

223

.17

$(40.00)$

Note. ${ }^{*}=p \leq .05$, Standard deviations appear in parentheses below means.

\section{Length of Stay (LOS)}

Table 12

SICU Length of Stay by Time Periods

\begin{tabular}{lllll}
\hline & T1 & T2 & T3 & T4 \\
N & 204 & 204 & 204 & 204 \\
Mean & 4.51 & 3.93 & 7.16 & 7.70 \\
SD & 5.883 & 4.092 & 13.028 & 15.024 \\
Range & $0 *-50$ & $0 *-31$ & $0 *-78$ & $0 *-97$ \\
\hline
\end{tabular}

Note. $*=$ less than 24 hours 
The length of stay (LOS) for patients in the SICU varied across time periods. The length of stay sampled for January through December 2009 (T1) ranged from less than a day ( 24 hours) to 50 days with a mean stay of 4.51 days $(\mathrm{SD}=5.88)$. For $\mathrm{T} 2$ the mean LOS was of 3.93 days $(\mathrm{SD}=4.09)$ and a range of less than a day ( 24 hours) to 31 days. For T3 the mean LOS was of 7.16 days $(\mathrm{SD}=13.0)$ and a range of less than a day $(24$ hours) to 78 days. The mean LOS for the last time-period of the study (T4) was 7.70 days $(\mathrm{SD}=15.4)$ and a range of less than a day (24 hours) to 97 days.

When comparing T1 LOS vs. T4 LOS a t-test failed to reveal a statistically significant difference between the mean numbers of days (LOS) in SICU at T1 $(\mathrm{M}=4.31$, $S D=5.01)$, and that of $\mathrm{T} 4(\mathrm{M}=8.18, \mathrm{SD}=14.31), t(10.14)=.90, \mathrm{p}=.39$. When comparing T2 LOS vs. T4 LOS a t-test failed to reveal a statistically significant difference between the mean numbers of days (LOS) in SICU at T2 $(\mathrm{M}=3.98, \mathrm{SD}=$ 4.17), and that of $\mathrm{T} 4(\mathrm{M}=2.91, \mathrm{SD}=2.01), t(202)=0.85, p=.40$. When comparing $\mathrm{T} 3$ LOS vs. T4 LOS a t-test failed to reveal a statistically significant difference between the mean numbers of days (LOS) in SICU at T3 $(\mathrm{M}=7.38, \mathrm{SD}=13.34)$, and that of T4 $(\mathrm{M}=$ $3.27, \mathrm{SD}=3.32), t(202)=1.02, p=.31$. There was no statistically significant difference in the LOS among all compared time-periods. 
Table 13

Length of Stay Comparison Between Time Periods

\section{Length of Stay Means}

T1

T1

T4

$T$

$d f$

$p$

4.31

8.18

.90

10.14

.39

T2

$\mathrm{T} 2$

T4

$T$

$d f$

$p$

.85

202

.40

$\mathrm{T} 3$

T3

$\mathrm{T} 4$

$T$

$d f$

$p$

7.38

1.02

202

.31

Note. ${ }^{*}=p \leq .05$, Standard deviations appear in parentheses below means.

Differences in Healthcare Charges for SICU Length of Stay and APN Intensivists'

Services Comparing T1 vs. T4; T2 vs. T4; and T3 vs. T4 ICU Staffing

For each of the four study periods (T1, T2, T3, and T4), total healthcare charges for SICU patient stays for the sample were not available. These data could have been abstracted from the Revenue Compass ${ }^{\circledR}$ Financial database but access to the database was restricted by JMH administration thus preventing its report. Total charges for APN 
Intensivists' services were abstracted from a report generated in December 2012 by the McKesson ${ }^{\circledR}$ financial database at JMH. This report was submitted to the JMH Financial Department and was made available to the Principal Investigator.

\section{Charges for APN Intensivists' Services}

The charges for APN Intensivists' services were obtained from the financial report generated by the JMH Financial Office on SICU ARNPs' charges. These amounts reflected charges attributed to SICU APN Intensivists from the inception of billing for services in the SICU (i.e. 2009) to the date of the report generated on December 7, 2012. The report tallied the number of units (procedures) paired with corresponding charges for each billing period. It was not possible to obtain data on APN charges for procedures for individual patients.

For each of the four study periods (T1, T2, T3, and T4), total healthcare charges for APN Intensivists' services for the sample were \$90,478. For T1 (January 2009 to December 2009) charges for APN Intensivists' services over this 12 month period were $\$ 11,268$. This sum reflects only the months of September, October, November, and December for that time period; data were missing for January through August.

For T2, (January 2010 to June 2010) charges for APN Intensivists' services over this 6 month period were $\$ 18,691$. For T3 (July 2010 to December 2010) charges for APN Intensivists' services over this 6 month period were $\$ 8,792$. This sum excludes missing data for the month of August 2010. At T4 (January 2011 to December 2011) charges for APN Intensivists' services over this 12 month period were $\$ 51,727$. This sum reflects the total charges for T4 except for missing data for December 2011. 
Charges for APN services increased over the 4 time periods from $\$ 11,268$ at T1 to $\$ 51,727$ at $\mathrm{T} 4$.

The increased charges for APN services could be attributed to a change in the system for capturing charges for APN services. Prior to 2012 billing for APN services was inconsistent and was done manually. In 2012 a system was put in place to capture APN billing electronically. Individual charges for procedures performed by APNs were unavailable for abstraction from financial data. However, a list of the APNs' performed procedures was available for abstraction for each time-period. These listed procedures reflected the procedures submitted for billing as service charges from APNs' during each of study's time-periods.

For $\mathrm{T} 1$ the procedures listed were insertions of central venous lines, arterial catheters, and orogastric and nasogastric tubes with stents, reposition of gastric tubes, and venipuncture. For T2, the procedures included those in $\mathrm{T} 1$ in addition to insertion of peripherally inserted central catheters (PICC), reposition and replacement of PICC lines, bronchoscopy, and ultrasound guided vascular access. There were no additional procedures listed for $\mathrm{T} 3$ other than the ones described in T2. For T4, APNs' charges for services included those in $\mathrm{T} 3$ and percutaneous tracheostomies, and reposition or replacement of central venous lines and arterial catheters. One can assume then that charges for APNs services increased over the 4 time periods, (from $\$ 11,268$ at T1 to $\$ 51,727$ at T4), due to the addition of new procedures to the services provided by APNs or to the frequency in number of these procedures performed by APNs for each timeperiod. 


\section{Differences in the Frequency of APN Intensivist Special Initiatives Conducted Over the Four Time Periods (T1, T2, T3, and T4)}

For each of the four study time-periods, (T1, T2, T3, T4) data were collected on major APN Intensivists' initiatives including new protocols, development of databases for quality outcomes and safety patient indicators, etc. Data for these initiatives were collected from stored databases available only to APN Intensivists on a separate computer drive in the JMH SICU menu (i.e., V-Drive). These data were deidentified.

The following table depicts the four major APN Intensivists initiatives and their duration from implementation to end through the four-time periods (T1-T4) of the study.

Table 14

APN Intensivists Initiatives Over Four Time-Periods (T1-T4)

$\begin{array}{llll}\text { T1 } & \text { T2 } & \text { T3 } & \text { T4 }\end{array}$

\begin{tabular}{|c|c|c|c|c|}
\hline $\begin{array}{l}\text { Blood glucose } \\
\text { levels indicator }\end{array}$ & 0 & $\sqrt{ }$ & $\sqrt{ }$ & $\sqrt{ }$ \\
\hline $\begin{array}{l}\text { SCIP initiative } \\
\text { post-operative } \\
\text { day one }\end{array}$ & 0 & $\sqrt{ }$ & $\sqrt{ }$ & 0 \\
\hline $\begin{array}{l}\text { Cardiac } \\
\text { admission } \\
\text { quality checklist }\end{array}$ & 0 & $\sqrt{ }$ & $\sqrt{ }$ & 0 \\
\hline $\begin{array}{l}\text { Daily quality } \\
\text { initiative list }\end{array}$ & 0 & 0 & 0 & $\sqrt{ }$ \\
\hline
\end{tabular}


In T1 (January 2009 through December 2009), when there were 14 APNs, the APNs established themselves as a team. No initiatives were begun during this period. The team began working on identifying the measures that would be assessed and targeted as initiatives. After numerous meetings with nurses, physicians, the infection control team and unit management the selection of measures were decided. Databases were assembled for data collection and short instructional PowerPoint presentations were developed for nursing staff on the selected measures.

In T2 (January 2010 through June 2010) there were 13 APNs and the following three initiatives were established: blood glucose levels indicator, SCIP initiative postoperative day one, and daily quality initiative list. In T3 (July 2010 through December 2010) with 10 APNs there were three initiatives ongoing but no new initiatives had begun. In T4 (January 2011 through December 2011) blood glucose monitoring was continued from T2 and only one new initiative, the cardiac admission checklist, was begun.

The difference in the frequency of APN Intensivists special initiatives conducted over the four time-periods varied in number and types of initiatives followed by these APNs. Blood glucose monitoring was a measure followed from inception at T2 through T4 inclusive. In T4, the cardiac admission checklist was incorporated resulting in two different measures followed for that specific time period (i.e., T4). As for the SCIP initiative post-op day one and daily quality initiative list measures begun during T2, monitoring continued throughout $\mathrm{T} 3$ only. Furthermore, $\mathrm{T} 2$ and $\mathrm{T} 3$ were the only two time-periods with the highest number of initiatives (i.e., 3) consistently being followed by APNs. The number of initiatives followed by APNs later declined in T4 to two but only 
one of the measures begun at T2 (i.e., blood glucose monitoring) continued to be followed. The four major initiatives established by the APN Intensivists group and their development throughout the study time-periods are discussed in detail in the following paragraphs.

Blood Glucose Levels Indicator. For the SICU project the post-surgical glycemic levels indicator was selected as the first core measure to be implemented and monitored by APN Intensivists. Starting in March of 2010 (within T2) the goal was to implement the core measure and attain 100 percent compliance with glucose levels of less than $200 \mathrm{mg} / \mathrm{dl}$ in post-operative surgical cardiothoracic patients admitted to SICU on days one and two postoperatively. A pilot of the initiative was launched for the months of March and April 2010 (T2) and the results were 100 percent attainment of the goal for all admitted postoperative surgical cardiothoracic patients in the SICU. This initiative was then formally incorporated as part of the routine surveillance for APN Intensivists overseeing the cardiothoracic patients in the SICU. This was one of the first initiatives formally incorporated into the daily routine surveillance of APN Intensivists in the unit. Currently, this measure is still followed by the APN Intensivists on the surgical cardiothoracic patients in the SICU.

SCIP Initiative Post-operative Day One. Following the postoperative blood glucose levels indicator other core surveillance measures were implemented by the APN Intensivists in the SICU. Deep Vein Thrombosis (DVT) prophylaxis, perioperative antibiotic compliance, and removal of urinary catheters 48 hours following surgery were the next core measures followed by the APN Intensivists. Starting in April 2010 (T2) throughout August 2010 (T3) these measures were closely followed and monitored by the 
APN Intensivists in the SICU. A database was established to track all SICU admissions and correlate them with these core measures. This database identified whether DVT prophylaxis was ordered and if not the reasons for non-compliance. The database also included compliance with antibiotic therapy as per JMH's perioperative protocol, appropriateness of therapy duration and noncompliance reasons. Lastly, the database identified the need for urinary catheters postoperatively and whether the catheters were removed as per established core measure criteria within 48 hours of placement post operatively.

Cardiac Admission Quality Checklist. Starting in 2010, the APN Intensivists group along with nurses, administrators, and physicians in the SICU partnered to develop and implement a unit-based Surgical Care Improvement Project (SCIP) complying with the Joint Commission on Accreditation of Healthcare Organizations $\left(\mathrm{JCAHO}^{\circledR}\right.$ ) National Hospital Inpatient Quality Measures (JCAHO, 2012). The Joint Commission on Accreditation of Healthcare Organizations $\left(\mathrm{JCAHO}^{\circledR}\right)$ added as core measures in 2003 Surgical Infection Prevention (SIP) measures. Hospitals began collecting core measure data for SIP with patient discharges the following year (2004). The SIP set subsequently transitioned to the Surgical Care Improvement Project (SCIP) measures starting July 1, 2006. The Surgical Care Improvement Project (SCIP) is a national quality partnership of organizations interested in improving surgical care by significantly reducing surgical complications (JCAHO).

SCIP partners include the Steering Committee of 10 national organizations including the American Hospital Association (AHA), Centers for Disease Control and Prevention (CDC), Institute for Healthcare Improvement (IHI), and Joint Commission on 
Accreditation of Healthcare Organizations (JCAHO) who have pledged their commitment and full support for SCIP (JCAHO, 2012). In addition, each of the SCIP target areas is advised by a technical expert panel. These groups have provided technical expertise to ensure the SCIP measures are fully supported by evidence-based research (JCAHO). Although participation is voluntary many of the measures are components of measures being collected by other initiatives, including the Hospital Quality Alliance (HQA), JCAHO core measures, and CMS's 8th Scope of Work, American College of Surgeons initiative (ASC/NSQIP), and IHI's 5 million lives campaign. In summary, SCIP on a national level is a project directed to quality and aimed to improve surgical care by significantly reducing surgical complications. The focus is on quality measures and patient outcomes (JCAHO, 2012).

Daily Quality Initiatives List. The Daily Quality Initiatives (DQI) List (See Appendix 1) was developed to capture the different activities performed by APN Intensivists during their 12-hour shifts in addition to their primary role of clinical patient management and performance of bedside procedures. The list detailed the monitoring of blood glucose levels on post-operative patients to address the blood glucose levels quality measure, the data collection on the SCIP spreadsheet addressing venous thromboembolic (VTE) prophylaxis within 24 hours of admission postoperatively, surgical prophylaxis antibiotics indication and use, and urinary catheter removal within 48-hours postoperatively, APACHE scores calculated for admissions to SICU within the first 24 hours of postoperative admission, indicators for HAIs (i.e., VAP, CLABSI, and CAUTI) and Pressure Ulcer prophylaxis, falls prevention, direct hand hygiene observations on healthcare staff, and the inclusion of miscellaneous activities performed by APN 
Intensivists while on duty. These miscellaneous activities included the participation of APN Intensivists in daily meetings with other members of the interdisciplinary team as in discharge planning meetings, family conferences, and interdisciplinary review meetings (IDRs).

In addition, the list captures the surveillance role of APN Intensivists in monitoring infection rates, incidence and prevalence, and antibiotic stewardship in collaboration with pharmacists and the hospital's Infection Control team. The role of educators is evident throughout the document as it captures the involvement of practitioners in educating staff, patients, and families on the various initiatives addressed in the DQI list.

The APNs in the SICU spearheaded the initiative of monitoring and documenting these quality indicators that addressed IHI's bundles for Ventilator Associated Pneumonia (VAP), Central Line Associated Bloodstream Infection (CLABSI), and Catheter Associated Urinary Tract Infection (CAUTI), Pressure Ulcers (PU) prophylaxis, falls prevention, and hand hygiene observation. These quality indicators were collected and documented in a database on a daily basis by APNs to monitor and ensure compliance with bundle measures and other initiatives. In addition, based on data collected, staff education needs were accessed and a series of short PowerPoint ${ }^{\circledR}$ presentations were developed to be used as teaching tools for staff for each one of the quality indicators measured.

The VAP bundle consisted of measuring whether head of bed was elevated 30 degrees, sedation was interrupted to assess responsiveness, DVT and peptic ulcer prophylaxis was implemented, oral care, and assessment of readiness to extubate. The 
CLABSI bundle consisted of measuring whether line dressing was intact and properly applied, if Biopatch ${ }^{\circledR}$ was used, the catheter site and duration of catheter insertion. The CAUTI bundle consisted of catheter days and whether the catheter was changed or not during the SICU stay.

As for Pressure Ulcers, the location, stage, and etiology of the ulcer were measured. Lastly, the falls initiative consisted of identifying whether the patient was at risk for falls and if the patient was appropriately identified by an identification band specifically for falls precautions. These measures were collected, documented, and entered into the APN Intensivists' database from May 2010 (T2) through August 2010 (T3). The education modules developed to address each one of these measures are still used to train and educate staff by APN Intensivists although the collection of data on these measures is not been currently implemented.

Responding to the need for identifying and addressing the incidence and prevalence of VAP, CAUTI, pressure ulcers, and other patient outcomes, the APN Intensivists' group developed a set of measures in the SICU aimed to address such need. These measures consisted first in creating a database that will capture the adherence to evidence-based practices and standardized guidelines for each particular entity (e.g. VAP, CAUTI, and Pressure Ulcers). Subsequently, educational material in the form of Microsoft ${ }^{\circledR}$ PowerPoint ${ }^{\circledR}$ presentations were developed to educate staff and disseminate knowledge among nursing primarily and ancillary and other healthcare staff involved inpatient care secondarily, on the need to adhere to evidence-based practices and standardized guidelines for each particular entity. Ultimately, protocols were developed and unit-wide implementation of established recommendations and guidelines followed 
with APNs Intensivists responsible for implementing and overseeing the process and providing continuous feedback resulting from the surveillance efforts that the implementation of those protocols generated.

For T1 (January through December 2009), APN Intensivists special initiatives consisted largely in establishing target outcomes and developing the database needed to track the incidence and prevalence of the targeted patient outcomes and adherence to implementation of the adopted guidelines and protocols specific to each outcome. Three tools were developed to measure adherence to protocols set forth by the SCCM's Sepsis Campaign and IHI's recommendations specifically for HAIs (i.e., VAP, CLABSI, and CAUTI) bundles, and two additional ones to capture additional quality indicators like the bundles for falls and pressure ulcers. The resulting five tools were combined in a Microsoft ${ }^{\circledR}$ Excel $^{\circledR}$ document and this was the blueprint for the development of the intended database.

The VAP bundle consists of four elements of care supported by solid level I trials. These four elements are: elevation of the head-of-the-bed to between 30 and 45 degrees, daily sedative interruption or "sedation vacation", and daily assessment of readiness to extubate, peptic ulcer disease prophylaxis, and deep venous thrombosis prophylaxis (unless contraindicated) (IHI, 2010). Other interventions like oral care and subglottic suctioning of secretions were also included in the bundle.

For CAUTI the tool was designed to measure indicators as suggested by the Association for Professionals in Infection Control and Epidemiology (APIC) in 2008. This list of measures included: the use of indwelling bladder catheters only when medically necessary, use of aseptic insertion technique with appropriate hand hygiene 
and gloves, maintaining a sterile closed drainage system, maintaining good hygiene at the catheter-urethral interface, removing catheters when no longer needed, documenting indication for urinary catheter on each day of use, and the use of reminder systems to target opportunities to remove catheters.

The selected measures for Pressure Ulcers in the ICU followed the recommendations issued by the European Pressure Ulcer Advisory Panel (EPUAP) and the Agency for Health Care Policy and Research (AHCPR). The measures used were pressure reducing devices including special mattresses, beds, or cushions, nursing interventions like repositioning every two hours, increased mobility and early ambulation, skin inspection (use of the Braden score), assessment of nutritional and fluid deficits, and patient avoidance of sheer and friction.

As for CLABSI measures like full barrier precautions (i.e., the use of full-sized sterile drapes on the patients and the use of a mask, cap, sterile gown and gloves on the practitioner performing the insertion [Goede \& Coopersmith, 2009]), the anatomic site of insertion, (Subclavian vein access versus femoral), catheter maintenance practices like the use of sustained-release chlorhexidine dressings (i.e., Biopatch ${ }^{\circledR}$ ), daily review of line necessity and the prompt removal of unnecessary central venous catheters (CVCs) (IHI, 2010) were tallied in the developed tool. They included five evidence-based procedures recommended by $\mathrm{CDC}$ (hand hygiene, using full barrier precautions during the insertion of CVCs, cleaning the skin with chlorhexidine, avoiding femoral site if possible, and removing unnecessary catheters. Lastly, the use of antibiotics, appropriateness of therapy, and length of therapy were measured as supported by the current literature and were included among the other measures. 
The last of the tools developed to measure quality indicators was on fall prevention. The tool measured whether the patient was at risk and if so if it was properly identified. Also, the tool measured the use of restraints, whether there was an event or not, the assessment of patient level of consciousness and mentation (e.g., awake, oriented) and if measures like bed side-rails up and call buttons on reach were observed. In addition to the described tools, Microsoft ${ }^{\circledR}$ PowerPoint ${ }^{\circledR}$ presentations were developed as complementary teaching instruments, to provide bedside nurses with information regarding each specific bundle requirements and to reinforce adherence to bundle recommendations. T1 coincides with the highest number of APN Intensivists employed in the unit (i.e.; 14) which may have facilitated the allocation of APNs' resources for this task.

For T2 (January 2010 through June 2010) APN Intensivists began an extensive awareness campaign to encourage and enforce hand hygiene among healthcare workers and to extend this to family members and visitors to the SICU. This campaign was the preamble to implementing the special initiatives described above and lead to establishing target patient outcomes and adherence to implementation of the adopted guidelines and protocols (bundles) specific to each outcome. During this time period, APN Intensivists started using the tools and data were collected for the months of May and June. The task of data collection was shared initially among APN Intensivists working day and night shifts. One day time APN Intensivist was designated shift leader and the same occurred for the night shift staff. This decision was made to ensure data were collected appropriately and in a timely manner and to provide support and guidance to APN 
Intensivists in charge of data collection. The data base was established and educational support was provided to staff along data collection.

For T3 (July 2010 through December 2010) the data collection and education was delegated to the night shift APN Intensivist for the entire SICU. In addition, a pilot program was established during this time period to educate bedside nurses in obtaining APACHE scores for new admits to the unit (not done customarily in the unit until implementation of pilot program). The designated data collecting APN Intensivist, in addition to their regular clinical duties, was also responsible for APACHE-scoring on patients admitted to the SICU over a 24-hour time frame independently from whether the patient was admitted during their shift or not.

For T4 (January through December 2011) enforcement of the APN Intensivist's special initiatives were progressively decreased. The unit no longer counted on the availability of an APN Intensivist to enforce protocols, implement surveillance, and collect data as intended with the establishment of these initiatives among surgical patients admitted to the SICU. However during this period of time the unit's administration continued enforcing the recommendations dictated by the guidelines and practice bundles and maintained the implementation established over the previous time periods by the APN Intensivists.

During T4 the APN Intensivists assigned to cardiothoracic surgical patients (a distinctive group of patients admitted to the SICU) began to develop and implement a tool to measure post-surgical glycemic control within that group. Because hyperglycemia is associated with mortality of ICU patients (Krinsley, 2008), the implementation of surveillance efforts for this measure and the development of tools to capture data using a 
tool that identifies standards of care for this purpose was of upmost importance.

Glycemic indexes (measures) were recorded on post-surgical cardiothoracic patients for the first 24 and 48 hours of admission to the SICU. The selected measure was a glycemic index reported at 0600. Additional demographic information including the medical record number, type of surgery, and admission time to SICU were also included.

In addition to the aforementioned initiatives developed and implemented by these SICU's APN Intensivists it is important to mention that these APN Intensivists were also engaged in submitting abstracts to local, national, and international forums.

Disseminating findings and showcasing the role of APN Intensivists in ICU settings were also initiatives that resulted in recognition from peers and collaborating partners and contributed to advance the knowledge of the role of APNs in this field. 


\section{CHAPTER V}

\section{Discussion}

Research has identified and extensively documented the contributions of APNs on patient outcomes and healthcare systems. However, while current literature documents the need for research into alternative methods of ICU healthcare delivery the lack of supporting evidence on the effects of APNs as Intensivists remains. Despite the magnitude of ICU complications, there is a dearth of research documenting APN Intensivists' effects in generating outcome-driven care indicators, demonstrated reductions in length of stay, decreases in costs, and prevention of healthcare-associated complications. In light of current legislation, input substitution (i.e., allowing the substitution of NPs for MDs) as an alternative in providing care (Laurant et al., 2006) and policies to find cost effective alternative models of healthcare delivery, there is great need to document the effects of this APN intensivists model of ICU staffing. The growing aging population, physician intensivist attrition, and the rise of healthcare costs provide further need for this research. The purpose of this study was to examine the dose effect of APN Intensivists in a surgical intensive care unit (SICU) on patient outcomes, healthcare charges, APN Intensivists services, and APNs special initiatives when APN staffing differed.

\section{Healthcare-Associated Infections (HAIs)}

In the present study differences were found in patient outcomes (HAIs [i.e., VAP, CAUTI], pressure ulcers, post-surgical glycemic control, ventilator days, urinary catheter days and length of stay [LOS]) when the ICU APN staffing differed. The mean number of ventilator days in the study was $2,226.5$ days, ranging from 1,557 days in $\mathrm{T} 1$ to 1,763 
days in $\mathrm{T} 2$ to 1,219 days in $\mathrm{T} 3$ and to 4,367 days in T4. The increased number of ventilator days from T3 $(1,219)$ when the unit was staffed with 10 APNs to 4,367 in T4 when there were 7 APNs may potentially be attributed to the decrease in the number of APNs during these time periods.

The VAP rates, calculated by dividing the number of infections (i.e., VAP) by the number of ventilator days and multiplying that result by 1000 for every time period, ranged from $0.68 \times 1000$ ventilator days at $\mathrm{T} 4$ to $3.40 \times 1000$ ventilator days at $\mathrm{T} 2$. These results may seem paradoxical, however, they may reflect the implementation and adherence to the VAP bundle developed and implemented by APNs beginning at T2 This adherence and monitoring may be responsible for the VAP rate decreasing to its lowest at $\mathrm{T} 4$ despite the increased number of ventilator days at $\mathrm{T} 4$.

These results are consistent with those of recent studies reporting rates of VAP from 1 to 4 cases per 1,000 ventilator-days, although it may exceed 10 cases in high risk populations such as surgical ICU patients (Coffin et al., 2008). Research has demonstrated that targeting modifiable risk factors can decrease rates of postoperative pneumonia. Implementing measures such as minimizing the risk of aspiration by elevating the head of the bed at least 30 degrees; (American Thoracic Society [ATS], 2005; Kieninger \& Lipsett, 2009; Metheny et al., 2006), elimination of colonizing organisms in the upper aerodigestive tract by providing good oral hygiene and the use of chlorhexidine gluconate rinse (Kieninger \& Lipsett, 2009; Segers, Speekenbrink, Ubbink, van Ogtrop, \& de Mol, 2006); decontamination of the aerodigestive tract; stress ulcer prophylaxis that may decrease morbidity and mortality (Cook et al., 2001; Dodek et al., 2004); management of the mechanical airway system including the endotracheal tube and 
the ventilator circuit (Kieninger \& Lipsett, 2009); elimination of secretions pooling on the ETT cuff and the consequent reduction of tracheal contamination (Kieninger \& Lipsett, 2009; Sole et al., 2002); semi-recumbence position (Drakulovic et al., 1999; Van Nieuwenhoven et al., 2006; Grap et al., 2003; Grap et al., 2005); frequent change of suctioning equipment (Sole et al., 2002), and preventing the pooling of subglottic secretions (Bouza et al., 2008). Implementation of these measures requires development of policies and procedures for adhering to and monitoring of compliance by providers. This study documented the leadership in this area by the APNs.

The number of catheter days in T1 $(3,193)$ and T2 $(3,342)$ were similar when the number of APNs were 14 and 13 respectively. Catheter days for T4 climbed to 6882 when there were 7 APNs from 2868 when there were 10 APNs. This could potentially be due to an inability of the staff to remove the urinary catheters at an appropriate time, or a changing patient severity. The number of catheter-associated urinary tract infections (CAUTI), however, were low and ranged from four cases in T3 to 11 cases in T4, again showing an increase with the lower numbers of APNs at T4. The CAUTI rates are calculated by dividing the number of infections (i.e., CAUTI) by the number of urinary catheter days and multiplying that result by 1000 . These findings in increased catheter days and catheter-associated urinary tract infections (CAUTI) may be attributed to the dose effect of APNs and their progressively decreased numbers from T1 to T4.

The findings from the present study on CAUTI are consistent with those described by Brooten \& Naylor (2007) on nurse dose and effects on patient outcomes. In that respect the present study findings may add to our knowledge of APNs Intensivists effects on CAUTI by providing data that were not found in the literature. The number of 
CAUTIs, the number of catheter days and the catheter rate decreased from T1 through T3 and catheter days and CAUTIs increased at T4 when the number of APNs was lowest (7). These findings may demonstrate the effects of bundles aimed to decrease CAUTI incidence as documented in many studies (Elpern et al., 2009; APIC, 2008; Fakih et al., 2008; Huang et al., 2004; (Lo et al., 2008; Maki \& Tambyah, 2001; (Huang et al., 2004; Rosenthal, Guzman, and Safdar, 2004, Gould et al., 2009; Lo et al., 2008).

\section{Pressure Ulcers}

Pressure ulcers are considered to be one of the most important indicators of the quality and effectiveness of patient care (Robinson, 2005). The study's pressure ulcers data reflected the incidence of unit acquired pressure ulcers of stage II and above and the pressure ulcer prevention measures implemented in SICU by APNs Intensivists.

Patients in the ICU have a higher probability for developing pressure ulcers (1.5 times higher) (Lahmann et al., 2012). The findings of the Lahmann et al., (2012) study suggested that patient specific factors and implementation of preventive interventions contribute to the higher rate of pressure ulcers incidence in the ICUs. Therefore, increasing awareness and providing adequate interventions in the ICU may help in decreasing the occurrence of pressure ulcers in the ICU population (Lahmann et al., 2012).

When comparing the incidence of pressure ulcers in the first two quarters of $\mathrm{T} 1$ $(28.1 \%)$ and the first two quarters of T2 (30. $2 \%)$ versus the first two quarters of T4 (35.7 \%) incidence was increased $(+7.6 \%$ and $+5.5 \%$ respectively) in $\mathrm{T} 4$. When comparing the incidence of pressure ulcers in the second two quarters of T1 (33.4\%) and pressure ulcers in the second two quarters of T3 (37.1\%) versus the second two quarters 
of T4 (31.6\%) the incidence was decreased (-1.8\% and $-5.5 \%$ respectively) in T4. Comparing pressure ulcer prevention measures implemented in the SICU by APN Intensivists to reduce the incidence of pressure ulcers is a next important step.

Published reports of the incidences of ICU pressure ulcers range from one to $56 \%$ (Keller et al., 2002; Jiricka, Ryan, Carvalho, \& Bukvich., 1995; Schoonhoven et al., 2002). The main reasons behind the increased incidence of pressure ulcers in ICU patients are immobilization, sedation, and the use of mechanical ventilation (Defloor et al., 2005). ICU patients may also, suffer from impaired circulation (especially the geriatric population) or the use of vasoactive drugs which increases the risk of developing pressure ulcers (Keller et al., 2002).

Shahin, et al. (2009) assessed the allocation of preventive measures for patients at risk of developing pressure ulcers and examined the evidence of applied preventive measures in the ICU according to the European Pressure Ulcer Advisory Panel (EPUAP) and Agency for Health Care Policy and Research (AHCPR). The findings of this study showed that $83 \%$ of patients were at risk for developing pressure ulcers using the Braden scale. Preventive measures used were pressure reducing devices including special mattresses (36.5\%), special beds (2.5\%), and special cushions (17.6\%). Nursing interventions that were implemented included repositioning (41.5\%), mobility (56.6\%), skin inspection (81.8\%), massage with moisturizer cream (80.5\%), avoidance of nutritional and fluid deficit (68.6\%), patient education (40.3\%) and family education (20.8\%), avoidance of sheer and friction (32\%), and massage (8.8\%). Mattresses (i.e., alternating pressure air, low air loss, and foam) were the most used preventive measures in at risk patients (Shahin et al., 2009). 
Patients who were identified to be at risk for developing pressure ulcers were found to have more than one nursing intervention implemented (Shahin et al., 2009). The findings documented that there was a significant difference in the allocation of preventive measures between the at-risk and not at-risk patient groups for pressure ulcer. The pressure ulcer preventive measures used were in congruence with those identified by the EPUAP and AHCPR guidelines with the exception of massage that was used with 8.8\% of patients. Although major limitations of this study were the sample, exclusion of unconscious patients, and the small size of the sample (Shahin et al., 2009) the enforcement of preventive measures is supported by this and other studies correlating pressure ulcers incidence with preventive measures such as nursing assessment (Compton et al., 2008). It is challenging to compare ICU patient populations and the incidence of pressure ulcers in these studies. The incidence of pressure ulcers at $\mathrm{T} 4$ in the present study as the number of APNs decreased is concerning and may warrant further exploration.

\section{Post-Surgical Glycemic Control}

Unit data for post-surgical glycemic control was measured by comparisons of fallouts (i.e., glucose values over $200 \mathrm{mg} / \mathrm{dl}$ ) in each of the study time periods. Data for $\mathrm{T} 1$ and T2 were incomplete. Data for T3 and T4 were complete and available for abstraction. Of the 43 cardiothoracic patients admitted to SICU at T2 only 2 (4.7\%) fallouts were recorded. For T3 13 (9.4\%) fallouts from 139 admissions were recorded. For T4 18 (6.8\%) fallouts from 263 admissions were recorded.

Because data on $\mathrm{T} 1$ were incomplete, it was not possible to compare post-surgical glycemic control outcomes between T1 vs. T4. When comparing data on T2 (two months 
reported) versus $\mathrm{T} 4$ the percentage of fallouts was lower for T2 (4.7\%) than for T4 (6.8\%). When comparing post-surgical glycemic control at $\mathrm{T} 3$ versus $\mathrm{T} 4$ the percentage of fallouts was greater in T3 (9.4\%) than for T4 (6.8\%). However, no statistically significant differences between the mean numbers of glucose fallouts were found between $\mathrm{T} 3$ and $\mathrm{T} 4$.

Hyperglycemia has been associated with increased mortality in critically ill patients (Krinsley, 2008). Controlling a low degree of glycemic variability may significantly decrease mortality and improve patient outcomes in ICUs implementing glycemic variability metric (Krinsley, 2008). The optimal target goals of glucose levels in ICU patients remain uncertain (Kavanagh \& McCowen, 2010). Recently, the American Association of Clinical Endocrinologists and American Diabetes Association recommended target glucose levels for ICU patients between 140 to $180 \mathrm{mg} / \mathrm{dl}$ (ADA, 2010).

Other organizations (i.e., Surviving Sepsis Campaign [SSC], Institute for Healthcare Improvement [IHI], 2011b) suggested target glucose levels of $150 \mathrm{mg} / \mathrm{dl}$ to less than $180 \mathrm{mg} / \mathrm{dl}$ respectively. Further evidence of the importance of post-surgical glycemic control have been well supported by studies examining the effect of an intensive glycemia management protocol on the cost of care of critically ill adult patients suggesting a net annual decrease of $\$ 1,339,500$ or $\$ 1,580$ per patient (Krinsley \& Jones, 2006). These findings showed a decrease in ICU LOS, ventilator days, costs (i.e., laboratory, pharmacy, and radiology), and post-ICU hospital LOS (Krinsley \& Jones, 2006). Despite the controversies surrounding management of glycemic control in the ICU population, interventions aiming to controlling glycemia in surgical ICU patients 
have also demonstrated reduced hospital-acquired infections, need for dialysis, and postoperative complications (Grey \& Perdizet, 2004). Although there were no statistically significant differences found between the mean numbers of glucose fallouts in comparing study time periods the importance of controlling glycemia in surgical ICU patients remains important for further study.

\section{Length of Stay (LOS)}

The length of stay (LOS) for patients in the SICU varied across time periods ranging from a mean stay of 4.5 days $(\mathrm{SD}=5.88)$ at $\mathrm{T} 1$ to 3.9 days $(\mathrm{SD}=4.09)$ at $\mathrm{T} 2,7.2$ days $(\mathrm{SD}=13.0)$ at $\mathrm{T} 3$ and 7.7 days $(\mathrm{SD}=15.4)$ at $\mathrm{T} 4$. These data again show an increasing LOS at T3 and especially at T4 when the number of APNs was lowest.

Williams and colleagues (2010) examined the clinical and mortality data of 22,298 adult ICU patients over a 5 year period. The median ICU LOS was 17 days. The majority of the patients staying more than 10 days in the ICU were either septic or trauma victims. ICU LOS contributed to the long term mortality after hospital discharge after adjusting for other risk factors (Williams et al., 2010). Wise and colleagues (2011) compared mortality and LOS of medical ICU patients cared for by a hospitalist and an intensive-led team. The mean ICU LOS for the total sample was 4 days $(\mathrm{SD}=5.9)$ and the mean hospital LOS was 9.1 days $(\mathrm{SD}=9.0)$.

Kramer and Zimmerman (2010) developed and validated a model that identified patients at risk for prolonged ICU LOS. The retrospective cohort study reviewed a total of 343,555 charts of admissions to 83 ICUS in 31 U.S. hospitals. The ICU LOS was reviewed and a five day cut point was identified as a time-point after which prolonged LOS was a concern. The five day cut point was chosen because it was suggested to allow 
time to show complications and responses to therapy. Patients with more than five days ICU LOS were found to have significantly higher severity of illness, frequency of mechanical ventilation, emergency surgery, and ICU readmissions. These patients had poorer outcomes and accounted for $21 \%$ of ICU admissions and $63 \%$ of total ICU days.

While it is difficult to compare the ICU patient populations and the LOS in these studies, the increasing LOS to 7.7 days at T4 in the present study as the number of APNs decreased is concerning, is above the 5 day ICU LOS noted by Kramer and Zimmerman (2010) and may suggest an increasing patient severity at a time of decreased number of APN Intensivists.

\section{Healthcare Charges for SICU Length of Stay and APN Intensivists' Services}

Total healthcare charges for each of the four study periods (T1-T4), for SICU patient stays for the sample were not available to the PI. It is crucial to recognize the costs of patient conditions (e.g., HAIs, pressure ulcer) and distinguish their impact on the micro-cost or the resources used for the treatment of these conditions and the hospital charges that are incurred from patient care when studying the economic burden of these patient conditions. Ideally, the most accurate analysis would be to use micro-costs since they reflect the actual cost of the services and treatments provided. Unfortunately, information on the micro-costs may not be feasible to extract in most instances and therefore total hospital charges are used as an alternative in many studies and published reports. There are also intangible costs for patient conditions, costs that may have equal or greater burden than the financial costs. For instance, Scott (2009) defines these intangible costs as part of the social costs of HAIs, namely psychological cost (i.e., anxiety, grief, job loss, ensuing disabilities), pain and suffering, and change in social 
functioning and activities of daily living. There is a dearth of literature documenting these intangible costs as a result of HAIs and pressure ulcers in the ICU.

In the present study charges for APN Intensivists' services reflected charges attributed to SICU APN Intensivists procedures from the inception of billing for services in the SICU in 2009 to December 2012. Total healthcare charges for APN Intensivists' services for the sample were $\$ 90,478$. In T1 charges were $\$ 11,268$, in T2 they were $\$ 18,691$ in $\mathrm{T} 3$ they were $\$ 8,792$ and in T4 they were $\$ 51,727$. The increased charges for APN services at $\mathrm{T} 4$ could be attributed to a change in the system for capturing charges for APN services. Prior to 2012 billing for APN services was inconsistent and done manually. In 2012 a system was put in place to capture APN billing electronically.

There is ample literature documenting improved patient outcomes and reductions in health care costs of APN services. The ability to bill for APN Intensivists ICU services may be very important for many health care institutions. In the present study's setting medical interns (PG1-PG3) are not allowed to charge for procedures that are not supervised by either a fellow or attending physician. In fact, for any invasive procedure performed in the SICU an attending physician is advised to be present to supervise the procedure and ensure patient safety and the proper capture of revenues. However, trained APN Intensivists are allowed to perform such procedures with the consent of the attending physician independently and allowed to bill as independent providers. This is particularly significant for APN Intensivists in the SICU since they are able to capture revenues without the physical presence on an attending physician, especially on the night shifts when the physical presence of attending physicians is decreased. The present study 
findings add to our knowledge of billing for APN services in the SICU by providing data that were not found in the literature.

\section{Frequency of APN Intensivist Special Initiatives}

In the present study there was a difference in the frequency of APN Intensivist special initiatives initiated and conducted over the four time periods. In T1 when there were 14 APNs, the APNs established themselves as a team. While no initiatives were begun during this period, the team worked on identifying targeted initiatives. In T2 when there were 13 APNs three initiatives were established. In T3 with 10 APNs there were three initiatives ongoing and no new initiatives were begun. In T4 blood glucose monitoring was continued from T2 and only one new initiative, the cardiac admission checklist, was begun. Development and monitoring of these special initiatives may well have been the reason for the decrease in VAP rates and CAUTI rates in the SICU for this sample.

As the number of APNs decreased APN initiatives were not carried out consistently for APACHE II scores, falls bundle, antibiograms, and the APN database. The lack of follow up and consistent monitoring of these measures may have a negative impact on patient outcomes. Further studies are needed to evaluate the impact of this lack of consistent monitoring. The present study findings add to our knowledge of the effects of APN Intensivists on APN's initiatives in a SICU by providing data that were not found in the literature.

The findings on patient outcomes by monitoring patient condition are consistent with the research of Pronovost et al. (1999) and that of Gajic et al. (2008). Using a multitiered, multivariate analysis the study showed that daily rounds in the ICU by an ICU 
physician (intensivist) was associated with reduced in-hospital mortality and reduced postoperative medical complications. A systematic review was conducted by Pronovost, Young, Dorman, Robinson and Angus (1999) of the available literature on physician staffing and outcomes; the authors concluded that there was a consistent finding of decreased mortality and decreased length of stay with intensivist presence in the ICUs. Gajic et al. (2008) reported, with the introduction of continuous (24 hours) on-site presence of an intensivist there was improved processes of care, staff satisfaction, decreased complications, and reduced length of stay. The rate of complications decreased from $11 \%$ to $7 \%$ per patient-day. The introduction of night coverage in the ICU was responsible for these differences and documented the evidence that intensivists' presence in ICUs is beneficial for patients, families, and overall healthcare (Gajic et al., 2008). These results may be consistent with the present study results of decreased VAP rates and CAUTI rates in the SICU for this sample.

\section{Study Limitations}

The limitations in this study are common in studies of this type where data are abstracted from health care systems where methods of data collection may change over the time of the study and the data are limited to the data recorded and available to the investigator. There were several study limitations that may affect the generalizability of the findings and potential results. Data on the number of staff nurses practicing during the study time period were not available and could have affected the results. The study was conducted in a SICU composed predominantly of post-surgical liver and kidney and pancreas transplant patients. It is not known if the findings would hold in samples with different admission diagnoses. The generalizability of these findings may not apply to 
other SICU's patient populations. In addition, the unavailability of individual APACHE scores for the sample limited the anchoring of findings to patients' acuity levels.

Data abstraction for 816 charts became problematic due to changes in the health system's methods of documentation and data collection for key outcomes, major changes in the system and in its leadership, and several changes in key personnel providing access to the study data. There were missing data on patient outcomes in the charts necessitating use of aggregate data. Data on pressure ulcers were obtained from reports generated by JMH to the National Data of Quality Indicators (NDQI). Since these data were reported as aggregate data, the mean incidence of pressure ulcers in the SICU for the study's timeperiods could not be reported. Similarly, data on HAIs (VAP, CAUTI) were obtained from aggregate data and presented similar limitations.

Data on post-surgical glycemic control were obtained from a database managed by the APN Intensivists. These data were limited to the time periods where these measures were collected and did not capture all of the study's four time-periods. APN Intensivists' charges data were reported as aggregate measures and could not be correlated to specific procedures.

\section{Policy Implications}

Despite evidence suggesting the use of high-intensity intensivist staffing to improve patient outcomes and reduce ICU associated costs $73 \%$ of the ICUs in this country provide low-intensity intensivist staffing or no intensivist care coverage at all (Gajic \& Afessa, 2009). Over 5 billion dollars a year could be saved in healthcare costs by implementing changes of physician intensivist staffing just in non-rural U.S. hospitals (Pronovost et al., 1999). However, the shortage of intensivists and failure to implement 
the Accreditation Commission of Colleges on Medicine (ACCM) guidelines for ICU staffing may prevent not only saving these billion dollar healthcare costs but lives as well. New ICU staffing paradigms are needed to provide safe, cost-effective, and suitable care to critically ill patients.

Evidence supporting the impact of intensivists and the Intensivist Team Model (intensivist-lead multidisciplinary team) in providing best patient outcomes (Durbin, 2006), points to the potential contributions of APN Intensivists to this team model in ICUs. In contrast to the attrition of physician intensivists and critical care trained physicians, NPs are increasing in number within the field of acute care specialties, both in the United States and globally. The evolution of the role of ACNPs in acute care has responded to an increased need of tertiary services, the shortage of medical staffing to respond to these needs, and the advancement of the role of ACNPs in clinical practice. In intensive care units (ICUs), the role of ACNPs is evolving to alleviate the physician intensivist shortage and in some instances replace physicians and support patient care.

Findings of this study reinforce the need for further research to support the development and implementation of policies that will help improve patient outcomes, decrease healthcare costs, and advance APN Intensivists practice to the forefront of rendering affordable and effective healthcare.

\section{Implications for Future Research}

Although findings from this study were limited in generalizability, the effects of APN Intensivists in critical care and intensive care units on patient outcomes and health care costs must continue to be explored. The information on the effects of this APN model on patient outcomes, and healthcare costs, should guide future research endeavors. 
This is particularly true in the area of healthcare associated infections (HAIs), pressure ulcers, length of stay, and other quality indicators of care in intensive care units.

Future research may include comparing patient outcomes against medical and surgical diagnoses, length of stay, and costs. Another needed study is an examination of data on different HAIs (i.e., Central Line Bloodstream Infections [CLABSI]) in the same setting or transitionally. And methodologically, future research using patient chart review may yield more specific and rigorous data than that provided in the aggregate in existing data bases. 


\section{APPENDICES}

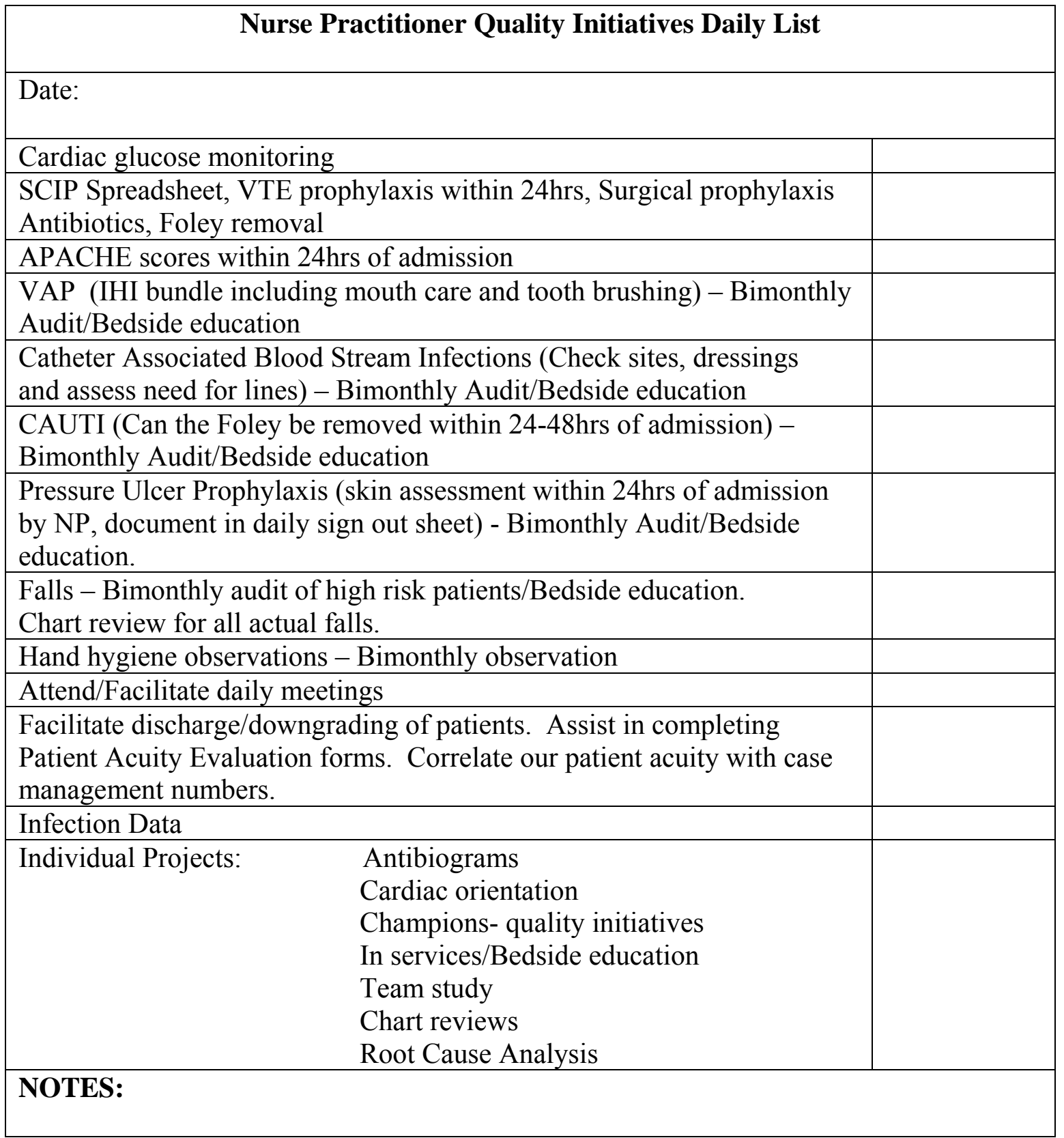

Appendix 1. Nurse Practitioner Quality Initiatives Daily List 


\section{REFERENCES}

Accreditation Council for Graduate Medical Education (2004). The ACGME's approach to limit resident duty hours 12 months after implementation: A summary of achievements: 2004. Retrieved from http://www.acgme.org/acgmeweb/Portals/0/PFAssets/PublicationsPapers/dh_duty hoursummary2003-04.pdf

Ackerman, M. H. (1997). The acute care nurse practitioner: evolution of the clinical nurse specialist? Heart Lung, 26 (2), 85-86.

Ackerman, M. H., Norsen, L., Martin, B., Wiedrich, J., \& Kitzman, H. J. (1996). Development of a model of advanced practice. American Journal of Critical Care, 5(1), 68-73.

Aiken, L. H., Clarke, S. P., Cheung, R. B., Sloane, D. M., \& Silber, J. H. (2003). Educational levels of hospital nurses and surgical patient mortality. JAMA, 290 (12), 1617-1623.

Aiken, L. H., Clarke, S. P., Sloane, D. M., Sochalski, J., \& Silber, J. H. (2002). Hospital nurse staffing and patient mortality, nurse burnout, and job dissatisfaction. JAMA, 288(16), 1987-1993.

Aiken, L. H., Lake, E. T., Semaan, S., Lehman, H. P., O'Hare, P. A., Cole, C. S., ... Frank, I. (1993). Nurse practitioner managed care for persons with HIV infection. Journal of Nursing Scholarship, 25(3), 172-177.

Alonso-Echanove, J., Edwards, J. R., Richards, M. J., Brennan, P., Venezia, R. A., Keen, J., ...Gaynes, R. P. (2003). Effect of nurse staffing and antimicrobial-impregnated central venous catheters on the risk for bloodstream infections in intensive care units. Infection Control and Hospital Epidemiology, 24(12), 916-925. doi: $10.1086 / 502160$

Amaravadi, R. K., Dimick, J. B., Pronovost, P. J., \& Lipsett, P. A. (2000). ICU nurse-topatient ratio is associated with complications and resource use after esophagectomy. Intensive Care Medicine, 26(12), 1857-1862. doi: $10.1007 / \mathrm{s} 001340000720$

Amba, K. T. (2011, January/February). Acute care NPs have new opportunities in virtual ICUs. Nurse Practitioners World News, 16(1/2). Retrieved from http://www.icumedicine.com/pdfs/VirtualICUNPWN2011.pdf

American Academy of Nurse Practitioners (AANP). (2010a). Nurse Practitioner facts. Retrieve from http://www.aanp.org/images/documents/research/20102011np\%20facts2012.pdf 
American Academy of Nurse Practitioners (AANP). (2010b). Scope of practice for Nurse Practitioners. Retrieved from http://ndnpa.org/2010ScopeOfPractice1[1].pdf

American Academy of Nurse Practitioners (AANP). (2010c). Nurse Practitioner prescriptive privilege. Retrieved from https://www.aanp.org/images/documents/publications/prescriptiveprivilege.pdf

American College of Nurse Practitioners. (n.d.-a).Nurse Practitioner scope of practice. Retrieved from http://www.acnpweb.org/i4a/pages/index.cfm?pageid=3465

American College of Nurse Practitioners. (n.d.-b). What is a nurse practitioner? Retrieved from http://www.acnpweb.org/files/public/What_is_a_Nurse_Practitioner.pdf

American Diabetes Association (ADA). (2010). Standards of Medical Care in Diabetes. Diabetes Care, 33(Suppl. 1), S11-S61. doi: 10.2337/dc10-S011

American Thoracic Society (ATS). (2005). Guidelines for the management of adults with hospital-acquired, ventilator-associated, and healthcare-associated pneumonia. American Journal of Respiratory and Critical Care Medicine, 171, 288-416. doi: 10.1164/rccm.200405-644ST

Angus, D. C., Kelley, M. A., Schmitz, R. J., White, A., \& Popovich, J., Jr. (2000). Current and projected workforce requirements for care of the critically ill and patients with pulmonary disease. Can we meet the requirements of an aging population? JAMA, 284(21), 2762-2770.

APRN Joint Dialogue Group. (2008). Consensus model for APRN regulation: Licensure, accreditation, certification and education. Retrieved from http://www.aacn.nche.edu/education/pdf/APRNReport.pdf

Association for Professionals in Infection Control and Epidemiology (APIC). (2008). Guide to the Elimination of Catheter-Associated Urinary Tract Infections (CAUTIS). Retrieved from: http://www.apic.org/Resource_EliminationGuideForm/c0790db8-2aca-4179a7ae-676c27592de2/File/APIC-CAUTI-Guide.pdf

Beale, R. J., Bryg, D. J., \& Bihari, D. J. (1999). Immunonutrition in the critically ill: A systematic review of clinical outcome. Critical Care Medicine, 27(12), 27992805 .

Becker, D., Kaplow, R., Muenzen, P.M., \& Hartigan, C. (2006). Activities performed by acute and critical care advanced practice nurses: American Association of Critical Care Nurses study of practice. American Journal of Critical Care, 15(2), 130-148. 
Bennett, L., Kavner, D., Lee, B. Y., Trainor, F. S., \& Lewis, J. M. (1981). Skin blood flow in seated geriatric patients. Archives of Physical Medicine and Rehabilitation, 62(8), 392-398.

Berthelsen, P. G., \& Cronqvist, M. (2003). The first intensive care unit in the world: Copenhagen 1953. Acta Anaesthesiologica Scandinavica, 47(10), 1190-1195.

Blegen, M. A., Goode, C. J., \& Reed, L. (1998). Nurse staffing and patient outcomes. Nursing Research, 47(1), 43-50.

Bigatello, L. M., Stelfox, H. T., Berra, L., Schmidt, U., \& Gettings, E. M. (2007). Outcome of patients undergoing prolonged mechanical ventilation after critical illness. Critical Care Medicine, 35(11). 2491-2497. doi: 10.1097/01.CCM.0000287589.16724.B2

Binnekade, J. M., Vroom, M. B., de Mol, B. A., \& de Haan, R. J. (2003). The quality of intensive care nursing before, during, and after the introduction of nurses without ICU training. Heart Lung, 32(3), 190-196.

Birkmeyer, J. D., \& Dimick, J. B. (2004). The Leapfrog Group's patient safety practices, 2003: The potential benefits of universal adoption. The Leapfrog Group, 1-45. Retrieved from: http://leapfroggroup.org/media/file/Leapfrog-Birkmeyer.pdf

Birkmeyer, J. D., Dimick, J. B., \& Birkmeyer, N. J. O. (2004). Measuring the quality of surgical care: Structure, process, or outcomes? Journal of the American College of Surgeons, 198(4), 624-632. doi: 10.1016/j.jamcollsurg.2003.11.017

Bochicchio, G. V., \& Scalea, T. M. (2008). Glycemic control in the ICU. Advances in Surgery, 42, 261-275. doi:10.1016/j.yasu.2008.03.006

Bouza, E., Burillo, A., \& Muñoz, P. (2008). Empiric therapy for intravenous central line infections and nosocomially-acquired bacterial endocarditis. Critical Care Clinics, 24(2), 293-312. doi: 10.1016/j.ccc.2007.12.002

Brilli, R.J., Spevetz, A., Branson, R.D., Campbell, G. M., Cohen, H., Dasta, J. F., ... the members of the American College of Critical Care Medicine Guidelines for the Definition of an Intesivist and the Practice of Critical Care Medicine (2001). Critical care delivery in the intensive care unit: Defining clinical roles and the nest practice model. Critical Care Medicine, 29(10), 2007-2019.

Brook, A., Ahrens, T. S., Robyn, S., Prentice, D., Glenda, S., William, S., Shannon, W., $\&$ Kollef, M. (1999). Effect of a nursing-implemented sedation protocol on the duration of mechanical ventilation. Critical Care Medicine, 27(12), 2609-2615. 
Brook, A. D., Ahrens, T. S., Schaiff, R., Prentice, D., Sherman, G. Shannon, W. \& Kollef, M. H. (1999). Effect of a nursing-implemented sedation protocol on the duration of mechanical ventilation. Critical Care Medicine, 27(12), 2609-2615.

Brooten, D., Kumar, S., Brown, L. P., Butts, P., Finkler, S. A., Bakewell-Sachs, S., ... Delivoria-Papadopoulos, M. (1986). A randomized clinical trial of early discharge and home follow-up of very-low-birth-weight infants. The New England Journal of Medicine, 315(15), 934-939.

Brooten, D., Naylor, M. D., York, R., Brown, L. P., Hazard Munro, B., Hollingsworth, A. O., ... Youngblut, J. M. (2002). Lessons learned from testing the quality cost model of advanced practice nursing (APN) transitional care. Journal of Nursing Scholarship 34(4), 369-375. doi: 10.1111/j.1547-5069.2002.00369.x

Brooten, D., Roncoli, M., Finkler, S., Arnold, L., Cohen, A., \& Mennuti, M. (1994). A randomized trial of early hospital discharge and home follow-up of women having cesarean birth. Obstetrics and Gynecology, 84(5), 832-838.

Brooten, D., Youngblut, J. M., Brown, L., Finkler, S. A., Neff, D. F., \& Madigan, E. (2001). A randomized trial of nurse specialist home care for women with highrisk pregnancies: Outcomes and costs. American Journal of Managed Care, 7(8), 793-803.

Brooten, D., Youngblut, J. M., Deatrick, J., Naylor, M. \& York, R. (2003). Patient problems, Advanced Practice Nurse (APN) interventions, time and contacts among five patient groups. Journal of Nursing Scholarship, 35(1), 73-79.

Brooten, D., Youngblut, J. M., Deosires, W., Singhala, K., \& Guido-Sanz, F. (2011). Global considerations in measuring effectiveness of advanced practice nurses. International Journal of Nursing Studies. 49(7), 906-912. Retrieved from http://dx.doi.org/10.1016/j.ijnurstu.2011.10.022

Brooten, D., Youngblut, J. M., Donahue, D., Hamilton, M., Hannan, J., \& Neff, D. F. (2007). Women with high-risk pregnancies, problems, and APN interventions. Journal of Nursing Scholarship, 39(4), 349-357. doi: 10.1111/j.15475069.2007.00192.x

Brosnahan, J. Jull, A., \& Tracy, C. (2004). Types of urethral catheters for management of short-term voiding problems in hospitalized adults. Cochrane Database of Systemic Reviews, Issue 2. Update Software; Oxford.

Brown, J., Doloresco F., III, \& Mylotte, J. M. (2009). "Never Events": Not every hospital-acquired infection is preventable. Clinical Infectious Diseases, 49(5), 743-746. doi: 10.1086/604719 
Brown, J. J., \& Sullivan, G. (1989). Effect on ICU mortality of a full-time critical care specialist. Chest, 96(1), 127-129.

Bryant-Lukosius, D., DiCenso, A., Browne, G., \& Pinelli, J. (2004). Advanced practice nursing roles: Development, implementation, and evaluation. Journal of Advanced Nursing, 48(5), 519-529. doi:: 10.1111/j.1365-2648.2004.03234.x

Bullough, B. (1992). Alternative models for specialty nursing practice. Nursing and Health Care, 13(5), 254-259.

Bupert, C. (2004). What is a nurse practitioner? In Nurse Practitioner's Business Practice and Legal Guide (2 ${ }^{\text {nd }}$ ed.). Boston: Jones \& Bartlett. pp. 4-6.

Burchardi, H., \& Moerer, O. (2001). Twenty-four hour presence of physicians in the ICU. Critical Care, 5(3), 131-137.

Burns, S. M., Earven, S., Fisher, C., Lewis, R., Merrell, P., Schubart, J. R., ... University of Virginia Long Term Mechanical Ventilation Team. (2003). Implementation of an institutional program to improve clinical and financial outcomes of mechanically ventilated patients: One-year outcomes and lessons learned. Critical Care Medicine, 31(12), 2752-2763.

Cajulis, C. B., \& Fitzpatrick, J. J. (2007). Levels of autonomy of nurse practitioners in an acute care setting. Journal of the American Academy of Nurse Practitioners, 19(10), 500-507. doi: 10.1111/j.1745-7599.2007.00257.x

Carlson, S. S., Stocking, C., Podsadecki, T., Christenson, J., Pohlman, A., MacRae, S., ... Hall, J. (1996). Effects of organizational change in the medical intensive care unit of a teaching hospital: A comparison of open and closed formats. JAMA, 276(4), $322-328$.

Carrion, M. I., Ayuso, D., Marcos, M., Robles, M. P., de la Cal, M., Alia, I., \& Esteban, A. (2000). Accidental removal of endotracheal and nasogastric tubes and intravascular catheters. Critical Care Medicine, 28(1), 63-66.

Carson, S. S., Kress, J. P., Rodgers, J. E., Vinayak, A., Campbell-Bright, S., Levitt, J., ... $\&$ Hall, J. (2006). A randomized trial of intermittent lorazepam versus propofol with daily interruption in mechanically ventilated patients. Critical Care Medicine, 34(5), 1326-1332.

Chanques, G., Jaber, S., Barbotte, E., Violet, S., Sebbane, M., Perrigault, P. F., ... Eledjam, J. J. (2006). Impact of systematic evaluation of pain and agitation in an intensive care unit. Critical Care Medicine, 34(6), 1691-1699. 
Chastre, J., Wolff, M., Fagon, J. Y., Chevret, S., Thomas, F., Wermert, D., Clementi, E., ... PneumA trial group. (2003). Comparison of 8 vs 15 days of antibiotic therapy for ventilator-associated pneumonia in adults: A randomized trial. JAMA, 290 (19), 2588-2598. doi: 10.1001/jama.290.19.2588

Chen, L. M., Martin, C. M., Morrison, T. L., \& Sibbald, W. J. (1999). Interobserver variability in data collection of the APACHE II score in teaching and community hospitals. Critical Care Medicine, 27(9), 1999-2004.

Center for Disease Control Morbidity and Mortality Weekly Report (CDC MMWR). (2005). Guidelines for preventing Health-Care-Associated Pneumonia, 2003. Retrieved from: http://www.cdc.gov/mmwr/preview/mmwrhtml/rr5303a1.htm

Centers for Medicare and Medicaid Services. (2009). 100\% MEDPAR inpatient hospital national data for Fiscal Year 2009 short stay inpatient Diagnosis Related Groups. Retrieved from:

https://www.cms.gov/MedicareFeeforSvcPartsAB/Downloads/DRG09.pdf

Centers for Medicare and Medicaid Services (CMS), HHS. (2008). Medicare program; Changes to the hospital inpatient prospective payment system and fiscal year 2009 rates. Federal Register, 73(161), 48433-9084. Retrieved from http://edocket.access.gpo.gov/2008/pdf/E8-17914.pdf

Cho, S. H., Ketefian, S., Barkauskas, V. H., \& Smith, D. G. (2003). The effects of nurse staffing on adverse events, morbidity, mortality, and medical costs. Nursing Research, 52(2), 71-79.

Clarke, S. P., \& Aiken, L. H. (2003). Registered nurse staffing and patient and nurse outcomes in hospitals: A commentary. Policy, Politics \& Nursing Practice, 4(2), 104-111 74-84.

Coffin, S. E., Klompas, M., Classen, D., Arias, K. M., Podgorny, K., Anderson, D. J., ... Yokoe, D. S. (2008). Strategies to prevent ventilator-associated pneumonia in acute care hospitals. Infection Control and Hospital Epidemiology, 29(Suppl. 1), S31-S40. doi: 10.1086/591062

Coombs, M., Chaboyer, W., \& Sole, M. L. (2007). Advanced nursing roles in critical care-a natural or forced evolution? Journal of Professional Nursing, 23(2), 83-90. doi: 10.1016/j.profnurs.2006.07.003

Combes, A., Luyt, C. E., Fagon, J. Y., Wollf, M., Trouillet, J. L., Gibert, C., ... PNEUMA Trial Group. (2004). Impact of methicillin resistance on outcome of Staphylococcus aureus ventilator-associated pneumonia. American Journal of Respiratory Critical Care Medicine, 170(7), 786-792. 
Compton, F., Hoffmann, F., Hortig, T., Strauss, M., Frey, J., Zidek, W. \& Schäfer, J. H. (2008). Pressure ulcer predictors in ICU patients: Nursing skin assessment versus objective parameters. Journal of Wound Care, 17(10), 417-420, 422-424.

Cook, D. J., Griffith, L. E., Walter, S. D., Guyatt, G. H., Meade, M. O., Heyland, D. K., ... Canadian Critical Care Trials Group. (2001). The attributable mortality and length of intensive care unit stay of clinically important gastrointestinal bleeding in critically ill patients. Critical Care, 5(6), 368-375. doi:10.1186/cc1071

Cox, J. (2011). Predictors of pressure ulcers in adults critical care patients. American Journal of Critical Care, 20(5), 364-374. doi: 10.4037/ajcc2011934

Cramer, C. L., Orlowski , J. P., \& DeNicola, L. K. (2008). Pediatric intensivist extenders in the pediatric ICU. Pediatric Clinics of North America, 55(3), 687-708. doi: 10.1016/j.pcl.2008.02.007

Cravens, D.D. \& Zweig, S. (2000). Urinary catheter management. American Family Physician, 61(2), 369-376.

Daly, B. J., Douglas, S. L., Kelley, C. G., O'Toole, E. \& Montenegro, H. (2005). Trial of a disease management program to reduce hospital readmissions of the chronically critically ill. Chest, 128(2), 507-517. doi: 10.1378/chest.128.2.507

Dang, D., Johantgen, M. E., Pronovost, P. J., Jenckes, M. W., \& Bass, E. B. (2002). Postoperative complications: Does intensive care unit staff nursing make a difference? Heart Lung, 31(3), 219-228.

de Laat, E. H., Schoonhoven, L., Pickkers, P., Verbeek, A. L. \& van Achterberg, T. (2006). Epidemiology, risk and prevention of pressure ulcers in critically ill patients: A literature review. Journal of Wound Care, 15(6), 269-275.

Defloor, T., De Bacquer, D., \& Grypdonck, M. H. F. (2005). The effect of various combinations of turning and pressure reducing devices on the incidence of pressure ulcers. International Journal of Nursing Studies, 42(1), 37-46. doi: 10.1016/j.jinurstu.2004.05.013

Dellinger, R. P., Levy, M. M., Carlet, J. M., Bion, J., Parker, M. M., Jaeschke, R., ... International Surviving Sepsis Campaign Guidelines Committee. (2008). Surviving Sepsis Campaign: International guidelines for management of severe sepsis and septic shock: 2008. Critical Care Medicine, 36(1), 296-327. doi: 10.1097/01.CCM.0000298158.12101.41 
Dimick, J.B., Pronovost, P. J., Heitmiller, R. F., \& Lipsett, P.A. (2001). Intensive care unit physician staffing is associated with decreased length of stay, hospital cost, and complications after esophageal resection. Critical Care Medicine, 29(4), 753758.

Dodek, P., Keenan, S., Cook, D., Heyland, D., Jacka, M., Hand, L., ... Canadian Critical Care Society. (2004). Evidence-based clinical practice guideline for the prevention of ventilator-associated pneumonia. Annals of Internal Medicine, 141(4), 305-313.

Donabedian, A. (1978). The quality of medical care. Science, 200(4344), 856-864. doi: $10.1126 /$ science. 417400

Donabedian, A. (1988). The quality of care: How can it be assessed? JAMA, 260(12), 1743-1748. doi: 10.1001/jama.1988.03410120089033

Donabedian, A. (2005). Evaluating the quality of medical care. The Milbank Quarterly, 83(4), 691-729. doi: 10.1111/j.1468-0009.2005.00397.x

Drakulovic, M. B., Torres, A., Bauer, T. T., Nicolas, J. M., Nogue, S. \& Ferrer, M. (1999) Supine body position as a risk factor for nosocomial pneumonia in mechanically ventilated patients: a randomized trial. The Lancet, 354(9193), $1851-1858$.

Dungan, K. M., Braithwaite, S. S., \& Preiser, J.C. (2009). Stress hyperglycaemia. The Lancet, 373(9677), 1798-1807. doi:10.1016/S0140-6736(09)60553-5

Durbin, C. G. (2006). Team model: Advocating for the optimal method of care delivery in intensive care unit. Critical Care Medicine, 34(3), S12-S17. doi: 10.1097/01.ccm.0000199985.72497.d1

Eachempati, S. R., Hydo, L. J., Shou, J., \& Barie, P. S. (2009). Does de-escalation of antibiotic therapy for ventilator-associated pneumonia affect the likelihood of recurrent pneumonia or mortality in critically ill surgical patients? Journal of Trauma-Injury Infection and Critical Care, 66(5), 1343-1348. doi: 10.1097/TA.0b013e31819dca4e

Eachempati, S. R., Hydo, L. J., \& Barie, P. S. (2001). Factors influencing the development of decubitus ulcers in critically ill surgical patients. Critical Care Medicine, 29(9), 1678-1682.

Edwards, M. (1994). The rationale for the use of risk calculators in pressure sore prevention, and the evidence of the reliability and validity of published scales. Journal of Advanced Nursing, 20(2), 288-296. doi:10.1046/j.13652648.1994.20020288.x 
Edwards, J. R., Peterson, K. D., Mu, Y., Banerjee, S., Allen-Bridson, K., Morrell, G. ... Horan, T. C. (2009). National Healthcare Safety Network (NHSN) report: Data summary for 2006 through 2008, issued December 2009. American Journal of Infection Control, 37(10), 783-805. doi: 10.1016/j.ajic.2009.10.001

Elliott, R., McKinley, S., \& Fox, V. (2008). Quality improvement program to reduce the prevalence of pressure ulcers in an intensive care unit. American Journal of Critical Care, 17(4), 328-334.

Ely, E.W. (2000). The utility of weaning protocols to expedite liberation from mechanical ventilation. Respiratory Care Clinics of North America, 6(2), 303-19, vi.

Ely, E. W., Meade, M. O., Haponik, E. F., Kollef, M. H., Cook, D. J., Guyatt, G. H., \& Stoller, J. K. (2001). Mechanical ventilator weaning protocols driven by nonphysician health-care professionals: Evidence-based clinical practice guidelines. Chest, 120(Suppl. 6), 454S-463S. doi:

10.1378/chest.120.6_suppl.454S

Elpern, E. H., Killeen, K., Ketchum, A., Wiley, A., Patel, G., \& Lateef, O. (2009).

Reducing use of indwelling urinary catheters and associated urinary tract infections. American Journal of Critical Care, 18(6), 535-541. doi: 10.4037/ajcc2009938

Epstein, S. K., Ciubotaru, R. L., \& Wong, J. B. (1997). Effect of failed extubation on the outcome of mechanical ventilation. Chest, 112(1), 186-192. doi: 10.1378/chest.112.1.186

Epstein, S. K., Nevins, M. L., \& Chung, J. (2000). Effect of unplanned extubation on outcome of mechanical ventilation. American Journal of Respiratory and Critical Care Medicine, 161(6), 1912-1916.

Ewart, G. W., Marcus, L., Gaba, M. M., Bradner, R. H., Medina, J. L., \& Chandler, E. B. (2004). The critical care medicine crisis: A call for federal action: A white paper from the Critical Care Professional Societies. Chest, 125(4), 1518-1521.

Fakih, M. G., Dueweke, C., Meisner, S., Berriel-Cass, D., Savoy-Moore, R., Brach, N., ... Saravolatz, L. D. (2008). Effect of nurse-led multidisciplinary rounds on reducing unnecessary use of urinary catheterization in hospitalized patients. Infection Control and Hospital Epidemiology, 29(9), 815-819. doi: $10.1086 / 589584$

Finfer, S., Chittock, D. R., Su, S. Y., Blair, D., Foster, D., Dhingra, V., ... Robinson, B. (2009). Intensive versus conventional glucose control in critically ill patients. The New England Journal of Medicine, 360(13), 1283-1297. 
Finfer, S. \& Heritier, S. (2009). The NICE-SUGAR (Normoglycaemia in Intensive Care Evaluation and Survival Using Glucose Algorithm Regulation) study: Statistical analysis plan. Critical Care and Resuscitation, 11(1), 46-57.

Fink, J. B., Krause, S. A., Barrett, L., Schaaff, D., \& Alex, C. G. (1998). Extending ventilator circuit change interval beyond 2 days reduces the likelihood of ventilator-associated pneumonia. Chest, 113(2), 405-411. doi: 10.1378/chest.113.2.405

Finkler, S. A. \& Getzen, T. E. (2008). Costs and value. In Kovner, A. R. \& Knickman, J. R. (Eds.), Jonas \& Kovner's Health Care delivery in the United States (9th ed.) (pp. 479-506). New York: Springer Publishing Company.

Frantz, R. A., Gardner, S., Harvey, P., \& Specht, J. (1991). The cost of treating pressure ulcers in a long-term care facility. Decubitus, 4(3), 37-38, 40, 42.

Fraser, G. L., Riker, R. R., Prato, B.S., \& Wilkins, M. L. (2001). The frequency and cost of patient-initiated device removal in the ICU. Pharmacotherapy, 21(1), 1-6.

Frezza, E. E., Carleton, G. L., Valenziano, C. P. (2000). A quality improvement and risk management initiative for surgical ICU patients: A study of the effects of physical restraints and sedation on the incidence of self extubation. American Journal of Medical Quality, 15(5), 221-225.

Fuchs, R. J., Berenholtz, S. M., \& Dorman, T. (2005). Do intensivists in ICU improve outcome? Best Practice Research Clinical Anesthesiology, 19(1), 125-135.

Gajic, O., \& Afessa, B. (2009). Physician staffing models and patient safety in the ICU. Chest, 135(4), 1038-1044. doi: 10.1378/chest.08-1544

Gajic, O., Afessa, B., Hanson, A. C., Krpata, T., Yilmaz, M., Mohamed, S. F., ... Wylam, M. E. (2008). Effect of 24-hour mandatory versus on-demand critical care specialist presence on quality of care and family and provider satisfaction in the intensive care unit of a teaching hospital. Critical Care Medicine, 36(1), 36-44.

Girard, T. D., Kress, J. P., Fuchs, B. D., Thomason, J. W. W., Schweickert, W. D., Pun, B. T., ... Ely, E. W. (2008). Efficacy and safety of a paired sedation and ventilator weaning protocol for mechanically ventilated patients in intensive care (awakening and breathing controlled trial): A randomized controlled trial. The Lancet, 126(9607), 126-134. doi:10.1016/S0140-6736(08)60105-1

Giraud, T., Dhainaut, J. F., Vaxelaire, J. F., Joseph, T., Journois, D., Bleichner, G., ... Monsallier, J. F. (1993). Iatrogenic complications in adult intensive care units: A prospective two-center study. Critical Care Medicine, 21(1), 40-51. 
Goolsby, M. J. (2005). 2004 AANP National Nurse Practitioner Sample Survey, Part I: An overview. Journal of the American Academy of Nurse Practitioners, 17(9), 337-341. doi: 10.1111/j.1745-7599.2005.00060.x

Gordon, C. R., Axelrad, A., Alexander, J. B., Dellinger, R. P., \& Ross, S. E. (2006). Care of critically ill surgical patients using the 80-hour Accreditation Council of Graduate Medical Education Work-Week Guidelines: A survey of current strategies. The American Surgeon, 72(6), 497-499.

Gracias, V. H., Sicoutris, C. P., Stawicki, S. P, Meredith, D. M., Horan, A. D., Gupta, R., ... Schwab, C. W. (2008). Critical care nurse practitioners improve compliance with clinical practice guidelines in "semiclosed" surgical intensive care unit. Journal of Nursing Care Quality, 23(4), 338-344.

Grap, M. J.,Munro, C. L., Elswick, R. K., Jr., Sessler, C. N., \& Ward, K. R. (2004). Duration of action of a single, early oral application of chlorihexidine on oral microbial flora in mechanically ventilated patients: A pilot study. Heart Lung, 33(2), 83-91. doi: 10.1016/j.hrtlng.2003.12.004

Grap, M. J., Munro, C. L., Hummel, R. S., III., Elswick, R. K., Jr., McKinney, J. L., \& Sessler, C. N. (2005). Effect of backrest elevation on the development of ventilator-associated pneumonia. American Journal of Critical Care, 14(4), 325333.

Grap, M. J., Munro, C. L., Bryant, S., \& Ashtiani, B. (2003). Predictors of backrest elevation in critical care. Intensive and Critical Care Nursing, 19(2), 68-74. doi: 10.1016/S0964-3397(03)00028-4

Grenvik, A., \& Pinsky, M. R. (2009). Evolution of the intensive care unit as a clinical center and critical care medicine as a discipline. Critical Care Clinics, 25(1); 239250. doi: $10.1016 /$ j.ccc.2008.11.001

Grey, N. J., \& Perdrizet, G. A. (2004). Reduction of nosocomial infections in the surgical intensive care unit by strict glycemic control. Endocrine Practice, 10(Suppl. 2), 46-52.

Gruenberg, D. A., Shelton, W., Rose, S. L., Rutter, A. E., Socaris, S., \& McGee, G. (2006). Factors influencing length of stay in the intensive care unit. American Journal of Critical Care, 15(5), 502-509.

Guidelines Committee, Society of Critical Care Medicine. (1992). Guidelines for the definition of an intensivist and the practice of critical care medicine. Guidelines Committee; Society of Critical Care Medicine. Critical Care Medicine, 20(4), $540-542$. 
Halpern, N. A., \& Pastores, S. M. (2010). Critical care medicine in the United States 2000-2005: An analysis of bed numbers, occupancy rates, payer mix, and costs. Critical Care Medicine, 38(1), 65-71. doi: 10.1097/CCM.0b013e3181b090d0

Hanson, C. W., III., Deutschman, C. S., Anderson, H. L., III., Reilly, P. M., Behringer, E. C., Schwab, C. W., \& Price, J. (1999). Effects of an organized critical care service on outcomes and resource utilization: A cohort study. Critical Care Medicine, 27(2), 270-274.

Hanson, C. W., III., Durbin, C. G., Jr., Maccioli, G. A., Deutschman, C. S., Sladen, R. N., Pronovost, P. J., \& Gattioni, L. (2001). The anesthesiologist in critical care medicine: Past, present, and future. Anesthesiology, 95(3), 781-788.

Gould, C. V., Umscheid, C. A., Agarwal, R. K., Kuntz, G., Pegues, D. A., \& the Healthcare Infection Control Practices Advisory Committee (HICPAC). (2009). Guideline for prevention of catheter-associated urinary tract infections 2009. Retrieved from: http://www.cdc.gov/hicpac/pdf/CAUTI/CAUTIguideline2009final.pdf

Hermanides, J., Bosman, R. J., Vriesendorp, T. M., Dotsch, R., Rosendaal, F. R., Zandstra, D. F., ... DeVries, J. H. (2010). Hypoglycemia is associated with intensive care unit mortality. Critical Care Medicine, 38(6), 1430-1434. doi: 10.1097/CCM.0b013e3181de562c

Hoffman, L. A., Happ, M. B., Scharfenberg, C., DiVirgilio-Thomas, D., \& Tasota, F. J. (2004). Perceptions of physicians, nurses, and respiratory therapists about the role of acute care nurse practitioners. American Journal of Critical Care, 13(6), 480488.

Hoffman, L. A., Tasota, F. J., Zullo, T. G., Scharfenberg, C., \& Donahoe, M. P. (2005). Outcomes of care managed by an acute care nurse practitioner/attending physician team in a subacute medical intensive care unit. American Journal of Critical Care, 14(2), 121-130.

Horan, T. C., Andrus, M., \& Dudeck, M. A. (2008). CDC/NHSN surveillance definition of health care-associated infection and criteria for specific types of infections in the acute care setting. American Journal of Infection Control, 36(5), 309-332. doi: 10.1016/j.ajic.2008.03.002

Horrocks, S., Anderson, E., \& Salisbury, C. (2002). Systematic review of whether nurse practitioners working in primary care can provide equivalent care to doctors. $B M J, 324(7341), 819-823$. doi: 10.1136/bmj.324.7341.819 
Huang, W.-C., Wann, S.-R, Lin , S.-L, Kunin, C. M., Kung, M.-H, Lin , C.-H, .. Lin, T.-W. (2004). Catheter-associated urinary tract infections in intensive care units can be reduced by prompting physicians to remove unnecessary catheters. Infection Control and Hospital Epidemiology, 25(11), 974-978. doi: 10.1086/ 502329

Health and Human Services (HHS). (2009). HHS action plan to prevent healthcareassociated infections: Executive summary. Retrieved from: http://chfs.ky.gov/NR/rdonlyres/26602C0F-C49F-4E35-B3427FC4047C5C3B/0/HHSACTIONPLANTOPREVENTHAIs01062009.pdf

Institute for Healthcare Improvement (IHI). (2010). Implement the ventilator bundle. Retrieved from: http://www.ihi.org/IHI/Topics/CriticalCare/IntensiveCare/Changes/Implementthe VentilatorBundle.htm

Institute for Healthcare Improvement (IHI). (2011a). Establish a glycemic control policy in your ICU. Retrieved from:

http://www.ihi.org/knowledge/Pages/Changes/EstablishaGlycemicControlPolicyi nYourICU.aspx

Institute for Healthcare Improvement (IHI). (2011b). Implement Effective Glucose Control.

Retrieved from:

http://www.ihi.org/knowledge/Pages/Changes/ImplementEffectiveGlucoseContro 1.aspx

International Council of Nurses. (2008). Definitions and Characteristics of the Role. Retrieved from: http://www.icn-apnetwork.org

Irvine, D., Sidani, S., Porter, H., O’Brien-Pallas, L., Simpson, B., McGillis Hall, L., ... Nagel, L. (2000). Organizational factors influencing nurse practitioners' role implementation in acute care settings. Canadian Journal of Nursing Leadership, 13(3), 28-35.

Jahnigen, D. W., Baxter, C. R., \& Bodenbender, R. H. (1989). Pressure ulcers: Prevalence, cost and risk assessment: Consensus development conference statement. Decubitus; 2(2), 24-30.

Jarvis, W. R. (2007). The United States approach to strategies in the battle against healthcare-associated infections, 2006: transitioning from benchmarking to zero tolerance and clinician accountability. Journal of Hospital Infection, 65(Suppl. 2), 3-9. doi:10.1016/S0195-6701(07)60005-X 
Jegers, M., Edbrooke, D. L., Hibbert, C. L., Chalfin, D. B. \& Burchardi, H. (2002). Definitions and methods of cost assessment: An intensivist's guide. ESICM section on health research and outcome working group on cost effectiveness. Intensive Care Medicine, 28(6), 680-685. doi: 10.1007/s00134-002-1279-5

Jiricka, M. K., Ryan, P., Carvalho, M. A., \& Bukvich, J. (1995). Pressure ulcer risk factors in an ICU population. American Journal of Critical Care, 4(5), 361-367.

Kavanagh, B. P. \& McCowen, K. C. (2010). Glycemic control in the ICU. New England Journal of Medicine, 363(26), 2540-2546. doi:10.1056/NEJMcp1001115

Keller, P. B., Wille, J., van Ramshorst, B., van der Werken, C. (2002). Pressure ulcers in intensive care patients: A review of risks and prevention. Intensive Care Medicine, 28(10), 1379-1388. doi:10.1007/s00134-002-1487-z

Kieninger, A. N., \& Lipsett, P. A. (2009). Hospital-acquired pneumonia: Pathophysiology, diagnosis, and treatment. Surgical Clinics of North America, 89(2), 439-461. doi:10.1016/j.suc.2008.11.001

Kinnersley, P., Anderson, E., Parry, K., Clement, J., Archard, L, Turton, P., ... Rogers, C. (2000). Randomized controlled trial of nurse practitioner versus general practitioner care for patients requesting "same day" consultations in primary care. $B M J, 320$ (7241), 1043-1048. doi: 10.1136/bmj.320.7241.1043

Kleinpell, R. M. (1997). Acute-care nurse practitioners: Roles and practice profiles. AACN Clinical Issues, 8(1), 156-162.

Kleinpell, R. M. (1998). Reports of role descriptions of acute care nurse practitioners. AACN Clinical Issues, 9(2), 290-295.

Kleinpell, R. M. (1999). Evolving role descriptions of the acute care nurse practitioner. Critical Care Nursing Quarterly, 21(4), 9-15.

Kleinpell, R. M. (2005). Acute care nurse practitioner practice: Results of a 5-year longitudinal study. American Journal of Critical Care, 14(3), 211-219.

Kleinpell, R. \& Gawlinski, A. (2005). Assessing outcomes in advance practice nursing practice: The use of quality indicators and evidence-based practice. $A A C N$ Clinical Issues, 16(1), 43-57.

Kleinpell, R., \& Goolsby, M. J. (2006). 2004 American Academy of Nurse Practitioners National Nurse Practitioner Sample Survey: Focus on acute care. Journal of the American Academy of Nurse Practitioners, 18(9), 393-394. doi: 10.1111/j.17457599.2006.00154.x 
Kleinpell, R. M., Hravnak, M., Werner, K. E., \& Guzman, A. (2006). Skills taught in acute care NP programs: A national survey. Nurse Practitioner, 31(2), 7, 11-13.

Klevens, R. M., Edwards, J. R., Richards, C. L., Jr., Horan, T. C., Gaynes, R. P., Pollock, D. A., \& Cardo, D. M. (2007). Estimating health care-associated infections and deaths in U.S. hospitals, 2002. Public Health Reports, 122(2), 160-166.

Knaus, W. A., Draper, E. A., Wagner, D. P., \& Zimmerman, J. E. (1985). APACHE II: A severity of disease classification system. Critical Care Medicine, 13(10), 818829.

Knaus, V.L., Felten, S., Burton, S., Forbes, P., \& Davis, K. (1997). The use of nurse practitioners in the acute care setting. Journal of Nursing Administration, 27(2) $20-27$.

Koenig, S. M. \& Truwit, J. D. (2006). Ventilator-associated pneumonia: Diagnosis, treatment, and prevention. Clinical Microbiology Reviews, 19(4), 637-657. doi:10.1128/CMR.00051-05

Kollef, M. H., Afessa, B., Anzueto, A., Veremakis, C., Kerr, K. M., Margolis, B. D., ... Schinner, R. (2008) Silver-coated endotracheal tubes and incidence of ventilatorassociated pneumonia: The NASCENT randomized trial. JAMA, 300(7), 805-813. doi: $10.1001 /$ jama.300.7.805

Kramer, A. A., \& Zimmerman, J. E. (2010). A predictive model for the early identification of patients at risk for prolonged intensive care unit length of stay. BMC Medical Informatics and Decision Making, 10, 27-36. doi:10.1186/14726947-10-27

Kress, J. P., \& Hall, J. B. (2006). Sedation in the mechanically ventilated patient. Critical Care Medicine, 34(10), 2541-2546.

Kress, J. P., Pohlman, A. S., O’Connor, M. F., \& Hall, J. B. (2000). Daily interruption of sedative infusions in critically ill patients undergoing mechanical ventilation. $\mathrm{New}$ England Journal of Medicine, 342(20), 1471-1477.

Krinsley, J. S. (2008). Glycemic variability: A strong independent predictor of mortality in critically ill patients. Critical Care Medicine, 36(11), 3008-3013. doi:10.1097/CCM.0b013e31818b38d2

Krinsley, J. S., \& Jones, R. L. (2006). Cost analysis of intensive glycemic control in critically ill adult patients. Chest, $129(3), 644-650$. doi:10.1378/chest.129.3.644 
Kuper, K. M. \& Septimus, E. J. (2009). Health-care-associated infections: The legislative perspective and the pharmacist's role. American Journal of Health-System Pharmacy, 66(5), 488-494.

Lahmann, N. A., Kottner, J., Dassen, T., \& Tannen, A. (2012). Higher pressure ulcer risk on intensive care?: Comparison between general wards and intensive care units. Journal of Clinical Nursing, 21(3-4), 354-361. doi:10.1111/j.13652702.2010.03550.x

Laupland, K. B., Bagshaw, S. M., Gregaon, D. B., Kirkpatrick, A. W., Ross, T., \& Church, D. L. (2005). Intensive care unit-acquired urinary tract infections in a regional critical care system. Critical Care, 9(2), R60-R65. doi: 10.1186/cc3023

Laurant, M., Reeves, D., Hermens, R., Braspenning, J., Grol, R., \& Sibbald, B. (2005). Substitution of doctors by nurses in primary care. The Cochrane Database of Systematic Reviews, July 2005, Issue 3. John Wiley \& Sons. Retrieved from www.cochrane.org/reviews/en/ab001271.html

Lembitz, A. (2010, August). Never say "never" events. Retrieved from http://www.aaos.org/news/aaosnow/aug10/managing5.asp

Leone, M., Garnier, F., Dubuc, M., Bimar, M. C., \& Martin, C. (2001). Prevention of nosocomial urinary tract infection in ICU patients: Comparison of effectiveness of two urinary drainage systems. Chest, 120(1), 220-224. doi: 10.1378/chest.120.1.220

Levinson, D. R. (2010). Adverse events in hospitals: National incidence among Medicare beneficiaries. Department of Health and Human Services Office of Inspector General, OEI-06-09-00090.

Levy, M.M., Rapoport, J., Lemeshow, S., Chalfin, D. B., Phillips, G., \& Danis, M. (2008). Association between critical care physician management and patient mortality in the intensive care unit. Annals of Internal Medicine, 148(11), 801809.

Lichtig, L. K., Knauf, R.A. \& Milholland, D. K. (1999). Some impacts of nursing on acute care hospital outcomes. Journal of Nursing Administration, 29(2): 25-33.

Lo, E., Nicolle, L., Classen, D., Arias, K. M., Podgorny, K., Anderson, D. J. ... Yokoe, D. S. (2008). Strategies to prevent catheter-associated urinary tract infections in acute care hospitals. Infection Control and Hospital Epidemiology, 29(Suppl. 1), S41-S50. doi: 10.1086/591066 
Lorente, L., Huidobro, M. S., Martin, M. M., Jimenez, A., \& Mora, M. L. (2004). Accidental catheter removal in critically ill patients: A prospective and observational study. Critical Care, 8(4), R229-R233. doi:10.1186/cc2874

Lucado, J., Paez, K., Andrews, R., \& Steiner, C. (2010). Adult hospital stays with infections due to medical care, 2007 (Statistical Brief No. 94). Retrieved from http://www.hcup-us.ahrq.gov/reports/statbriefs/sb94.pdf

MacLaren, R. \& Sullivan, P. W. (2006). Economic evaluation of sustained sedation/analgesia in the intensive care unit. Expert Opinion on Pharmacotherapy, 7(15), 2047-2068. doi:10.1517/14656566.7.15.2047

Maki, D. G. \& Tambyah, P. A. (2001). Engineering out the risk for infection with urinary catheters. Emerging Infectious Diseases, 7(2), 342-347.

Marik, P. E., \& Preiser, J.-C. (2010). Toward understanding tight glycemic control in the ICU: A systemic review and metaanalysis. Chest, 137(3), 544-551. doi:10.1378/chest.09-1737

Mascia, M. F., Koch, M., Medicis, J. J., (2000). Pharmacoeconomic impact of rational use guidelines on the provision of analgesia, sedation, and neuromuscular blockade in critical care. Critical Care Medicine, 28(7), 2300-2306.

McGillis Hall, L., Doran, D., \& Pink, G. H. (2004). Nurse staffing models, nursing hours, and patient safety outcomes. Journal of Nursing Administration, 34(1), 41-45.

McLaughlin, R. (2007). Preparation for negotiating scope of practice for acute care nurse practitioners. Journal of the American Academy of Nurse Practitioners, 19(12), 627-634.

McMullen, M., Alexander, M. K., Bourgeois, A., \& Goodman, L. (2001). Evaluating a nurse practitioner service. Dimensions of Critical Care Nursing, 20(5), 30-34.

Melander, S., Kleinpell, R., \& McLaughlin, R. (2007). Ensuring clinical competency for NPs in acute care. The Nurse Practitioner, 32(4), 19-20.

Metheny, N. A., Clouse, R. E., Chang, Y.-H., Stewart, B. J., Oliver, D. A., \& Kollef, M. H. (2006). Tracheobronchial aspiration of gastric contents in critically ill tube-fed patients: Frequency, outcomes, and risk factors. Critical Care Medicine, 34(4), 1007-1015. doi: 10.1097/01.CCM.0000206106.65220.59

Meyer, S. C., \& Miers, L. J. (2005). Cardiovascular surgeon and acute care nurse practitioner: Collaboration on postoperative outcomes. AACN Clinical Issues, 16(2), 149-158. 
Mick, D. J., \& Ackerman, M. H. (2000). Advanced practice nursing role delineation in acute and critical care: Application of the Strong Model of Advanced Practice. Heart Lung, 29(3), 210-221.

Mion, L. C., Fogel, J., Sandhu, S., Palmer, R. M., Minnick, A. F., Cranston, T., ... \& Leipzig, R. (2001). Outcomes following physical restraint reduction programs in two acute care hospitals. The Joint Commission Journal on Quality Improvement, 27(11), 605-618.

Mion, L. C., Minnick, A. F., Leipzig, R. M., Catrambone, C. D., \& Johnson, M. E. (2007). Patient-initiated device removal in intensive care units: A national prevalence study. Critical Care Medicine, 35(12), 2714-2720. doi:10.1097/01.CCM.0000291651.12767.52

Mitchell-DiCenso, A., Guyatt, G., Marrin, M., Goeree, R., Willan, A., Southwell, D., ... Baumann, A. (1996). A controlled trial of nurse practitioners in neonatal intensive care. Pediatrics, 98(6), 1143-1148.

Mitchell, C. C., Ashley, S. W., Zinner, M. J., \& Moore, F. D., Jr. (2007). Predicting future staffing needs at teaching hospitals. Archives of Surgery, 142(4), 329-334.

Moons, P., Sels, K., De Becker, W., De Guest, S., \& Ferdinande, P. (2004). Development of a risk assessment tool for deliberate self-extubation in intensive care patients. Intensive Care Medicine, 30(7), 1348-1355.

Mundinger, M. O. (1999). Can advanced practice nurses succeed in the primary care market? Nursing Economics, 17(1), 7-14.

Mundinger, M. O., Kane, R. L., Lenz, E. R., Totten, A. M., Tsai, W.-Y., Cleary, P. D., ... Shelanski, M. L. (2000). Primary care outcomes in patients treated by nurse practitioners or physicians: A randomized trial. JAMA, 283(1), 59-68. doi: 10.1001/jama.283.1.59

Munro, C. L., Grap, M. J., Jones, D. J., McClish, D. K., \& Sessler, C. N. (2009). Chlorhexidine, toothbrushing, and preventing ventilator-associated pneumonia in critically ill adults. American Journal of Critical Care, 18(5), 428-437. doi: 10.4037/ajcc2009792

Munro, N. \& Taylor-Panek, S. (2007). The nurse practitioner role: The communication link for cardiac surgery patients. Critical Care Nursing Clinics of North America, 19(4), 385-394. doi: 10.1016/j.ccell.2007.08.002 
Nathens, A. B., Rivara, F. P., MacKenzie, E. J., Maier, R. V., Wang, J., Egleston, B., ...Jurkovich, G. J. (2006). The impact of an intensivist-model ICU on traumarelated mortality. Annals of Surgery, 244(4), 545-554. doi: 10.1097/01.sla.0000239005.26353.49

National Association of Clinical Nurse Specialist (NACS) (n.d.). Frequently asked questions. Retrieved from http://nacns.org/AboutNACNS/FAQs/tabid/109/Default.aspx

National Healing Corporation. (2005a). Pressure ulcer, wound healing perspectives. National Healing Corporation, 2(1).

National Healing Corporation. (2005b). Interventions in managing pressure ulcers. Wound Healing Perspectives, 2(1), 1-8.

Naylor, M. D., Brooten, D., Campbell, R., Jacobsen, B. S., Mezey, M. D., Pauley, M. V., \& Schwartz, J. S. (1999). Comprehensive discharge planning and home follow-up of hospitalized elders: A randomized controlled trial. JAMA, 281(7), 613-620. doi: 10.1001/jama.281.7.613

Naylor, M. D., Brooten, D. A., Campbell, R. L., Maislin, G., McCauley, K. M., \& Schwartz, J. S. (2004). Transitional care of older adults hospitalized with heart failure: A randomized, controlled trial. Journal of the American Geriatrics Society, 52(5), 675-684.

Needleman, J., Buerhaus, P., Mattke, S., Stewart, M., \& Zelevinsky, K. (2002a). Nursestaffing levels and the quality of care in hospitals. New England Journal of Medicine, 346(22), 1715-1722.

Needleman, J., Buerhaus, P., Mattke, S., Stewart, M., \& Zelevinsky, K. (2002b) Nurse staffing and quality of care in hospitals in the United States. Policy, Politics, \& Nursing Practice, 3(4), 306-308.

Page, N. E. \& Arena, D. M. (1994). Rethinking the merger of the clinical nurse specialist and the nurse practitioner roles. Image: Journal of Nursing Scholarship, 26(4), 315-318.

Parekh, A. (2008). HHS efforts to reduce healthcare-associated infections [PowerPoint Slides]. Retrieved from http://www.cdc.gov/hicpac/pdf/HHSpresentationHICPAC_11_08.pdf

Pender, L. R., \& Frazier, S. K. (2005). The relationship between dermal pressure ulcers, oxygenation and perfusion in mechanically ventilated patients. Intensive and Critical Care Nursing 21(1), 29-38. doi:10.1016/j.iccn.2004.07.005 
Person, S. D., Allison, J. J., Kiefe, C. I., Weaver, M. T., Williams, O. D., Centor, R. M., \& Weissman, N. W. (2004). Nurse staffing and mortality for Medicare patients with acute myocardial infarction. Medical Care, 42(1), 4-12. doi: 10.1097/01.mlr.0000102369.67404.b0

Pioro, M. H., Landefeld, C. S., Brennan, P. F., Daly, B., Fortinsky, R. H., Kim, U., \& Rosenthal, G. E. (2001). Outcomes-based trial of an inpatient nurse practitioner service for general medical patients. Journal of Evaluation in Clinical Practice, $7(1), 21-33$.

Pokorny, M. E., Koldjeski, D., \& Swanson, M. (2003). Skin care intervention for patients having cardiac surgery. American Journal of Critical Care, 12(6), 535-544.

Polderman, K. H., Girbes, A. R., Thijs, L. G., \& Strack van Schijndel, R. J. M. (2001). Accuracy and reliability of APACHE II scoring in two intensive care units Problems and pitfalls in the use of APACHE II and suggestions for improvement. Anaesthesia, 56(1), 47-50.

Polderman, K. H., Jorna, E. M., \& Girbes, A. R. (2001). Inter-observer variability in APACHE II scoring: Effect of strict guidelines and training. Intensive Care Medicine, 27(8), 1365-1369. doi: 10.1007/s001340101012

Polderman, K. H., Thijs, L. G., \& Girbes, A. R. (1999). Interobserver variability in the use of APACHE II scores. The Lancet, 353(9150), 380. doi:10.1016/S01406736(05)74953-9

Pollack, M. M., Katz, R. W., Ruttimann, U. E., \& Getson, P. R. (1988). Improving the outcome and efficiency of intensive care: The impact of an intensivist. Critical Care Medicine, 16(1), 11-17.

Pronovost, P. J., Angus, D. C., Dorman, T., Robinson, K. A., Dremsizov, T. T., \& Young, T. L. (2002). Physician staffing patterns and clinical outcomes in critically ill patients: A systematic review. JAMA, 288(17), 2151-2162.

Pronovost, P. J., Dang, E., Dorman, T., Lipsett, P. A., Garrett, E., Jenckes, M., Bass, E. B. (2001). Intensive care unit nurse staffing and the risk for complications after abdominal aortic surgery. Effective Clinical Practice, 4(5), 199-206.

Pronovost, P. J., Jenckes, M. W., Dorman, T., Garret, E., Breslow, M. J, Rosenfeld, B. A., ... Bass, E. (1999). Organizational characteristics of intensive care units related to outcomes of abdominal aortic surgery. JAMA, 281(14), 1310-1317. doi: 10.1001/jama.281.14.1310 
Pronovost, P. J., Young, T. L., Dorman, T., Robinson, K., \& Angus, D. C. (1999). Association between ICU physician staffing and outcomes: A systematic review. Critical Care Medicine, 27(12) S, A43.

Pyenson, B., Murphy-Barron, C. M., Mirkin, D. \& Iwasaki, K. (2007). Economic impact of hospital-acquired infections on hospitals. Retrieved from http://publications.milliman.com/research/health-rr/pdfs/economic-impact-ofhospital-10-17-07.pdf

Rady, M. Y., \& Ryan, T. (1999). Perioperative predictors of extubation failure and the effect on clinical outcome after cardiac surgery. Critical Care Medicine, 27(2), 340-347.

Raines, L. (2008). Acute care nurse practitioner at Emory on the rise. The AtlantaJournal Constitution. Retrieved from http://www.ajc.com/hotjobs/content/hotjobs/careercenter/pulse/2008/10/19/acute_ care_nurse.html

Reilly, E. F., Karakousis, G. C., Schrag, S. P., \& Stawicki, S. P. (2007). Pressure ulcers in the intensive care unit: The 'forgotten' enemy. OPUS 12 Scientist, 1(2), 17-30.

Rello, J., Ollendorf, D. A., Oster, G., Vera-Llonch, M., Bellm, L., Redman, R., \& Kollef, M. H. (2002). Epidemiology and outcomes of ventilator-associated pneumonia in a large US database. Chest, 122(6), 2115-2121.doi: 10.1378/chest.122.6.2115

Reynolds, H. N., Haupt, M. T., Thill-Bahazorian, M. C., \& Carlson, R. W. (1988). Impact of critical care physician staffing of patients with septic shock in a university hospital medical intensive care unit. JAMA, 260(23), 3446-3450. doi: 10.1001/jama.1988.03410230064029

Richman, P. S., Baram, D., Varela, M., \& Glass, P. S. (2006). Sedation during mechanical ventilation: A trial of benzodiazepine and opiate in combination. Critical Care Medicine, 34(5), 1395-1401. doi: 10.1097/01.CCM.0000215454.50964.F8

Rivers, K. (2010, September 9). VUSN, Anesthesia partner to expand ICU nurse training. VUMC Reporter. Retrieved from: http://www.mc.vanderbilt.edu/reporter/index.html?ID=9382

Rosenthal, V. D., Guzman, S., \& Safdar, N. (2004). Effect of education and performance feedback on rates of catheter-associated urinary tract infection in intensive care units in Argentina. Infection Control and Hospital Epidemiology, 25(1), 47-50. 
Rudy, E. B., Davidson, L. J., Daly, B., Clochesy, J. M., Sereika, S., Baldisseri, M., ... Ryan, C. (1998). Care activities and outcomes of patients cared for by acute care nurse practitioners, physician assistants, and resident physicians: A comparison. American Journal of Critical Care, 7(4), 267-281.

Russell, D., VorderBruegge, M., \& Burns, S. M. (2002). Effect of an outcomes-managed approach to care of neuroscience patients by acute care nurse practitioners. American Journal of Critical Care, 11(4), 353-362.

Russo, C. A., Steiner, C., \& Spector, W. (2008). Hospitalizations related to pressure ulcers among adults 18 years and older, 2006 (Statistical Brief No. 64). Retrieved from http://www.hcup-us.ahrq.gov/reports/statbriefs/sb64.pdf

Safdar, N. \& Abad, C. (2008). Educational interventions for prevention of healthcareassociated infection: A systematic review. Critical Care Medicine, 36(3), 933940. doi: 10.1097/CCM.0B013E318165FAF3

Safdar, N., Dezfulian, C., Collard, H. R., \& Saint, S. (2005). Clinical and economic consequences of ventilator-associated pneumonia: A systematic review. Critical Care Medicine, 33(10), 2184-2193.

Schoonhoven, L., Defloor, T., \& Grypdonck, M. H. F. (2002). Incidence of pressure ulcers due to surgery. Journal of Clinical Nursing, 11(4), 479-487.

Schweickert, W. D., Gehlbach, B. K., Pohlman, A. S., Hall, J. B., \& Kress, J. P. (2004). Daily interruptions of sedative infusions and complications of critical illness in mechanically ventilated patients. Critical Care Medicine, 32(6), 1272-1276. doi:10.1097/01.CCM.0000127263.54807.79

Scott, R.D., II. (2009). The direct medical costs of healthcare-associated infections in U.S. hospitals and the benefits of prevention. Retrieved from: http://www.cdc.gov/hai/pdfs/hai/scott_costpaper.pdf

Segers, P., Speekenbrink, R. G. H., Ubbink, D. T., van Ogtrop, M. L., \& de Mol, B. A. (2006). Prevention of nosocomial infection in cardiac surgery by decontamination of the nasopharynx and oropharynx with chlorhexidine gluconate: A randomized control trial. JAMA, 296(20), 2460-2466. doi: 10.1001/jama.296.20.2460

Sessler, C. N., Grap, M. J., Brophy, G. M. (2001). Multidisciplinary management of sedation and analgesia in critical care. Seminars in Respiratory and Critical Care Medicine, 22(2), 211-226.

Sessler, C. N., Grap, M. J., Ramsay, M. A. E. (2008). Evaluating and monitoring analgesia and sedation in the intensive care unit. Critical Care, 12(Suppl. 3), S2. doi:10.1186/cc6148 
Shahin, E. S. M., Dassen, T., \& Halfens, R. J. G. (2008). Pressure ulcer prevalence and incidence in intensive care patients: A literature review. Nursing in Critical Care, 13(2), 71-79.

Shahin, E. S. M., Dassen, T., \& Halfens, R. J. G. (2009). Pressure ulcer prevention in intensive care patients: Guidelines and practice. Journal of Evaluation in Clinical Practice, 15(2), 370-374. doi:10.1111/j.1365-2753.2008.01018.x

Sheer, B., \& Wong, F. K. Y. (2008). The development of advanced nursing practice globally. Journal of Nursing Scholarship, 40(3), 204-211.

Shorr, A. F., Combes, A., Kollef, M. H., \& Chastre, J. (2006). Methicillin-resistant Staphylococcus aureus prolongs intensive care unit stay in ventilator-associated pneumonia despite initially appropriate antibiotic therapy. Critical Care Medicine, 34(3), 700-706.

Sidani, S. (2008). Effects of patient-centered care on patient outcomes: An evaluation. Research and Theory for Nursing Practice: An International Journal, 22(1), 2437.

Sidani, S., Doran, D., Porter, H., LeFort, S., O'Brien-Pallas, L. L., Zahn, C., \& Sarkissian, S. (2006). Outcomes of nurse practitioners in acute care: An exploration. The Internet Journal of Advanced Nursing Practice, 8(1). Retrieved from http://ispub.com/IJANP/8/1/12232

Smith, M. W., Barnett, P. G., Phibbs, C. S., \& Wagner, T. H. (2010). Microcost methods for determining $V A$ healthcare costs. Retrieved from Health Economics Resource Center website: http://www.herc.research.va.gov/files/BOOK_458.pdf

Society of Critical Care Medicine (SCCM). (2006). Critical care statistics in the United States. Retrieved from http://sccmwww.sccm.org/Documents/WebStatiticsPamphletFinalJune06.pdf

Sole, M. L., Hunkar-Huie, A. M., Schiller, J. S., \& Cheatham, M. L. (2001). Comprehensive trauma patient care by nonphysician providers. AACN Clinical Issues: AACN Clinical Issues, 12(3), 438-446.

Sole, M. L., Poalillo, F. E., Byers, J. F., \& Ludy, J. E. (2002). Bacterial growth in secretions and on suctioning equipment of orally intubated patients: A pilot study. American Journal of Critical Care, 11(2), 141-149.

Spisso, J., O’Callaghan, C., McKennan, M., \& Holcroft, J. W. (1990). Improved quality of care and reduction of housestaff workload using trauma nurse practitioners. The Journal of Trauma, 30(6), 660-663, 663-665. 
Starck, P. L. (2005). The cost of doing business in nursing education. Journal of Professional Nursing, 21(3), 83-190.

Stark, R. P., \& Maki, D. G. (1984). Bacteriuria in the catheterized patient. What quantitative level of bacteriuria is relevant? The New England Journal of Medicine, 311(9), 560-564.

Stickler, D. J., \& Morgan, S. D. (2008). Observations on the development of the crystalline bacterial biofilms that encrust and block Foley catheters. Journal of Hospital Infection, 69(4), 350-360. doi:10.1016/j.jhin.2008.04.031

Surviving Sepsis Campaign (SSC). (2005). The surviving sepsis campaign. Retrieved from: http://www.survivingsepsis.org/About_the_Campaign/Pages/AbouttheCampaign. aspx

Tambyah, P. A., Knasinski, V., \& Maki, D. G. (2002). The direct costs of nosocomial catheter-associated urinary tract infections in the era of managed care. Infection Control and Hospital Epidemiology, 23(1), 27-31.

Terekeci, H., Kucukardali, Y., Top, C., Onem, Y., Celik, S., \& Oktenli, C. (2009). Risk assessment study of the pressure ulcers in intensive care unit patients. European Journal of Internal Medicine, 20(4), 394-397. doi: 10.1016/j.ejim.2008.11.001

The Joint Commission. (2012, August 15). Surgical care improvement project. The Joint Commission on Accreditation of Healthcare Organizations (JCAHO). Retrieved from http://www.jointcommission.org/surgical_care_improvement_project/

The LeapFrog Group. (2008). LeapFrog Hospital Survey Results 2008. Retrieved from http://www.leapfroggroup.org/media/file/leapfrogreportfinal.pdf

Treggiari, M. M., Martin, D. P., Yanez, N. D., Caldwell, E., Hudson, L. D., \& Rubenfeld, G. D. (2007). Effect of intensive care unit organizational model and structure on outcomes in patients with acute lung injury. American Journal of Respiratory and Critical Care Medicine, 176(7), 685-690. doi: 10.1164/rccm.200701-165OC

U.S. Department of Health \& Human Services, Health Resources \& Services Administration. (2006). Report to Congress The critical care workforce: A study of the supply and demand for critical care physicians (Senate Report 108-81, 109103, and House Report 109-143). Washington, DC: U.S. Government Printing Office. 
U.S. Department of Health \& Human Services, Health Resources \& Services Administration. (2010). The registered nurse population: Findings from the 2008 National Sample Survey of Registered Nurses. Retrieved from http://bhpr.hrsa.gov/healthworkforce/rnsurvey2008.html

Valles, J., Artigas, A., Rello, J., Bonsoms, N., Fontanals, D., Blanch, L., ... Mestre, J. (1995). Continuous aspiration of subglottic secretions in preventing ventilatorassociated pneumonia. Annals of Internal Medicine, 122(3), 179-186

Vanderbilt University School of Nursing. (2007). Acute Care Nurse PractitionerIntensivist focus. Vanderbilt University. Retrieved from http://www.nursing.vanderbilt.edu/msn/acnp_intensivist.html

Van den Berghe, G., Wouters, P., Weekers, F., Verwaest, C., Bruyninckx, F., Schetz, M., ... \& Bouillon, R. (2001). Intensive insulin therapy in critically ill patients. The New England Journal of Medicine, 345(19), 1359-1367.

van Nieuwenhoven, C. A., Vandenbroucke-Grauls, C., van Tiel, F. H., Joore, H. C., van Schijndel, R. J., van der Tweel, I, ... Bonten, M. J. (2006). Feasibility and effects of the semirecumbent position to prevent ventilator-associated pneumonia: A randomized study. Critical Care Medicine, 34(2), 396-402.

VanGilder, C., Amlung, S., Harrison, P., \& Meyer, S. (2009). Results of the 2008-2009 International Pressure Ulcer Prevalence ${ }^{\mathrm{TM}}$ Survey and a 3-year, acute care, unitspecific analysis. Ostomy Wound Management; 55(11), 39-45.

Vazirani, S., Hays, R. D., Shapiro, M. F., \& Cowan, M. (2005). Effect of a multidisciplinary intervention on communication and collaboration among physicians and nurses. American Journal of Critical Care. 14(1) 71-77.

Warren, D. K., Shukla, S. J., Olsen, M. A., Kollef, M. H., Hollenbeak, C. S., Cox, M. J., ... Fraser, V. J. (2003). Outcome and attributable cost of ventilator associated pneumonia among intensive care unit patients in a suburban medical center. Critical Care Medicine, 31(5), 1312-1317. doi: 10.1097/01.CCM.0000063087.93157.06

Williams, T. A., Ho, K. M., Dobb, G. J., Finn, J. C., Knuiman, M., Webb, S. A. R., \& Royal Perth Hospital ICU Date Linkage Group. (2010). Effect of length of stay in intensive care unit on hospital and long-term mortality of critically ill adult patients. British Journal of Anaesthesia, 104(4), 459-464. doi:10.1093/bja/aeq025 
Wise, K. R., Akopov, V. A., Williams, B. R., Jr., Ido, M. S., Leeper, K. V., Jr., \& Dressler, D. D. (2011). Hospitalists and intensivists in the medical ICU: A prospective observational study comparing mortality and length of stay between two staffing models. Journal of Hospital Medicine, 7(3), 183-189. doi: 10.1002/jhm.972

Wyckoff, M., \& Kaliff, J. (2009). Acute care nurse practitioners: The intensivist theory [Abstract]. Virginia Henderson International Library. Retrieved from http://www.nursinglibrary.org/Portal/main.aspx?pageid=4024\&pid=21540

Yokoe, D. S., \& Classen, D. (2008). Improving patient safety through infection control: A new healthcare imperative. Infection Control and Hospital Epidemiology, 29(Suppl. 1), S3-S11.

Young, M. P., \& Birkmeyer, J. D. (2000). Potential reduction in mortality rates using an intensivist model to manage intensive care units. Effective Clinical Practice, 3(6), 284-289.

Zell, B. L., \& Goldmann, D, A. (2007). Healthcare-associated infection and antimicrobial resistance: Moving beyond description to prevention. Infection Control and Hospital Epidemiology, 28(3), 261-264. doi: 10.1086/513722 
VITA

\section{FRANCISCO GUIDO-SANZ}

Born, Holguin, Cuba

1994-1995

B.S.N., Nursing

Barry University

Miami Shores, Florida

2002

Certificate in Family-Focused Interdisciplinary Healthcare Across Cultures

Florida International University, Miami, Florida

University of Rome La Sapienza, Rome, Italy

$2001-2003$

M.S.N., Adult Health Nurse Practitioner

Florida International University

Miami, Florida

$2007-2008$

Post Master's Certificate in Nursing Education

Florida International University

Miami, Florida

2008-2014

Doctoral Candidate

Florida International University

Miami, Florida

\section{PUBLICATIONS AND PRESENTATIONS}

Brooten, D., Youngblut, J. M., Hannan, J., Guido-Sanz, F., Felber, D. \& Deosires, W. (2012). Health problems and APN interventions in pregnant women with diabetes.

Pacific Rim International Journal of Nursing Research, 16(2), 85 - 96.

Brooten, D., Youngblut, J. M., Deosires, W., Singhala, K., \& Guido-Sanz, F. (2011). Global considerations in measuring effectiveness of advanced practice nurses. International Journal of Nursing Studies. Retrieved from http://dx.doi.org/10.1016/j.ijnurstu.2011.10.022

Brooten, D., Youngblut, J. M., Hannan, J., \& Guido-Sanz, F. (2011). The impact of interprofessional collaboration on the effectiveness, significance, and future of Advanced Practice Nurses. Nursing Clinics of North America, 47(2), 283 - 294. doi:

10.1016/j.cnur.2012.02.005 
Gonzalez, J. E., Groom, J. A., Green, M., Guido-Sanz, F., Brown, M., \& Stage, S. et al. (2008). The effects of a simulation-based educational intervention on ACLS knowledge [Abstract]. American Association of Nurse Anesthetists Journal, 75(6), 376.

Guido-Sanz, F. (2011). Outcomes of APN-driven Initiatives on Healthcare Associated Infections in a Surgical Intensive Care Unit (SICU). [Podium presentation]. 6th International Conference on Health Issues in Arab Communities, Aleppo, Syria.

Brooten, D., Youngblut, J. M., Hannan, J., Guido-Sanz, F. \& Felber Neff, D. (2011). Pregnant women with diabetes: Health problems, APN interventions in 2 models of APN transitional care. [Abstract]. Presented at Sigma Theta Tau International Honor Society of Nursing 41st Biennial Convention 29 October - 2 November 2011, Grapevine, TX.

Brooten, D., Youngblut, J. M., Hannan, J., \& Guido-Sanz, F. (2011). Pregnant women with diabetes: Health problems, APN interventions in 2 models of APN transitional care. [Poster]. Presented at the 25th Annual Conference of the Southern Nursing Research Society, February, Jacksonville, FL.

Brooten, D., Youngblut, J. M., Hannan, J., \& Guido-Sanz, F. (2010). Pregnant women with diabetes: Health problems, APN interventions in 2 models of APN transitional care. [Paper]. Presented at the 4th Annual South Florida Research Consortium: Best Evidence from Research: The Gateway to Excellent Care, October, Miami, FL.

Guido-Sanz, F. \& Hernandez, S. (2010). Outcomes of APN-driven Initiatives on Healthcare Associated Infections in a Surgical Intensive Care Unit (SICU). [Podium presentation]. Research Consortium of Nursing Consortium of South Florida, Parrot Jungle Island, Miami, FL.

Archer, A. \& Guido-Sanz, F. (2009). PUPPIEs (Pressure Ulcer Prevention, Prevalence, and Incidence Evaluation) in the SICU: An APN initiative. [Podium presentation]. Research Consortium of Nursing Consortium of South Florida, Florida International University, Miami, FL. 